\title{
Sexual risk behavior and mental health of young gay men
}

Citation for published version (APA):

Franssens, D. (2010). Sexual risk behavior and mental health of young gay men. [Doctoral Thesis, Maastricht University]. Datawyse / Universitaire Pers Maastricht. https://doi.org/10.26481/dis.20100115df

Document status and date:

Published: 01/01/2010

DOI:

$10.26481 /$ dis.20100115df

Document Version:

Publisher's PDF, also known as Version of record

\section{Please check the document version of this publication:}

- A submitted manuscript is the version of the article upon submission and before peer-review. There can be important differences between the submitted version and the official published version of record.

People interested in the research are advised to contact the author for the final version of the publication, or visit the DOI to the publisher's website.

- The final author version and the galley proof are versions of the publication after peer review.

- The final published version features the final layout of the paper including the volume, issue and page numbers.

Link to publication

\footnotetext{
General rights rights.

- You may freely distribute the URL identifying the publication in the public portal. please follow below link for the End User Agreement:

www.umlib.nl/taverne-license

Take down policy

If you believe that this document breaches copyright please contact us at:

repository@maastrichtuniversity.nl

providing details and we will investigate your claim.
}

Copyright and moral rights for the publications made accessible in the public portal are retained by the authors and/or other copyright owners and it is a condition of accessing publications that users recognise and abide by the legal requirements associated with these

- Users may download and print one copy of any publication from the public portal for the purpose of private study or research.

- You may not further distribute the material or use it for any profit-making activity or commercial gain

If the publication is distributed under the terms of Article $25 \mathrm{fa}$ of the Dutch Copyright Act, indicated by the "Taverne" license above, 
Sexual risk behavior and mental health of young gay men 


\section{Colophon}

Graphic design: Hugo Alberts

ISBN: 9789052789033

Production: Datawyse I Universitaire Press Maastricht

(C) 2009 Dirk Franssens, Maastricht

The research presented in this dissertation was supported by a grant of the AidsFonds, the Netherlands (grant number 2005050).

All rights are reserved. No part of this book may be reproduced or transmitted in any form or by any means, without written permission from the author or, when appropriate, the publisher of the article. 


\section{Sexual risk behavior and mental health of young gay men}

\section{PROEFSCHRIFT}

Ter verkrijging van de graad van doctor aan de Universiteit Maastricht, op gezag van de Rector Magnificus, Prof. mr. G. P. M. F. Mols, volgens het besluit van het College van Decanen, in het openbaar te verdedigen op vrijdag 15 januari 2010 om 14:00 uur.

door

Dirk Franssens

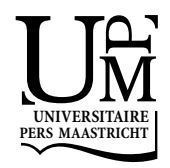




\section{Promotores}

Prof. dr. Harm J. Hospers

Prof. dr. Gerjo Kok

\section{Beoordelingscommissie}

Prof. dr. Harald Merckelbach (voorzitter)

Prof. dr. Charles Abraham (University of Sussex, Brighton \& UM)

Dr. Arjan Bos (UM)

Dr. Udi Davidovich (GGD Amsterdam)

Prof. dr. Guy Parcel (University of Texas Health Science Centre, Houston) 


\section{Table of contents}

$\begin{array}{lll}\text { Chapter } 1 & \text { Introduction } & 7\end{array}$

$\begin{array}{lll}\text { Chapter } 2 \text { First same-sex partner and the Internet } & 15\end{array}$

Chapter 3 Risky debut: high levels of unprotected sex among young gay and bisexual men during their coming-out in the Netherlands

Chapter 4 Longitudinal risk behaviors of a sample of young gay and bisexual men in the Netherlands

Chapter 5 Social-cognitive determinants of condom use in a sample of young gay and bisexual men

Chapter 6 Minority-stress and depressive symptoms in a sample of young gay and bisexual men

Chapter 7 General discussion

References

103

Summary

Nederlandse samenvatting

Acknowledgements

Curriculum Vitae 



\section{Chapter 1 Introduction}

Since the outbreak of the human immunodeficiency virus (HIV), millions of people worldwide have been infected with HIV. In 2007, an estimated 2.5 million people acquired HIV and by the end of 2007 an estimated 33.2 million people were living with HIV [1]. Although the global number of people living with HIV is increasing because of ongoing accumulation of new HIV-infections with longer survival times, the global prevalence of HIV-infection has leveled off in recent years [1]. In most industrialized countries, important risk groups are people who have unprotected sexual intercourse and injecting drug users (IDUs) who share contaminated needles. The predominant risk group among people who have unprotected sexual intercourse is men who have sex with men (MSM). Therefore, interventions are needed to encourage MSM to always practice safe sex. A better understanding of the reasons why MSM engage in sexual risk behavior may guide the development of these interventions. The focus of the present thesis is on young MSM, and more specifically on young MSM who are in the midst of their coming-out. The aim of the present thesis is to describe the results of Outcomes, an online cohort on coming-out and sexual risk behavior of young MSM who were - at study entry - in the midst of their coming-out. The primary focus of the present thesis will be on sexual risk behavior of young MSM. Next to sexual health, a second health problem among MSM is mental health problems as a result of belonging to a sexual minority (see for a review [2]). Therefore, the secondary focus of this thesis will be on mental health outcomes (i.e. depressive symptoms) in young MSM. Before providing an outline of the present thesis, a brief overview of the Outcomes project will be presented. 


\section{HIV-epidemic}

In the Netherlands, the HIV-epidemic is predominantly among men, especially among men who have sex with men (MSM). The proportion of MSM in the annual number of newly diagnosed men with HIV decreased from 58\% in 1996 to 44\% in 2003; thereafter it increased again to $69 \%$ in 2008 [3]. Thus, in the Netherlands, MSM are the most important risk group for HIV-infection. There is a growing body of literature that suggests that younger age is an important risk factor for HIV-infection among MSM [4-6]. Most researchers refer to younger age as MSM younger than 25 years of age. In the United States, the Centre for Disease Control (CDC) monitors rates of HIVinfections among MSM and a recent surveillance report showed that the number of YMSM infected with HIV is increasing, especially among black YMSM [4]. Similar rises in HIV-infections among YMSM has been observed in other Western countries [7-9]. In the Netherlands, no specific data is available on HIV-infections among YMSM. However, recent Dutch MSM Monitoring studies showed that of the MSM who indicated they are HIV-positive, about $5 \%$ is younger than 25 years of age $[5,6]$.

The main route of transmission among MSM is through unprotected anal intercourse (UAI). It is assumed that unprotected oral sex also may also facilitate HIV-transmission but the HIV-transmission risk through unprotected oral intercourse is negligible. In order to control the spread of HIV and other STIs, interventions promoting safer sexual behaviors must be developed. The development of effective interventions requires a thorough understanding of the problem behavior (i.e. unprotected anal intercourse), the target group performing the behavior, and the determinants of the problem behavior. The target group in the present thesis is YMSM who are in the midst of their coming-out process. There are two important yet distinct reasons why we focus on YMSM in the midst of their comingout. First, most coming-out models assume that YMSM will have their sexual debut at some point during their coming-out [10-13]. Second, as already noted above, young age is usually found to be a risk factor for sexual risk-taking behavior. Therefore, it is important to provide 
YMSM as early as possible (i.e. during their sexual debut) with adequate knowledge and skills regarding safe sexual behavior.

\section{Coming-out}

The coming-out process refers to the sexual identity development of lesbians, gays and bisexuals [11, 12, 14-17]. In general, identity development begins with first awareness of same-sex attractions and continues until the individual develops an integrated sense of self as a person with a gay, lesbian or bisexual orientation. Other critical stages include disclosure of sexual identity to others (also referred to as coming-out), first sexual experience with a same-sex partner, and selfacceptance and self-labeling as gay, lesbian or bisexual. Earlier models on the coming-out process hypothesized that progression through these developmental stages occurs in a linear fashion which means that an individual can only progress to the next stage after completion of the previous stage [see for overview 18]. For example, these linear models posit that the first sexual experience with a same-sex partner will occur after the disclosure of sexual identity to others. Recently, linear models have been criticized for failing to recognize the variability in the coming-out process $[16,17]$. For example, research has shown that whereas some gay men report their first same-sex sexual experience prior to disclosing their sexual identity, others report disclosing their sexual identity prior to their first sexual contact with same-sex partners $[10,13,19]$.

Relatively little research has been conducted on the association between sexual risk behavior and the coming-out process with most research focusing on the timing of the coming-out stages (i.e. first same-sex experience before or after first disclosure of sexual identity). For example, some studies showed that MSM who had a sex-centered coming-out (i.e. their first same-sex sexual experience before their first disclosure of their sexual identity) report more lifetime male sex partners and more UAI compared to MSM who had an identitycentered coming-out (i.e. first same-sex experience after their first disclosure) $[10,13]$. Other studies did not observe such an association between coming-out sequence and sexual risk behavior $[20,21]$. There 
is little behavioral data available on the sexual debut of YMSM or on sexual risk behavior of YMSM who are in their coming-out process. It should be noted that the aim of the present thesis is not to test associations between the coming-out process (i.e. the various developmental stages) and sexual risk behavior but to provide insight into the sexual (risk) behaviors of YMSM who are in the midst of their coming-out.

\section{Determinants of sexual risk behavior of MSM}

In order to guide the development of interventions promoting safer sex among MSM, the determinants of sexual risk behavior and condom use have to be known [22]. In general, these determinants can be divided into behavioral and social-cognitive determinants derived from social-cognitive theories. Based on previous research on sexual risk behaviors of MSM in the Netherlands [5, 6, 23], several behavioral factors were identified and incorporated in the present research. Most noteworthy are relationship status, number of lifetime same-sex partners, and HIV-testing. The social-cognitive determinants were derived from social-cognitive theories.

Several social-cognitive theories have been used to explain sexual risk behavior in MSM, most prominently the Theory of Reasoned Action (TRA; [24]) and the Planned Behavior (TPB; [25]), and more recently the reasoned action approach [26]. The TRA assumes that an individual's behavior is influenced by his intention to perform a particular behavior. For example, the TRA assumes that MSM who intend to use condoms when having anal intercourse are more likely to actually use condoms during anal intercourse. The TRA also assumes that behavioral intentions are predicted by the individual's attitudes (which reflect an individual's beliefs regarding the consequences of a specific behavior) and subjective norms (which reflect the perceived approval or disapproval of an individual's social referents regarding the performance of a specific behavior, weighed with the individual's motivation to comply with the referent). For example, the TRA assumes that MSM who express more positive beliefs regarding condom use (e.g. condom use is a good protection against HIV) are 
more likely to have a positive intention to use condoms. In similar vein, MSM who belief that their relevant social referents think that they should always use condoms are more likely to have a positive intention to use condoms. The TPB is an extension of the TRA and incorporates one aspect of Bandura's Social Cognitive Theory (SCT; [27]). The SCT postulates that individuals who belief they possess the skills to perform a certain behavior (termed self-efficacy or perceived behavioral control) are necessary to motivate people into action. For example, MSM who belief they have the skills to negotiate condom use with their sex partner are more likely to have a positive intention to use condoms. The recently proposed reasoned action approach is a further extension of the TRA and the TPB [26]. Research on the predictive value of the TPB has shown that not all persons with a strong intention will carry out the behavior. The reasoned action approach recognizes this intention - behavior gap and explains this gap by stating that the presence of environmental constraints or a lack of actual skills or abilities to perform a behavior (termed "actual control") may prevent people from acting upon their strong intentions. For example, MSM may have a strong intention to always use condoms when having anal sex with a casual partner, but may not act upon this strong intention if no condoms are available (i.e. environmental constraint) or if they do not know how to apply a condom (i.e. lack of skills and abilities). In sum, the reasoned action approach assumes that behavior is best predicted by behavioral intentions and that actual control of the behavior moderates the intention - behavior relationship. Behavioral intentions in turn are best predicted by the determinants attitudes, social norms, and perceived behavioral control. The reasoned action approach also assumes that more distal variables such as demographics, influence intention and behavior through these determinants. The TPB and the reasoned action approach will serve as the theoretical framework for the research described in this thesis (see chapters 5 and 6). 


\section{Mental health in MSM}

There is an abundance of research which shows an association between sexual orientation and mental health outcomes. Lesbians, gays and bisexuals appear to be at greater risk for developing depression or anxiety disorders and to commit suicide [20,28-31]. Meyer has proposed a theoretical framework for understanding the prevalence of mental disorders in lesbian, gays, and bisexuals [2], which will serve as the theoretical basis for chapter six. This framework explains the association between being a sexual minority and increased mental health concerns in terms of minority-stress, defined as additive stress factors due to belonging to a sexual minority. In short, this model posits that both external (i.e. the experience of prejudice events such as discrimination and violence) and internal stress-factors (i.e. concealment of sexual identity, lack of self-acceptance, or internalized homonegativity) contribute to increased levels of stress. At the same time, coping strategies such as being highly involved in the gay scene may ameliorate the effects of minority-stress factors on the mental health of lesbians, gays, and bisexuals [2].

There is evidence that YMSM in the midst of their coming-out are at particular risk for mental health problems [20, 31, 32]. It is assumed that the coming-out process is a stressful time for most YMSM. The mere fact that one realizes that one is attracted to men instead to women may be very stressful for YMSM, especially for those who have a negative or stereotypical perception of gay men (i.e. internalized homonegativity). Moreover, YMSM may decide to conceal their sexual orientation from their parents or friends at school for period of time until they feel ready to disclose their sexual orientation to them. Then, YMSM face the challenge of disclosing their sexual identity to their parents and friends which most YMSM again perceive as a stressful event. In short, YMSM in their coming-out face a number of challenges and these challenges can result in increased feelings of stress. Since the Outcomes study focused on YMSM in their comingout, it provided us with the opportunity to examine the extent to which these minority-stress factors stressors are present in YMSM in their coming-out. Moreover, it provided us with the opportunity to explore 
the potential association between minority-stress factors and mental health problems.

\section{Outcomes}

The Outcomes study is a longitudinal research project which aimed to investigate coming-out and sexual risk behavior of YMSM in the Netherlands. The Outcomes study consists of both qualitative and quantitative research. In the quantitative part of Outcomes, 185 YMSM complete 6 biannual questionnaires on coming-out, sexual (risk) behavior with steady, casual and regular partners, socialcognitive determinants of condom use with casual partners, acculturation (including the Internet) discrimination and mental health. In the qualitative part of Outcomes, 29 YMSM in the midst of their coming-out are interviewed on 6 biannual occasions. The Outcomes study aimed to answer four main research questions:

- What is the rite of passage with respect to sexuality and sexual relations?

- How does risk perception regarding HIV/STIs develop?

- How and to what extent do YMSM after their coming-out attach themselves to gay subcultures?

- What is the role of the Internet in the coming-out process?

The present thesis will only report on the quantitative part of Outcomes. Moreover, in the present thesis not all these research questions will be addressed. The main focus of the present thesis is on sexual behavior and on behavioral and cognitive determinants of sexual risk behavior of YMSM. For a complete overview of the Outcomes project results, the reader is referred to the annual Outcomes reports $[33,34]$.

\section{Thesis outline}

The main objective of the present thesis was to investigate sexual risk behavior of YMSM who are in the midst of their coming-out. A second objective was to explore the association between minority-stress factors 
and mental health outcomes (i.e. depressive symptoms). In chapter 2, condom use at sexual debut will be examined. The rate of UAI with the first same-sex partner will be described and the relationship between meeting place and UAI with the first same-sex partner will be examined. Chapter 3 describes the baseline measurement of sexual (risk) behavior with steady, regular and casual partners. Moreover, correlates of UAI per partner type will be presented in this chapter. In chapter 4, longitudinal data of sexual (risk) behaviors will be described. Also, based on their sexual behavior, YMSM will be classified into three risk groups: consistent no risk, consistent risk and variable risk and behavioral and cognitive correlates of risk group will be presented. In chapter 5, the social-cognitive determinants of condom use with casual partners will be presented. In chapter 6 a study is presented which examined the association between minority-stress factors and mental health outcomes (i.e. depressive symptoms). More specifically, this study aimed to see whether external (i.e. experienced discrimination) and internal (i.e. coming-out related factors) are associated with the report of experiencing symptoms. Finally, in chapter 7 , the results of the foregoing chapters will be discussed and tips for intervention development will be provided. 


\section{Chapter 2 First same-sex partner and the Internet ${ }^{1}$}

There is ample evidence that the Internet is a popular meeting place for gay and bisexual men (e.g. [35-37]). Because of its anonymity the Internet may be especially appealing for young gay and bisexual men (YGBM) in their coming-out (i.e. the process of identity formation and identity integration $[12,15])$. It provides YGBM with a relative safe environment for experimenting with meeting and subsequently having sex with other gay and bisexual men [35, 38]. Two recent studies showed that an increasing number of YGBM nowadays met their first same-sex partner online [35, 39].

The issue whether seeking and meeting same-sex partners online facilitates high risk sexual behaviors is still unresolved. Whereas some studies found evidence that for YGBM seeking same-sex partners online results in increased risk on contracting STI and HIV through a mechanism of more sexual risk behavior (e.g. [36]), other studies found no difference in sexual risk behavior between meeting same-sex partners online versus offline (e.g. [40, 41]).

Considering the Internet's popularity, the question arises whether meeting the first same-sex partner online is associated with more unprotected anal intercourse (UAI). The aim of this study is to examine where YGBM, who are in the midst of their coming-out, meet their first same-sex partner and whether condoms are used during the first episode of anal sex.

\footnotetext{
${ }^{1}$ This chapter is co-authored by Harm Hospers and Gerjo Kok and a modified version
} of this chapter has been submitted for publication under the same title. 


\section{Method}

The Outcomes study is an online cohort study on coming-out and sexual behavior. Between early 2007 and late 2009 respondents complete six biannual questionnaires. The study was approved by the local ethics committee.

The eligibility criteria for the Outcomes study were: being between 16 and 25 years of age ${ }^{2}$, being sexually attracted to men, and first disclosure of sexual attraction in the year prior to the survey. Although coming-out is generally regarded as a process, whereby YGBM progress through several distinct stages (e.g. first realization of same-sex attraction, first same-sex experience, disclosure of sexual attraction to others; $[11,12,15,19]$, in the present study coming-out was defined as the singular event of telling to at least one significant other (e.g. parents, siblings, friends or teacher at school) about attraction to the same sex. Defining coming-out as a singular event enabled us to set clear inclusion criteria. Moreover, it ensured that respondents are still in the midst of their coming-out. It should be noted that by using this working definition, we excluded disclosing sexual identity anonymously (e.g. through online chatting in gay chat sites or through anonymous participation in gay-related Internet forums).

Cohort participants were recruited both offline $(45.9 \%)$ and online $(54.1 \%)$. Offline recruitment took place at various gay venues frequented by YGBM. Details of YGBM (i.e. age, recency of comingout and telephone number) were obtained and eligible participants were contacted by telephone within three working days. Other offline recruitment consisted of placing advertisements promoting the web address of the survey in gay-related media aimed at YGBM. Online recruitment consisted of placing banners with a link to the survey website on various gay-related sites frequented by YGBM and by issuing a press release which was published on various gay news sites. On the website of our survey, interested participants could submit their

\footnotetext{
${ }^{2}$ Although some gay and bisexual youth in the Netherlands nowadays come out before the age of 16 , it was deemed inappropriate to include them in this study since that would have required parental consent.
} 
contact details (e-mail and telephone number) and if they did they were contacted by the primary researcher within three working days. The telephone interview was primarily aimed at verifying inclusion criteria and to clarify the procedure of the study. Eligibility was confirmed by asking interested participants' age, their sexual attraction ( $1=$ only attracted to men, $2=$ mostly attracted to men, $3=$ both attracted to men and women, $4=$ mostly attracted to women and $5=$ only attracted to women) and when and to who they first disclosed their sexual attraction. Next, to ensure that only the intended participants entered the cohort, eligible participants received an e-mail with a personal login name and password which they had to use to login to the website that contained the questionnaire. The telephone interview also provided us with the opportunity to strengthen the commitment of the participants for participation in the cohort study by stressing the importance of the study and by asking their willingness to complete all six questionnaires in the next 2.5 years.

A total of 185 participants (mean age: 18.8 ; $\mathrm{sd}=2.3$; median: 18 , range 16-26), who were in the midst of their coming-out (median number of months after first disclosure of sexual identity: 7) completed their first online questionnaire on coming-out and sexual behavior. The online questionnaire contained the following measures:

Demographics: the demographic characteristics of respondents were assessed using a fill-in-the-blank measure of age, postal code of place of residence (dichotomized into rural versus urban), an educational status checklist (dichotomized into lower versus higher education based on Dutch educational system), a cultural background checklist (dichotomized into Dutch or Western versus Non-Western), living situation checklist (categorized into with parents, with students, alone), and a religious affiliation of respondent and of the respondents' parents checklist (based on measure of importance of religious belief dichotomized into yes versus no).

Sexual history: respondents were asked to indicate the number of life-time same-sex partners, the age at the first same-sex experience and the age of their first same-sex partner. Furthermore, respondents were required to indicate whether their first same-sex experience 
happened before or after their coming-out. With respect to the first episode of anal intercourse, respondents were asked to indicate the meeting place of their first anal same-sex partner (i.e. at school, on the Internet, via friends, in gay bars, in non-gay bars, at YGBM related discussion groups, at sports club, at YGBM organizations, or elsewhere; dichotomized into offline versus online), partner type (steady, regular ${ }^{3}$ or casual partner) and condom use (yes; no). Finally, respondents were required to indicate for a number of STI whether they ever contracted a STI and when they last contracted each STI and whether they were ever tested for HIV and what the result was of their last HIV-test.

Recent sexual behavior: for each type of partner separately (steady, regular and casual partner), respondents were required to indicate the number of partners in the six months preceding the survey, whether they have had sex with each partner, whether they have had receptive and insertive anal intercourse with that partner and whether condoms were used for both receptive and insertive anal intercourse was assessed for steady, regular and casual partners. Sexual behavior with a steady partner was only asked for participants who indicated current involvement in a steady relationship or who broke up with steady partner in the preceding six months. For participants who reported two or more steady partners in the preceding six months, sexual behavior and condom use was asked for the last steady partner. For participants who reported more than one regular partner in the preceding six months, sexual behavior was asked for the regular partner with whom they had had sex most frequently in the preceding six months. A measure of unprotected anal intercourse was constructed per partner type by coding all respondents who reported at least one episode of unprotected receptive or insertive anal sex in the six months preceding the study.

The online questionnaire also included other measures (e.g. coming-out related variables, social-cognitive variables related to

\footnotetext{
${ }^{3}$ A steady partner is defined as someone with whom you have a serious romantic relationship. A regular partner is defined as someone with whom you have regularly sex, but without being involved in a serious romantic relationship.
} 
condom use, acculturation and discrimination). Participants receive $€ 10$ for completing each questionnaire, and were informed that they would receive an additional bonus of $€ 40$ if they complete all six questionnaires.

For the current study, the following measures are used: meeting place, partner type, and condom use with the first anal same-sex partner. The analyses are restricted to respondents for whom complete data are available on the first episode of anal intercourse ( $N=95)$. Chisquared tests were used to detect significant differences in UAI with first anal same-sex partner and meeting place (offline versus online) and partner type (steady, regular, casual).

\section{Results}

Respondents were very active online; only two respondents never visited gay sites on the Internet. Of the respondents who did visit gay sites, $52 \%$ visited gay sites daily, $22 \%$ between 2 and 5 times a week, and $26 \%$ once a week or less. Of the 141 respondents who completed all four questionnaires, $82 \%(\mathrm{~N}=116)$ have had experience with anal intercourse. Due to technical reasons, only of 95 respondents complete data are available. No significant difference were observed between respondents for whom complete and non-complete data are available (all p's >.05). Therefore, it is assumed that attrition occurred at random. Of the 95 respondents for whom complete data were available, $45 \%$ reported UAI with their first same-sex partner. Almost half of these respondents met their first same-sex partner online $(47 \%)$, $15 \%$ through friends, $12 \%$ at school, $12 \%$ in a gay bar, $2 \%$ through sports, $1 \%$ in a non-gay bar, and $12 \%$ elsewhere (e.g. theatre, neighborhood, fashion show, etc.). There was no significant difference in UAI with first same-sex partner when met offline $(52 \%)$ versus online $\left(38 \% ; \chi^{2}=1.93 ; \mathrm{p}>.05\right.$; see Table 2.1$)$. 
Table 2.1: Meeting place and partner status of first same-sex partner and rate of UAI (N=95).

\begin{tabular}{lll}
\hline & Percentage (N) & $\%$ UAI \\
\hline Meeting place & & \\
Online & $47 \%(\mathrm{~N}=45)$ & $38 \%$ \\
Offline & $53 \%(\mathrm{~N}=50)$ & $52 \%^{*}$ \\
Partner status & & \\
Steady & $45 \%(\mathrm{~N}=43)$ & $37 \%$ \\
Regular & $28 \%(\mathrm{~N}=27)$ & $56 \%$ \\
Casual & $26 \%(\mathrm{~N}=25)$ & $48 \% * *$ \\
\hline$\chi^{2}=1.93, \mathrm{p}>.05 ;{ }^{* * *} \chi^{2}=2.6, \mathrm{p}>.05$ & &
\end{tabular}

Of the $45 \%$ of respondents who indicated that their first episode of anal intercourse was with a steady partner, $37 \%$ reported UAI. Of the $28 \%$ of respondents who indicated the first episode was with a regular partner, $56 \%$ reported UAI. Finally, $26 \%$ of the respondents had the first episode of anal intercourse with a casual partner, of whom $48 \%$ reported UAI. No significant differences in rates of UAI per partner type were observed $\left(\chi^{2}=2.36 ; \mathrm{p}>.05\right.$; Table 2.1$)$.

\section{Discussion}

First, it should be noted that the Outcomes study has a limited sample size. Moreover, the study sample consists of YGBM who are in the midst of their coming-out. Finally, in contrast to other samples (e.g. $[36,39])$, our sample was both recruited offline and online. Therefore, our results should be interpreted with caution.

The main aim of this study was to examine where YGBM meet their first same-sex partner and whether meeting place was associated with sexual risk behavior with that first same-sex partner. The results confirm previous observations that many YGBM are active on the Internet and that many - but not all - meet their first same-sex partner online [35-37, 39]. Moreover, almost half of the respondents did not use a condom during their first episode of anal intercourse. Third, although partner status (i.e. steady partner) is considered to be an 
important risk factor for HIV-infection [42], partner status of the first same-sex partner was not associated with UAI during the first episode of anal intercourse. However, it is possible that levels of UAI increase with relationship duration [43]. Finally, in contrast to other studies (e.g. [36, 44]), no significant difference in sexual risk behavior between online and offline first same-sex partners was observed. However, much of the literature on the role of the Internet on sexual risk behavior focuses on "meeting online for sex". This raises the question whether YGBM were intentionally meeting other YGBM online for sex when they met the first same-sex partner (i.e. high risk) or just for socializing (i.e. low risk). Future studies should determine whether the context of meeting first same-sex partners online influences the decision to engage in UAI with the first same-sex partner.

In conclusion, the finding that a considerable proportion of YGBM engaged in UAI with first same-sex partner calls for specific HIV-prevention activities encouraging YGBM to use condoms during the first episode of anal intercourse. Furthermore, although meeting the first same-sex partner online was not associated with more risk behavior, the finding that many YGBM are active online - even in the midst of their coming-out - suggests that these HIV-prevention activities should also be delivered online. 



\section{Chapter 3 Risky debut: high levels of unprotected sex among young gay and bisexual men during their coming-out in the Netherlands ${ }^{4}$}

Men who have sex with men (MSM) continue to be at risk of acquiring HIV through unprotected anal intercourse (UAI). In the last decade about $50 \%$ to $60 \%$ of new HIV-infections were diagnosed among MSM each year, both in the Netherlands [3], and other Western countries $[1,7,8,45]$. Thus, MSM - at least in Western countries - are still the largest risk group for acquiring HIV.

Research consistently shows that younger age is an important risk factor for sexual risk-taking, both in the Netherlands [5, 6, 23] and elsewhere [46-53], and it has been suggested that YGBM are in part responsible for recent increases in HIV-infections and other STI [48, 49, 52, 54, 55]; but see also [56]). However, in most samples that report on sexual (risk) behavior, YGBM in their coming-out process are underrepresented. For example in 2003, the Dutch MSM Monitoring Study showed that of the 1160 men who indicated their age and their age of coming-out, 216 participants were aged 25 or younger, and of those only 36 had their coming-out in the year preceding the survey [57].

A limited number of studies specifically addressed sexual behavior of YGBM in their coming-out. One study found that YGBM (aged 15-29 years) who had disclosed their sexual identity to others report more sexual partners and higher rates of UAI compared to nondisclosers [58]. Two retrospective studies showed that MSM who had their first same-sex experience before their coming-out (defined as

\footnotetext{
${ }^{4}$ This chapter is co-authored by Harm Hospers and Gerjo Kok and a modified version of this chapter has been submitted for publication under the same title.
} 
having their first same-sex experience before they first disclosed their sexual orientation to one significant other) report more lifetime sex partners and more UAI compared to MSM who had their first samesex experience after their coming-out $[10,13]$. On the other hand, one prospective study showed no association between sequence of disclosure and UAI (i.e. [20]). Finally, one recent retrospective study by Friedman and colleagues [21] showed that timing of coming-out (i.e. early, middle or a late start of disclosing sexual identity to significant others) was unrelated to any unprotected sex in adulthood. However, to date little behavioral data is available on the start of YGBM's sexual trajectory. Moreover, little is known about the sexual risk behavior of YGBM who are in the midst of their coming-out (i.e. who start to disclose their sexual identity to others). Therefore, the first aim of the present study is to provide behavioral data on sexual risk behaviors with the first same-sex partner and with recent same-sex partners of YGBM in their coming-out.

Several studies have found differential risk taking behaviors based on partner type. MSM are more likely to engage in sexual risk behavior with steady partners compared to casual partners [46, 59-61]. For example, in Dutch MSM Monitoring studies, the rate of UAI with steady partners for YGBM is approximately $60 \%$, and approximately $35 \%$ for casual partners [5, 6, 23]. However, surveys and research on sexual risk behavior of MSM usually differentiate between partner type (i.e. sex with steady and with casual partners). Steady partners are defined as main partners or romantic lovers, while casual partners are defined as one night stands or new casual partners [49, 52, 58, 62, 63]; see [64] for other partner categories). Two recent Dutch MSM Monitoring Studies [5, 23] suggest yet another relevant partner category. These studies found that almost half of respondents indicated they had had sex before with their last casual partner; in the majority of cases more than three times before. Moreover, having had sex before with the last casual partner was significantly related to more unprotected sex, especially among YGBM. Of the YGBM who never had sex before with their last casual partner, $13 \%$ had unprotected sex, 
while of the YGBM who have had sex three times or more before, $40 \%$ had unprotected sex.

In order to facilitate HIV prevention activities for YGBM, the present study aims to provide behavioral data on the sexual risk behavior of YGBM who are in the midst of their coming-out. As a result of the findings on partner type discussed above, we decided to explicitly differentiate between steady partners (i.e. someone with whom respondents have a serious romantic relationship), regular partners (i.e. someone with whom respondents regularly have sex, but without being involved in a serious romantic relationship), and casual partners (i.e. someone with whom respondents never had sex before). First, baseline data on sexual risk behavior with first same-sex partner and with recent (i.e. in the six months preceding the survey) steady, casual and regular partners from an online longitudinal cohort study of Dutch YGBM in their coming-out will be presented. Second, associations between demographic and behavioral characteristics (i.e. sexual history variables) and rates of UAI by partner type will be explored.

\section{Methods}

The Outcomes study is an online cohort study on coming-out and sexual behavior. Between early 2007 and late 2009 participants complete six biannual questionnaires. The data included in the present analysis are from the first (i.e. baseline) measurement. The study was approved by the ethics committee of the Faculty of Psychology and Neuroscience, Maastricht University.

The eligibility criteria for the Outcomes study were: being between 16 and 25 years of age ${ }^{5}$, being sexually attracted to men, and first disclosure of sexual attraction in the year prior to the survey. Although coming-out is generally regarded as a process, whereby YGBM progress through several distinct stages (e.g. first realization of same-sex attraction, first same-sex experience, disclosure of sexual

\footnotetext{
${ }^{5}$ Although some gay and bisexual youth in the Netherlands nowadays come out before the age of 16 , it was deemed inappropriate to include them in this study since that would have required parental consent.
} 
attraction to others; $[11,12,15,19]$, in the present study coming-out was defined as the singular event of telling to at least one significant other (e.g. parents, siblings, friends or teacher at school) about attraction to the same sex. Defining coming-out as a singular event enabled us to set clear inclusion criteria. Moreover, it ensured that respondents are still in the midst of their coming-out. It should be noted that by using this working definition, we excluded disclosing sexual identity anonymously (e.g. through online chatting in gay chat sites or through anonymous participation in gay-related Internet forums).

Cohort participants were recruited both offline $(45.9 \%)$ and online $(54.1 \%)$. Offline recruitment took place at various gay venues frequented by YGBM. Details of YGBM (i.e. age, recency of comingout and telephone number) were obtained and eligible participants were contacted by telephone within three working days. Other offline recruitment consisted of placing advertisements promoting the web address of the survey in gay-related media aimed at YGBM. Online recruitment consisted of placing banners with a link to the survey website on various gay-related sites frequented by YGBM and by issuing a press release which was published on various gay news sites. On the website of our survey, interested participants could submit their contact details (e-mail and telephone number) and if they did they were contacted by the primary researcher within three working days. The telephone interview was primarily aimed at verifying inclusion criteria and to clarify the procedure of the study. Eligibility was confirmed by asking interested participants' age, their sexual attraction ( $1=$ only attracted to men, $2=$ mostly attracted to men, $3=$ both attracted to men and women, $4=$ mostly attracted to women and $5=$ only attracted to women) and when and to who they first disclosed their sexual attraction. Next, to ensure that only the intended participants entered the cohort, eligible participants received an e-mail with a personal login name and password which they had to use to login to the website that contained the questionnaire. The telephone interview also provided us with the opportunity to strengthen the commitment of the participants for participation in the cohort study by stressing the importance of the 
study and by asking their willingness to complete all six questionnaires in the next 2.5 years.

A total of 185 participants, who were in the midst of their coming-out (median number of months after first disclosure of sexual identity: 7) completed their first online questionnaire on coming-out and sexual behavior. The online questionnaire contained the following measures:

Demographics: the demographic characteristics of respondents were assessed using a fill-in-the-blank measure of age, postal code of place of residence (dichotomized into rural versus urban), an educational status checklist (dichotomized into lower versus higher education based on Dutch educational system), a cultural background checklist (dichotomized into Dutch or Western versus Non-Western), living situation checklist (categorized into with parents, with students, alone), and a religious affiliation of respondent and of the respondents' parents checklist (based on measure of importance of religious belief dichotomized into yes versus no).

Sexual history: respondents were asked to indicate the number of life-time same-sex partners, the age at the first same-sex experience and the age of their first same-sex partner, whether the first same-sex experience involved anal intercourse (yes/no) and whether a condom was used during their first same-sex anal episode (yes/no). Furthermore, respondents were required to indicate whether their first same-sex experience happened before or after their coming-out (from now on referred to as coming-out sequence). Finally, respondents were required to indicate for a number of STI whether they ever contracted a STI and when they last contracted each STI and whether they were ever tested for HIV and what the result was of their last HIV-test.

Recent sexual behavior: for each type of partner separately (steady, regular and casual partner), respondents were required to indicate the number of partners in the six months preceding the survey, whether they have had sex with each partner, whether they have had receptive and insertive anal intercourse with that partner and whether condoms were used for both receptive and insertive anal intercourse was assessed for steady, regular and casual partners. Sexual behavior 
with a steady partner was only asked for participants who indicated current involvement in a steady relationship or who broke up with steady partner in the preceding six months. For participants who reported two or more steady partners in the preceding six months, sexual behavior and condom use was asked for the last steady partner. For participants who reported more than one regular partner in the preceding six months, sexual behavior was asked for the regular partner with whom they had had sex most frequently in the preceding six months. A measure of unprotected anal intercourse was constructed per partner type by coding all respondents who reported at least one episode of unprotected receptive or insertive anal sex in the six months preceding the study.

The online questionnaire also included other measures (e.g. coming-out related variables, social-cognitive variables related to condom use, acculturation and discrimination) which will be presented elsewhere. Participants receive $€ 10$ for completing each questionnaire, and were informed that they would receive an additional bonus of $€ 40$ if they complete all six questionnaires.

Data analysis: the two main goals of the current research are to provide behavioral data on the sexual behaviors of YGBM who are in the midst of their coming-out and to investigate the relationship between demographic and sexual history variables on the one hand, and UAI per partner type on the other hand (i.e. UAI with first samesex partner, and UAI with recent steady, regular and casual partners). Therefore, first basic frequencies of UAI are calculated per partner type. Next, point biserial correlations are calculated between UAI with first same-sex partner and UAI per partner type on the one hand, and demographics (age, educational level, and urbanization) and sexual history variables (i.e. age at first same-sex experience, coming-out sequence (i.e. first same-sex experience before or after coming-out), and number of lifetime sex partners) on the other hand. For first samesex partner, the correlation between age of the first same-sex partner and UAI with that first same-sex partner is calculated as well. Moreover, for steady partner, the correlation between relationship duration based on a median split and UAI with steady partner is 
calculated as well. Finally, four separate multivariate logistic regression analyses were performed to further investigate relationships between demographic and sexual history variables and UAI per partner type.

\section{Results}

Respondents' mean age was 18.8 (sd 2.3; median 18; range 16-26). Seventy-two percent of respondents lived at home with their parents, $21 \%$ lived with students, and $7 \%$ lived alone, and almost half of the respondents lived in urban areas $(48 \%)$. Sixty-two percent attended or completed higher education. The vast majority had a Dutch or Western cultural background $(93 \%)$. Finally, $16 \%$ of respondents indicated a religious affiliation and $27 \%$ of the respondents' parents had a religious affiliation.

Sexual history: $12 \%(\mathrm{~N}=22)$ of participants had not (yet) engaged in same-sex behavior. Of the 163 participants who did have same-sex experience, $98 \%$ ever experienced mutual masturbation, $98 \%$ ever had oral sex and $69 \%$ ever had anal intercourse. The mean age at which respondents had their first same-sex experience was 16.6 years (sd 2.6; median 17), while the mean age of their first same-sex partner was 18.7 (sd 4.9; median 18). Eight participants (4.3\%) ever contracted an STI and 31 (16.8\%) were ever tested for HIV, with 30 participants being HIV-negative and 1 participant who never learned his test result. Of the participants who had same-sex experience, $66 \%$ $(\mathrm{N}=108)$ indicated that they had their first same-sex experience before their coming-out. The average number of lifetime same-sex partners was 5.2 (sd 4.9; median 4), with $20 \%$ reporting one same-sex partner, $17 \%$ reporting two partners, and $63 \%$ reporting three or more samesex partners. Of the participants who ever had anal intercourse, $43 \%$ $(\mathrm{N}=48)$ reported unprotected anal intercourse (UAI) with their first same-sex partner. 
Table 3.1 Sexual behavior in preceding 6 months.

\begin{tabular}{lccc}
\hline & $\begin{array}{c}\text { Steady partners } \\
(\mathrm{N}=87)\end{array}$ & $\begin{array}{c}\text { Regular partners } \\
(\mathrm{n}=84)\end{array}$ & $\begin{array}{c}\text { Casual Partners } \\
(\mathrm{n}=76)\end{array}$ \\
\hline No sex & $8 \%$ & - & - \\
No anal intercourse & $33 \%$ & $40 \%$ & $43 \%$ \\
Protected anal intercourse & $16 \%$ & $27 \%$ & $24 \%$ \\
Unprotected anal intercourse & $43 \%$ & $33 \%$ & $33 \%$ \\
\hline
\end{tabular}

Recent sexual behavior (see Table 3.1): 47\% (N=87) of the participants reported a steady partner in the preceding six months. Of these, $59 \%$ $(\mathrm{N}=51)$ had anal intercourse, of whom $73 \%(\mathrm{~N}=37)$ reported UAI (i.e. $43 \%$ of YGBM with steady partners). The mean relationship duration was 6.1 months (sd 7.9; median 3).

Almost half of participants $(45 \%, \mathrm{~N}=83$ ) reported sex with regular partners in the preceding six months. Of these, $61 \%$ had anal intercourse, of whom $55 \%$ reported UAI (i.e. $33 \%$ of YGBM with regular partners). Finally, $41 \%$ ( $\mathrm{N}=76)$ of participants reported sex with casual partners in the preceding six months. Of these, $57 \%$ had anal intercourse with a casual partner, of whom $58 \%$ reported UAI (i.e. $33 \%$ of YGBM with casual partners).

Correlates of UAI: correlations between UAI with first same-sex partner and UAI with recent steady, regular and casual partner and demographics ${ }^{6}$ and sexual history variables are presented in Table 3.2. Only respondent's age at first same-sex experience was significantly correlated with UAI with first same-sex partner $(r=.30 ; \mathrm{p}<.05)$. For recent steady partner, there was a significant correlation between relationship duration (based on median split) and UAI ( $\mathrm{r}=.22 ; \mathrm{p}<.05)$, indicating that when a relationship lasts for four months or more, respondents are more likely to report UAI with their steady partner. No other significant correlations were observed for recent steady partner. No significant correlations were observed for UAI with recent

\footnotetext{
${ }^{6}$ Living situation, cultural background and religious affiliation of participant and parents could not be included in the logistic regression analyses because of insufficient variance.
} 
regular partner. Finally, there was a significant correlation between the number of lifetime same-sex partners and UAI with recent casual partner $(\mathrm{r}=.37 ; \mathrm{p}<.01)$, indicating that reporting higher numbers of lifetime same-sex partners is associated with increased likelihood of UAI with recent casual partner.

A multivariate logistic regression analysis with UAI with first same-sex partner as dependent variable and demographics (i.e. age, level of education, and urbanization), and sexual history variables (i.e. age at first same-sex experience, age of first same-sex partner, comingout sequence and number of lifetime same-sex partners) as predictors showed that participants who experienced their first same-sex episode at a younger age were more likely to report UAI with their first samesex partner $(\mathrm{aOR}=1.41,95 \%$ CI $1.11-1.78 ; \mathrm{p}<.01)$. No significant associations with demographics or other sexual history variables were observed for UAI in the preceding six months with steady, regular or casual partners. However, the association between relationship duration and UAI with recent steady partner reached borderline significance, indicating that respondents involved in a steady relationship for four months or longer report more UAI (54\%) with their steady partner compared to participants with a relationship shorter than four months (32\%; aOR 2.7; 95\% CI .97- 7.2; p <.06). There was also a borderline significant positive relationship between number of lifetime same-sex partners and UAI with recent casual partners, indicating that reporting higher number of lifetime same-sex partners is associated with increased odds on UAI with recent casual partner (aOR 1.12; $95 \%$ CI .99-1.2; p <.06). 


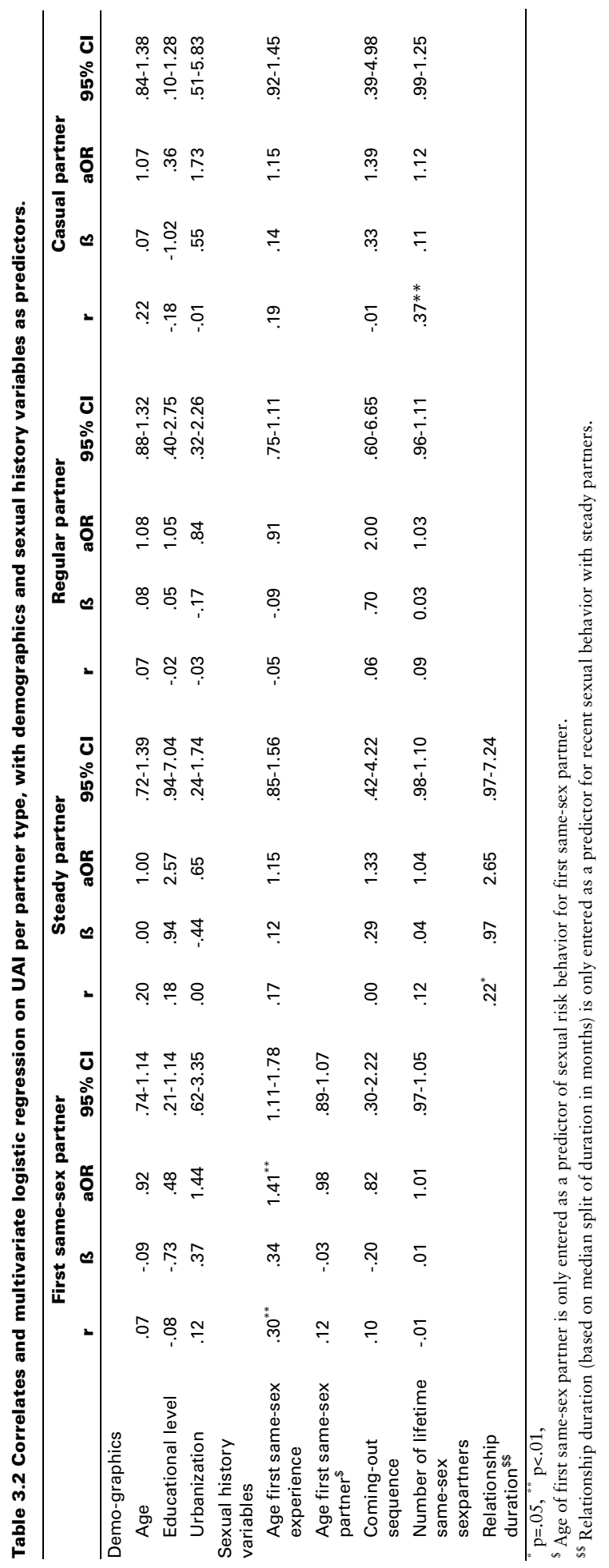




\section{Discussion}

The Outcomes study shows that when YGBM have their first episode of anal sex, a considerable number do not use condoms. Furthermore, becoming sexually active at a younger age increases the odds of UAI with the first same-sex partner. Later on - in the midst of their comingout - substantial numbers have unprotected sex with steady, regular and casual partners. We observed no significant association between demographic or sexual history variables on the one hand and unprotected sexual behavior in the preceding six months for all three partner types on the other hand. Finally, our results show that almost half of YGBM report regular partners (i.e. partners with whom YGBM have regularly sex without being involved in a serious romantic relationship), indicating that regulars thus seem to be a relevant category of sexual partners.

The Outcomes study shows that of the respondents who have had anal sex, about half report condom use the first time they had anal sex. This percentage is lower compared to Dutch heterosexual youth, of whom more than $75 \%$ report condom use during their sexual debut [65]. This finding is a challenge for prevention. Prevention messages urge YGBM to protect themselves against HIV. Our results could indicate that these messages may not convince YGBM to use condoms. Alternatively, it may also be that YGBM have not been exposed to these messages. More research into the circumstances and dynamics of the first instance of anal intercourse may guide strategies to promote condom use for this target group. The finding that the majority of our sample had their first same-sex experience before their coming-out (i.e. before they disclosed their sexual identity to significant others) may ask for strategies that reach young men before they have their coming-out, for example through websites that provide information on coming-out issues.

The Outcomes study also showed that YGBM in their comingout report differential risk taking behaviors per partner type. The levels of recent UAI with casual partners are strikingly similar with UAI levels of their older counterparts $[5,6,9,66]$. For example, in three recent 
Dutch MSM Monitoring studies, the rate of UAI with casual partners for older MSM is approximately $35 \%$ [5, 6, 23]. Furthermore, the association between reporting a higher number of same-sex partners and UAI with casual partners is also a typical finding in HIV-risk research, both in young and older MSM [5, 6, 20, 23, 38, 46, 67-70]. There is also a remarkable difference between our sample and older MSM: in our sample fewer respondents had anal sex with their casual partner but those who did have anal sex report more UAI. Other studies have also shown that younger age (which does not necessarily imply young age), is related to risk behavior [5, 23, 46, 47, 49-53]. However, this is one of the first studies to report high levels of unprotected sex with casual partners of young gay men in the midst of their coming-out. These results warrant that interventions should be developed, based on behavioral determinants that are related to this risk behavior, to inform and motivate this target group to have safer sex.

The levels of recent UAI with steady partners are lower compared to other studies $[5,9,66]$. However, these levels tend to increase with relationship duration, a finding which corroborates previous research [43]. For example, research reporting from a sample of younger MSM (median age 23 years) showed that of the men reporting anal sex with their steady partner, $41 \%$ had UAI when the relationship duration was less than 9 months, compared to $88 \%$ when the relationship lasted for 9 months or longer [43]. Our findings may indicate that YGBM similar to older MSM [71] - use 'negotiated safety' to reduce HIV transmission risks [42, 72]. Negotiated safety is a strategy where sexual partners in steady relationships practice UAI provided that both partners are HIV-negative, and that both partners make clear agreements about safe sex outside their relationship. However, practicing negotiated safety requires both partners to take an HIV-test and the observed low HIV-testing rates indicate that YGBM in our sample are either not practicing negotiated safety yet or are practicing negotiated safety without an HIV-test. Therefore, and because relationship turnover is higher among younger compared to older 
MSM [43], it may be best to advise young MSM to always practice safe sex with their steady partner.

Regular partners are common among YGBM in our sample; in fact more participants had sex with regular partners than with casual partners. The level of UAI with regular partners was similar to that with casual partners. In contrast, the Dutch MSM Monitoring studies consistently show significantly higher levels of UAI among regular partners compared to new casual partners [5,23]. This may imply that levels of UAI with regular partners may increase as younger MSM get older. Alternatively, the similarity in rates of UAI may also suggest that YGBM are not able to differentiate between casual and regular partners or it may suggest that casual partners become regular partners. However, very little is known about the dynamics of sex with regular partners, and more research is needed to understand differences between casual and regular partners. Also, more research is needed to understand reasons for unprotected sex with regular partners in order to better address this issue in prevention.

We found no association between demographic variables and sexual risk behavior with steady, casual or regular partners in the preceding six months. In other studies, race/ethnicity is usually related to sexual risk behaviors $[53,58]$. However, these studies are mainly conducted in the USA, which has a relatively diverse ethnic population [73]. In contrast, the Dutch population is relatively homogenous (i.e. in $20077 \%$ of the Dutch population had a non-western cultural background [74], similar to our sample of YGBM). Furthermore, sexual risk behaviors of YGBM were unrelated to sexual history variables such as age of first same-sex partner or having had the first episode of same-sex before or after coming-out. More likely, predictors of UAI can be found on a motivational/cognitive level such as intention to use condoms [25] or the willingness to engage in UAI (i.e. behavioral willingness; $[75,76]$. In a sense that is good news, since motivational factors are more amenable to change than - for example - educational level or religious beliefs.

The Outcomes study has several limitations. First, because the Netherlands is known to be a fairly liberal country with respect to 
homosexuality [77], some YGBM may have their coming-out at an age younger than 16. Second, recruitment took place at Internet sites and venues that cater to YGBM and this may have resulted in an overrepresentation of YGBM who are already comfortable with their sexual identity. A third limitation is that not all factors which have been found to be associated with sexual risk behaviors were assessed in the current study (e.g. illicit drug use prior or during sex [20], personality factors such as impulsivity and sexual sensation seeking $[20,46]$, family and partner violence $[78,79]$, childhood sexual abuse [20]). Finally, the data presented here are cross-sectional and therefore the results presented here should be interpreted with caution.

In conclusion, this study contributes to the understanding of sexual history and recent sexual behavior of YGBM in the midst of their coming-out. The present study showed that a considerable proportion of YGBM start their sexual career unsafe and continue to have unprotected sex throughout their coming-out. Moreover, we have shown that including regular partners when assessing sexual risk behavior advances our knowledge about sexual risk behaviors of YGBM. Prevention activities in the Netherlands usually target all MSM $[80,81]$. However, based on the Outcomes study, we recommend the development of preventive efforts specifically tailored for YGBM in their coming-out. More specifically, preventive measures are needed to inform YGBM about safe sex before they start practicing same-sex and about subsequent sexual practices and risk with steady, casual and regular partners. 


\section{Chapter 4 Longitudinal risk behaviors of a sample of young gay and bisexual men in the Netherlands ${ }^{7}$}

Annual cross-sectional behavioral surveys conducted in the

Netherlands show that in the last decade about $30 \%$ of men who have sex with men (MSM) report any unprotected anal intercourse (UAI) with casual partners in the six months preceding assessment $[5,6,23]$. Similar rates of UAI with casual partners have been observed in surveys and studies conducted elsewhere (e.g. [82-84]). Recently, baseline data on sexual risk behaviors from a cohort of young gay and bisexual men (YGBM), the same cohort as described in the current paper, showed that of the respondents who had casual or regular partners in the six months preceding the first data wave, 33\% had UAI [85]. A first objective of the present paper is to report rates of UAI at four biannual data waves and to examine longitudinal patterns of sexual risk behaviors of YGBM who were - at study entry - in the midst of their coming-out. These rates of UAI per data wave and the longitudinal patterns of sexual risk behavior will provide information on how many YGBM use condoms consistently (consistent no risk group), never (consistent risk group) or occasionally (variable risk group).

A second objective of this descriptive study is to investigate potential correlates of longitudinal sexual risk behavior in order to facilitate intervention development [86]. More specifically, two classes of correlates will be examined: behavioral and cognitive variables. With respect to behavioral correlates, several studies have found associations between number of lifetime same-sex partners and UAI in

\footnotetext{
7 This chapter is co-authored by Harm Hospers and Gerjo Kok and a modified version of this chapter has been submitted for publication under the same title.
} 
that reporting more lifetime same-sex partners is associated with increased odds on UAI and HIV-infection [20, 38, 46, 67, 68, 70, 87, 88]. Furthermore, recent or repeated HIV-testing has also been associated with more sexual risk behavior among YGBM [62]. With respect to cognitive correlates, several studies have shown that HIVoptimism is related to UAI and HIV-infection [89-91]. The rationale is that recent advances in the treatment of HIV (i.e. the availability of highly active antiretroviral therapy (HAART)) may have led to a diminished concern about the necessity to always practice safe sex. Although several cross-sectional studies have found significant associations between HIV-optimism and sexual risk behavior [92, 93], longitudinal studies seem to indicate that HIV-optimism is more likely the result of sexual risk behavior instead of preceding sexual risk behavior [61, 82, 91, 94]. A second potential cognitive correlate of sexual risk behavior is intention to use condoms, which is assumed to be the most proximal determinant of behavior [25]. Recent research has shown that the intention to always use condoms predicts prospective condom use reasonably well $[20,95,96]$.

In sum, the aim of the present study is twofold. First rates of UAI per data wave and longitudinal patterns of sexual risk behavior will be examined and second potential behavioral and cognitive correlates of longitudinal risk behavior will be explored.

\section{Methods}

The Outcomes study is an online cohort study on coming-out and sexual behavior. Between early 2007 and late 2009 participants complete six biannual questionnaires. The data included in the present analysis are from the first four data waves. The study was approved by the ethics committee of the Faculty of Psychology and Neuroscience, Maastricht University. The methods of the Outcomes study have been described in detail in chapter 2 and 3.

Cohort participants were recruited both offline $(45.9 \%)$ and online $(54.1 \%)$. Offline recruitment took place at various gay venues frequented by young MSM and by placing advertisements promoting the survey website in gay-related media aimed at young MSM. Online 
recruitment consisted of placing banners with a link to the survey website on various gay news sites frequented by young MSM. A total of 185 participants completed their first online questionnaire on coming-out and sexual behavior. Of these 185 respondents, $88 \%$ $(\mathrm{N}=162)$ completed their second questionnaire, $85 \%(\mathrm{~N}=158)$ their third questionnaire and $82 \%(\mathrm{~N}=152)$ their fourth questionnaire. In total, $76 \%(\mathrm{~N}=141)$ of respondents completed all four questionnaires. Each online questionnaire covered an extensive range of topics including: demographics, sexual history measures, and recent sexual behavior with steady, regular and casual partners. The questionnaire also included other measures (e.g. coming-out, social-cognitive determinants of condom use, acculturation and discrimination) which will be presented elsewhere. Participants receive $€ 10$ for completing each questionnaire, and will receive an additional bonus of $€ 40$ if they complete all six questionnaires.

\section{Dependent variables}

The dependent variables were coded similar to a previous study on longitudinal patterns of risk behaviors [97]. To explore cross-sectional sexual behavior patterns in four waves of Outcomes, sexual behavior with steady, regular and casual partners was combined to form one behavioral index: $1=$ no sex partners or no anal intercourse, $2=$ protected anal intercourse, $3=$ unprotected anal intercourse. To assess longitudinal patterns of sexual risk behaviors, this behavioral index was further dichotomized into safe (i.e. no partners or no sex or no anal intercourse or protected anal intercourse) versus unsafe (i.e. unprotected anal intercourse). Based on this dichotomous index, there are 16 possible combinations over four waves $\left(2^{4}\right)$, which were classified into three risk groups:

- Consistent no risk (no partner, no sex, no anal intercourse or no UAI at all four data waves)

- Consistent risk (UAI at all four data waves)

- Variable risk (any combination of no risk and UAI over four data waves) 


\section{Independent variables}

The current analyses used nine independent variables. Two demographic variables ${ }^{8}$ measured at baseline: age and educational level (dichotomized into higher versus lower). Two sexual history variables measured at baseline: number of lifetime same-sex partners (dichotomized based on a median split into $\leq 3$ versus $\geq 4$ ) and ever tested for HIV (no/yes). Finally, five cognitive variables related to HIVoptimism and behavioral intention to use condoms measured at baseline were used (for detailed description, see below).

HIV-optimism was measured by 12 items about HIV-treatment with four items measuring the sub-scale "HIV-treatment optimism", four questions measuring the sub-scale "Need for safe sex in light of HAART" (labeled "safe sex necessity"), and four questions measuring the sub-scale "Difficulty practicing safe sex in light of HAART" (labeled "safe sex fatigue"). Responses were on a five point scale ranging from completely disagree to completely agree. An example of a question about HIV-treatment optimism is: "With the availability of HIV-treatment, I am less concerned about becoming HIV-positive". A reliability analysis showed that one item (i.e. "When HIV-positive and using HIV-treatment, you are less infectious”.) did not scale with the sub-scale and was therefore deleted. Cronbach's alpha of the final subscale is .62. An example question of the "Safe sex necessity" scale is: "With the availability of HIV-treatment, there is less need to use condoms during anal sex". A reliability analysis showed that one item (i.e. "As long as HIV is incurable, using condoms during episodes of anal sex is the best means to have sex") did not scale with the sub-scale and was therefore deleted. Cronbach's alpha of the final sub-scale is .64. An example question of the "Safe sex fatigue" scale is: "I am tired of practicing safe sex all the time". Cronbach's alpha of the final subscale is .83. Cronbach's alpha of the whole scale (i.e. containing 10 items) is .80 .

\footnotetext{
${ }^{8}$ Other demographics such as living situation and religious beliefs could not be used in the current analyses because of insufficient variance. However, visual inspection of contingency tables did not indicate existence of associations between these demographics and the dependent variable.
} 
Behavioral intention was measured by two questions, I intend to and I expect to. For example: "Imagine you have anal sex with a new partner in the next six months. Do you intend to use a condom?". Responses were on a seven point scale ranging from most certainly not to most certainly. The two items were highly correlated $(\mathrm{r}=.84, \mathrm{p}<.01)$ and therefore summed and averaged to form one general intention measure. Because this general intention is very positive and because $51 \%$ of the respondents had a maximal intention, intention was dichotomized by categorizing 'most certainly' as high intention and all other responses as lower intention.

\section{Data analyses}

First, to check whether respondents who dropped-out (i.e. drop-outs consists of respondents who only completed the first questionnaire and of respondents who completed the first and one or more but not all of the follow-up questionnaires) of the Outcomes study differ on demographics and behavioral correlates, data from respondents who completed all four questionnaires will be compared with respondents who dropped-out from the study using T-tests and Chi-square tests. Next, descriptive statistics about demographics, behavioral correlates and consistent condom use will be obtained. Changes in rates of sexual behaviors at each of four data waves will be tested using a Chi-square analysis. Finally, in order to explore potential correlates of consistent condom use (i.e. risk group) univariate multinomial logistic regression analyses will be performed with risk group as dependent variable and age, educational background, number of lifetime same-sex partners $(\leq 3$ versus $\geq 4$ ), HIV-testing, HIV-treatment optimism and behavioral intention (high versus lower) measured at baseline as covariates.

\section{Results}

Respondents' mean age at baseline was 18.8 (sd 2.3; median 18; range 16-26). Seventy-two percent of respondents lived at home with their parents, $21 \%$ lived with students, and $7 \%$ lived alone, and almost half of the respondents lived in urban areas $(48 \%)$. Sixty-two percent attended or completed higher education. Mean score on the total HIV- 
optimism scale at baseline was 1.88 ( $\mathrm{sd}=.63$; range 1-3.8), and of the HIV-treatment optimism subscale 1.91 ( $\mathrm{sd}=.77$; range $1-4$ ), the safe-sex necessity subscale 1.31 ( $\mathrm{sd}=.55$; range $1-3.3$ ), and the safe sex fatigue subscale 2.30 ( $\mathrm{sd}=1.03$; range 1-5). Mean number of lifetime same-sex partners was 5.4 ( $\mathrm{sd}=8.56$, median $=3$, modus $=1)$, and $48 \%$ reported four or more lifetime partners. Seventeen percent $(\mathrm{N}=31)$ of the respondents had ever tested for HIV. Finally, mean intention was 6.22 $(\mathrm{sd}=1.17)$, with $51 \%$ reporting a maximum intention.

Of the 185 respondents who completed the first questionnaire, $141(76 \%)$ completed all four questionnaires. Drop-out between wave one and wave four was significantly related to educational level; of respondents with a lower educational level 33\% dropped-out compared to $18 \%$ of respondents with a higher educational level $\left(\chi^{2}=5.23, \mathrm{p}<.05\right)$. No other variables (i.e. age at baseline, number of lifetime same-sex partners at baseline, HIV-treatment optimism at baseline (and its sub-scales), behavioral intention at baseline) were significantly related to drop-out (all p's $>.05$ ).

Table 4.1 shows the proportion of respondents who reported UAI in the six months preceding each wave. These proportions did not significantly change per wave $\left(\chi^{2}=8.68, \mathrm{df}=9, \mathrm{p}>.05\right)$.

Table 4.1 Proportion of YGBM practicing anal intercourse in four biannual waves of Outcomes.

\begin{tabular}{lcccc}
\hline & \multicolumn{4}{c}{ Biannual interval } \\
\cline { 2 - 5 } & $\begin{array}{c}\text { Wave 1 } \\
\text { (N=185) }\end{array}$ & $\begin{array}{c}\text { Wave 2 } \\
\text { (N=162) }\end{array}$ & $\begin{array}{c}\text { Wave 3 } \\
\text { (N=158) }\end{array}$ & $\begin{array}{c}\text { Wave 4 } \\
\text { (N=152) }\end{array}$ \\
\hline No intercourse & $21 \%$ & $21 \%$ & $18 \%$ & $14 \%$ \\
No anal intercourse & $28 \%$ & $24 \%$ & $27 \%$ & $24 \%$ \\
Protected anal intercourse & $15 \%$ & $21 \%$ & $18 \%$ & $26 \%$ \\
Unprotected anal intercourse & $36 \%$ & $34 \%$ & $37 \%$ & $37 \%$ \\
\hline
\end{tabular}


Longitudinal patterns of sexual risk behavior are presented in Table 4.2. Of the respondents who completed all four data waves, $41 \%$ never reported sexual risk behavior (i.e. no intercourse, no anal intercourse or protected anal intercourse), $45 \%$ reported variable sexual risk behavior, and $14 \%$ reported sexual risk behavior at all four data waves. Of the $45 \%$ who reported variable risk behavior, $12 \%$ changed from unsafe to safe sexual behavior and $17 \%$ changed from safe to unsafe.

Table 4.2 Longitudinal patterns of sexual risk behaviors in a cohort of YGBM in the Netherlands $\left(\mathrm{N}=141^{*}\right)$.

\begin{tabular}{|c|c|c|c|}
\hline Behavior category & Pattern ${ }^{* *}$ & $\%$ & $\mathbf{N}$ \\
\hline Consistent no risk & nnnn & $41 \%$ & 58 \\
\hline \multirow[t]{15}{*}{ Variable risk } & unnn & $4 \%$ & 5 \\
\hline & uunn & $4 \%$ & 6 \\
\hline & uuun & $4 \%$ & 6 \\
\hline & nnnu & $9 \%$ & 12 \\
\hline & nnuu & $4 \%$ & 6 \\
\hline & nuuu & $4 \%$ & 6 \\
\hline & nnun & $2 \%$ & 3 \\
\hline & nunn & $1 \%$ & 2 \\
\hline & nunu & $4 \%$ & 5 \\
\hline & nuun & $3 \%$ & 4 \\
\hline & unnu & $1 \%$ & 2 \\
\hline & unun & $2 \%$ & 3 \\
\hline & unuu & $3 \%$ & 4 \\
\hline & uunu & - & 0 \\
\hline & total & $45 \%$ & 64 \\
\hline Consistent risk & uuuu & $14 \%$ & 19 \\
\hline
\end{tabular}

"Only respondents who completed all four questionnaires are included.

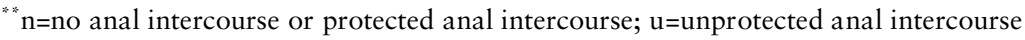


Table 4.3 shows univariate associations between risk group and demographics and behavioral and cognitive correlates measured at baseline ${ }^{9}$. As can be seen, the odds on reporting consistent risk behavior are higher for respondents who had four or more lifetime sexpartners, for respondents who ever tested for HIV, for respondents who report increased safe sex fatigue and for respondents who report increased HIV-optimism, compared to the odds on reporting no risk behavior. Also, the odds on reporting consistent risk behavior are lower for respondents with a maximum intention compared to respondents with a lower intention. Finally, the odds reporting variable risk behavior are higher for respondents reporting four or more lifetime same-sex partners, for respondents reporting increased safe sex fatigue, and for respondents reporting increased HIV-optimism compared to the odds on reporting consistent no risk behavior. Also, the odds on reporting variable risk behavior are lower for respondents with a maximum intention compared to respondents with a lower intention.

\footnotetext{
${ }^{9}$ In order to assess differences between the consistent risk group and the variable risk group, all multinomial logistic regression analyses were also performed with consistent risk group as reference category. All p's in these analyses were $>.05$, indicating that the odds on reporting consistent risk behavior did not differ from the odds on reporting variable risk behavior for any of the independent variables.
} 
Table 4.3 Univariate nominal logistic regression analysis with risk group as dependent variable ("consistent no risk" is the reference category) and demographics, behavioral and cognitive variables measured at wave 1 as covariates $(\mathbf{N}=141)$.

\begin{tabular}{|c|c|c|c|c|}
\hline \multirow[b]{2}{*}{ Covariate } & \multicolumn{2}{|c|}{ Variable risk group } & \multicolumn{2}{|c|}{ Consistent risk group } \\
\hline & OR & $95 \% \mathrm{Cl}$ & OR & $95 \% \mathrm{Cl}$ \\
\hline Age & .99 & $.84-1.17$ & 1.11 & $.89-1.38$ \\
\hline \multicolumn{5}{|c|}{ Educational level } \\
\hline Lower & 1.00 & & 1.00 & \\
\hline Higher & 1.00 & $.47-2.14$ & 1.08 & $.36-3.30$ \\
\hline \multicolumn{5}{|c|}{$\begin{array}{l}\text { Number of lifetime } \\
\text { same-sex partners }\end{array}$} \\
\hline$\leq 3$ & 1.00 & & 1.00 & \\
\hline$\geq 4$ & $3.17^{* * *}$ & $1.49-6.76$ & $2.92^{* *}$ & $1.00-8.94$ \\
\hline
\end{tabular}

HIV-test ever

\begin{tabular}{|c|c|c|c|}
\hline No & 1.00 & & 1.00 \\
\hline Yes & $3.12^{*}$ & $.94-10.28$ & $4.82^{* * * *}$ \\
\hline
\end{tabular}

HIV-optimism

$\begin{array}{lcccr}\begin{array}{l}\text { Treatment } \\ \text { optimism }\end{array} & 1.15 & .73-1.81 & .82 & .41-1.65 \\ \begin{array}{l}\text { Safe sex necessity } \\ \text { Safe sex fatigue }\end{array} & 1.24 & .64-2.42 & 1.46 & .61-3.53 \\ \begin{array}{l}\text { Total HIV } \\ \text { treatment } \\ \text { optimism scale }\end{array} & 1.64^{* *} & 1.12-2.40 & 2.24^{* * *} & 1.32-3.78 \\ & 1.80^{* *} & 1.01-3.24 & 2.20^{* *} & 1.01-4.82\end{array}$

Behavioral intention

\begin{tabular}{lrrrr} 
Lower & 1.00 & 1.00 & \\
High & $.44^{* *}$ & $.21-.92$ & $.20^{* * *}$ & $.06-.64$ \\
\hline $\mathrm{p}<.10,{ }^{* * *} \mathrm{p}<.05,{ }^{* * *} \mathrm{p}<.01$ & &
\end{tabular}

\section{Discussion}

The Outcomes cohort study shows that substantial numbers of YGBM have unprotected sex in each of four biannual data waves. Moreover, the proportion of YGBM engaging in UAI did not significantly change over a period of two years. The observed proportions of UAI per data 
wave (i.e. $34-37 \%$ ) are well in line with proportions observed in other cross-sectional surveys and studies, both among younger and older MSM [5, 6, 20, 46, 51, 52, 98]. The prospective nature of the Outcomes study enabled us to examine longitudinal patterns of sexual risk behaviors. These longitudinal behavior patterns show that a relatively small proportion of YGBM engaged in consistent risk behavior (i.e. $14 \%$ reported UAI at each data wave) and a larger proportion variably engaged in sexual risk behavior (i.e. $45 \%$ engaged in UAI at one or more data waves but not at all four data waves). A previous Dutch MSM cohort study, conducted more than 2 decades ago in an era in which relatively little was known about HIV risk in the Netherlands, showed that $29 \%$ reported consistent risk and $65 \%$ reported variable risk behavior [97]. Other more contemporary cohort studies conducted in the United States (e.g. [20, 99-103]), Australia (e.g. [61, 83, 104]) and Europe (e.g. [56, 82, 105]) do not report on patterns of longitudinal sexual behavior.

The Outcomes study also shows that the correlates of sexual risk behavior did not differ between the consistent and the variable risk group. However, both the consistent and the variable risk group significantly differed on a number of independent variables from the consistent no risk group. First, in concordance with other studies [20, $95,96]$, the behavioral intention to always use condoms when having anal sex measured at baseline was highly negatively associated with subsequent variable and consistent sexual risk behavior. From a practical point of view, interventions promoting consistent condom use should aim at increasing intention to always use condoms when having anal intercourse. According to cognitive-motivational theories, intentions are influenced through attitudes, social norms and perceived behavioral control ([25]; for specific methods, see [22, 96]).

Second, in contrast to other cross-sectional studies [91, 93, 94, 106], we observed a relationship between HIV-optimism at baseline and subsequent sexual risk behavior. YGBM who were consistently unsafe in each data wave were more optimistic about HIV at baseline compared to YGBM who were consistently safe. However, our measure of HIV-optimism consisted of three dimensions (i.e. treatment 
optimism, safe sex necessity and safe sex fatigue) and only the safe sex fatigue sub-scale was associated with longitudinal sexual risk behavior. Safe sex fatigue refers to feelings of indifference or apathy towards always practicing safe sex and YGBM who express these feelings are more likely to report consistent or variable risk behavior. Although a previous prospective study among MSM of all ages also found a positive relationship between safe sex fatigue and sexual risk behavior [107], our finding is surprising in that these YGBM are still early on in their sexual trajectory. Preventive interventions should also focus on influencing these feelings of safe sex indifference, for example through influencing their attitudes towards safer sex.

Third, the odds on reporting consistent sexual risk behavior, but not on variable risk behavior, were greater for respondents who ever have been tested for HIV. HIV-testing has been associated with increased sexual risk behavior [62, 88]. However, the causal relationship between HIV-testing and sexual risk behavior is not yet clear. In our study we observed a positive association between ever being tested for HIV and subsequent consistent sexual risk behaviors. However, this does not imply that HIV-testing facilitates UAI. More likely, other factors, such as risk perception or exposure to prevention messages mediate this relationship. The significant association between ever being tested for HIV and consistent sexual risk behavior could also point to missed opportunities for posttest counseling. However, we did not assess the content and the quality of the posttest counseling.

Finally, the odds on reporting consistent and variable risk behavior was greater for YGBM who report four or more lifetime same-sex partners compared to the consistent no risk group, a finding which is not uncommon in MSM research [46, 101, 108]. However, having more sex partners also increases the opportunity to engage in UAI. Moreover, any UAI is a relatively crude measure of actual risk in that some YGBM who report multiple sex partners may have engaged in one episode of UAI whereas others may have engaged in multiple episodes of UAI [104]. Also, it should be noted that the consistent no risk group in this study also consisted of respondents who did not engage in anal sex or who did not have sex, which may have inflated 
the observed odds ratios. Nevertheless, this finding may still provide valuable information for intervention development. For example, preventive messages may be tailored specifically towards YGBM who report multiple sex partners.

The Outcomes study has several limitations. First of all, the cohort consists of YGBM who were, at least at time of recruitment, in the midst of their coming-out. Although there is evidence that specific coming-out related factors influence the decision to engage in sexual risk behavior (e.g. involvement in gay-related activities, positive attitudes towards homosexuality and disclosure of sexual identity to more individuals [20,32]), in the present paper we did not explore these associations. Nevertheless, this warrants that our findings should be interpreted within the context of our sample. Second, whereas respondents were recruited both offline and online, data collection took place online. However, Internet surveys seem to produce as reliable results as telephone interviews or pencil and paper interviews [109-111]. Third, a six months interval between data waves may have been too short to observe a sufficient number of respondents performing the behavior under study (i.e. anal intercourse). The consistent risk group contained a limited number of respondents which may have resulted in a lack of statistical power, especially for comparisons between the consistent and variable risk group. A fourth limitation is that not all factors which have been found to be associated with sexual risk behaviors were assessed in the current study (e.g. illicit drug use prior or during sex [20], personality factors such as impulsivity and sexual sensation seeking [20,46], family and partner violence [78, 79], childhood sexual abuse [20]). Finally, the present analyses consisted only of respondents who were not lost to follow-up, and therefore our results may not fully represent the target population. However, respondents who dropped-out from the study did not significantly differ on critical independent variables used in the present analyses.

The Outcomes study is one of the first studies reporting longitudinal sexual risk behaviors of YGBM. The results showed that a considerable proportion of YGBM engage in sexual risk behavior, and 
these proportions did not change over time. Furthermore, this study showed that the largest risk group consisted of YGBM who variably engaged in sexual risk behavior (i.e. any combination of no risk and UAI over four data waves), and that a smaller group consistently engaged in sexual risk behavior. HIV-prevention activities must also focus on increasing intention to always use condoms and reducing feelings of safe sex apathy. 



\section{Chapter 5 Social-cognitive determinants of condom use in a sample of young gay and bisexual $\operatorname{men}^{10}$}

According to UNAIDS approximately 33.2 million people were living with HIV/AIDS in the world by the end of 2007 [1]. The majority of HIV-infections occur through sexual contact, and men who have sex with men (MSM) represent the largest proportion of HIV-diagnoses in most Western counties [1]. Recently it has been suggested that young gay and bisexual men (YGBM) are at elevated risk of contracting HIV and other STIs through unprotected anal intercourse (UAI, [46, 48, 49, $54,55])$. A thorough understanding of the reasons why YGBM are not using condoms when having anal sex can facilitate the design of effective interventions to increase condom use among YGBM [22, 86]. The aim of this study is to examine social-cognitive determinants of condom use with casual same-sex partners in a sample of YGBM.

In order to design effective HIV-prevention interventions for this target group, social-cognitive determinants of condom use have to be known [22]. The theory of planned behavior (TPB, [25]) is a useful model for predicting and understanding determinants of intention and behavior [112-114]. Moreover, interventions based on determinants from social-cognitive theories such as the TPB have been shown to be effective in changing various health behaviors $[95,115]$. In short, the theory states that behavior is best predicted by behavioral intentions and that people form these behavioral intentions based on their attitudes, subjective norms, and perceived behavioral control. The TPB

\footnotetext{
${ }^{10}$ This chapter is co-authored by Harm Hospers and Gerjo Kok and has been published in AidsCare, volume 21, pages 1471-1479, under the same title.
} 
also assumes that other more distant variables, such as demographics, influence intention and behavior through these three determinants. Research has shown that the predictive value of the TPB can be further increased by adding constructs to the model, such as descriptive norms [116], personal norms [117-119], and behavioral willingness [75, 76].

The TPB has been shown to predict intentions to use condoms and prospective condom use behavior reasonably well in a variety of samples (for reviews see [95, 120], including MSM [121-124]. For example, two recent Dutch MSM studies showed that the three main TPB constructs were all significantly related to behavioral intention and adding descriptive and personal norms increased the explanatory power of the model $[57,123]$. Moreover, a substantial correlation $(\mathrm{r}=0.58 ; \mathrm{P}<.001)$ between intention and actual behavior was found [57].

The Outcomes project is an online cohort study on coming-out and sexual behavior. Between early 2007 and late 2009 participants complete six biannual questionnaires. One aim of this project is to investigate the social-cognitive determinants of condom use with casual partners in a sample of YGBM who are in the midst of their comingout. TPB variables regarding condom use with a casual partner were assessed during the first wave of the study, while sexual behavior with casual partners was assessed at the second wave six months later.

\section{Methods}

The Outcomes study is an online cohort study on coming-out and sexual behavior. Between early 2007 and late 2009, respondents complete six biannual questionnaires. The study was approved by the ethics committee of the Faculty of Psychology and Neuroscience, Maastricht University. 


\section{Recruitment}

The eligibility criteria were being between 16 and 25 years of age ${ }^{11}$, being sexually attracted to men, and first disclosure of sexual attraction in the year prior to the survey. In the present study disclosure was defined as telling at least one significant other (e.g. parents, siblings, friends or teacher at school) about attraction to the same sex. It should be noted that by using this working definition, we excluded disclosing sexual identity anonymously (e.g. through online chatting in gay chat sites or through anonymous participation in gay-related Internet forums).

Cohort participants were recruited both offline $(45.9 \%)$ and online $(54.1 \%)$. Offline recruitment took place at various gay venues frequented by YGBM. Details of YGBM (i.e. age, recency of comingout and telephone number) were obtained and eligible participants were contacted by telephone within three working days. Other offline recruitment consisted of placing advertisements promoting the web address of the survey in gay-related media aimed at YGBM. Online recruitment consisted of placing banners with a link to the survey website on various gay-related sites frequented by YGBM and by issuing a press release which was published on various gay news sites. On the website of our survey, interested participants could submit their contact details (e-mail and telephone number) and if they did they were contacted by the primary researcher within three working days. The telephone interview was primarily aimed at verifying inclusion criteria and to clarify the procedure of the study. Eligibility was confirmed by asking interested participants' age, their sexual attraction $(1=$ only attracted to men and 5= only attracted to women) and when and to whom they first disclosed their sexual attraction. Next, to ensure that only the intended participants entered the cohort, eligible participants received an e-mail with a personal login name and password which they had to use to login to the website that contained the questionnaire. The telephone interview also provided us with the

\footnotetext{
${ }^{11}$ Although some gay and bisexual youth in the Netherlands nowadays come out before the age of 16 , it was deemed inappropriate to include them in this study since that would have required parental consent.
} 
opportunity to verify whether intended participants were trying to misrepresent eligibility and to strengthen the commitment of the participants for participation in the cohort study.

A total of 185 participants, who were in the midst of their coming-out (median number of months after coming-out: 7; interquartile range: 4-10) completed their first online questionnaire on coming-out milestones and sexual behavior.

\section{Questionnaire}

The first part of the first online questionnaire consisted of demographics (e.g. age, educational status, cultural background). The second part consisted of questions on sexual history (e.g. first same-sex partner) and questions on recent sexual behavior and condom use in the six months preceding the survey for steady, regular and casual partners. The third part contained the social-cognitive determinants of condom use with a new partner in the next six months. The various beliefs were derived from the literature and from previous research conducted in the Netherlands [57, 125].

Behavior was assessed by asking respondents whether they had receptive or insertive anal intercourse with a casual partner and whether they used condoms ( 1 =never; $5=$ always; separate for receptive and insertive anal intercourse). Behavior was coded as safe when a condom was used on all episodes of both receptive and insertive anal intercourse and unsafe when at least one episode of unprotected receptive or insertive anal intercourse was reported.

Attitude was measured by averaging 11 attitudinal belief statements about condom use with a new partner in the next six months. For example, "using condoms when having anal sex with a new partner reminds too much of HIV/AIDS". Responses were on a five point scale, ranging from completely disagree to completely agree. Six negative items were recoded so that a higher score indicates a more positive attitude towards using condoms. A reliability analysis showed that one belief (i.e. "condoms are expensive") did not scale and was therefore deleted from the scale. Because this belief was not significantly correlated with intention $(r=.028, p>.05)$ or with behavior 
$(\mathrm{r}=-.16, \mathrm{p}>.05)$, it was not incorporated in subsequent analyses. Cronbach's alpha of the final scale is .72 .

Subjective norm was measured by three normative beliefs (social referents were homosexual friends, heterosexual friends, and parents). For example: "My homosexual friends believe that I should always use condoms when having anal sex with a new partner". Responses were on a five point scale ranging from most certainly not to most certainly, plus "don't know" and "not applicable" (both coded as middle score). Alpha of the scale is .70.

Perceived behavioral control was measured by beliefs about confidence to use condoms in five situations (i.e. when in love, when physically attracted, when excited, when consumed large amount of alcohol, and when under the influence of drugs). Moreover, three beliefs about the respondents' confidence to tell a new partner that he wants to use condoms was measured (i.e. tell in general, when partner pressures you to have insertive anal intercourse without a condom or receptive anal intercourse without a condom). Finally, three beliefs about the respondents' confidence to use a condom with a new partner were measured (i.e. buy condoms, putting a condom on to yourself, and putting a condom on to a new partner). Example: "Are you confident that you are able to always use a condom when having anal sex with a new partner with whom you are in love?". Responses were on a five point scale ranging from most certainly not to most certainly. Alpha of the scale is .87.

Descriptive norm was measured by two beliefs on the respondents' perception of condom use of their homosexual friends and of YMSM in general. For example: "Imagine your homosexual friends are having sex with a new partner, do you think they always use condoms for anal sex?". Responses were on a five point scale ranging from most certainly not to most certainly, plus "don't know" and "not applicable" (both coded as middle score). The two beliefs were significantly correlated $(\mathrm{r}=.56, \mathrm{p}<.01)$. Therefore, they were summed to form a general descriptive norm measure.

Personal norm was measured by four beliefs about condoms: for me it is normal, it would be against my principles, I would feel guilty, 
and I feel morally obliged to. For example: "I for me do think it is normal to use condoms when having anal sex with a new partner in the next six months". Responses were on a five point scale ranging from completely disagree to completely agree. Alpha of the scale is .80 .

Behavioral willingness was measured by five beliefs about willingness to have unprotected sex with a new partner in the next 6 months: when he says he is HIV-negative, when you think he is HIVnegative, when there are no condoms present, when you think he is going to be your steady partner of when you think he is going to be you regular partner. For example: "If you have sex with a new partner in the next 6 months, are you willing to have unprotected sex when you think he is HIV-negative?" Response were on a seven point scale, ranging from most certainly not tot most certainly so. Alpha of the scale is .93 .

Behavioral intention was measured by two questions, I intend to and I expect to. For example: "Imagine you have anal sex with a new partner in the next six months. Do you intend to use a condom?”. Responses were on a seven point scale ranging from most certainly not to most certainly. The two items were highly correlated $(\mathrm{r}=.84, \mathrm{p}<$ .01 ) and therefore summed to form a general intention measure.

The final part of the online questionnaire included questions about gay community attachment, mental health concerns and discrimination, which will be reported elsewhere. The follow-up questionnaire essentially consisted of the same topics and thus similar questions as the first questionnaire and will not be described in detail here. Participants receive $€ 10$ for completing each questionnaire.

\section{Data analyses}

Descriptive statistics about demographics and UAI with casual partner were obtained. Because only four respondents $(2 \%)$ had a non-western cultural background, the analyses are restricted to respondents with a Dutch or western cultural background ( $\mathrm{N}=181)$. Next, correlations between the TPB constructs and intention (Pearson's correlation) and behavior (point biserial correlation) were obtained. Next, hierarchical linear regression analysis was used to examine relationships between determinants and intention. In the first step the three main 
determinants of intention were entered. In the second step the two extra determinants (i.e. descriptive and personal norms) were added and in the third step demographics were added. Next, three separate logistic regression analyses were performed to predict UAI with casual partners from intention, perceived behavioral control and behavioral willingness. Finally, a series of Students' T-Test were performed to investigate differences in belief structure between high versus lower intenders and between safe versus unsafe sexual behavior with casual partners ${ }^{12}$. A significance level of .05 was used in all analysis, except in the analyses for the differences in beliefs, where a p-value of .01 was used to correct for multiple testing.

\section{Results}

\section{Sample characteristics}

Participants' mean age at wave 1 was 18.9 ( $\mathrm{sd}=2.3$; median: 18, range 16-26). The vast majority had a Dutch cultural background (98\%). Forty percent of the respondents $(\mathrm{N}=63)$ reported having had sex with casual partners in the six months between wave 1 and wave 2, of whom $32(51 \%)$ had no anal intercourse. Of the YGBM who had anal sex with casual partner(s), $18(58 \%)$ had protected anal intercourse and $13(42 \%)$ had at least one episode of unprotected receptive or insertive anal intercourse. Of all the YGBM who reported sex with a casual partner, $21 \%$ reported at least one episode of UAI.

\section{Correlations}

First of all, demographic characteristics were not significantly correlated with intention and behavior (see Table 5.1). Only educational level was significantly positively correlated with attitudes

\footnotetext{
${ }^{12}$ Initially, the relation between intention measured at wave 1 and behavior measured at wave 2 was analyzed for new partners because all social-cognitive determinants were assessed for anal sex with new partners. A subsequent explorative analysis of the relation between intention at wave 1 and behavior with steady, casual and regular partners at wave 2 revealed a significant relation between intention and behavior only for casual partners. Because the amount of explained variance in behavior was $28 \%$ for casual partners and $11 \%$ for new partners, only the results for casual partners will be described in detail.
} 
and negatively correlated with behavioral willingness; no other demographic characteristics were significantly correlated with any of the TPB or other social-cognitive variables. Second, there was a significant correlation between intention and behavior $(\mathrm{r}=.40 ; \mathrm{p}<.05)$, indicating that respondents who reported UAI with a casual partner had a lower intention to use condoms. No other variables significantly correlated with behavior. Finally, the variables most strongly correlated to intention were: attitudinal beliefs, personal norms, descriptive norms and perceived control (see Table 5.1).

\section{Prediction of behavior and intention from social- cognitive variables}

First of all, a stepwise backward logistic regression-analysis with dropout ( $1=$ dropout; $2=$ non-dropout) as dependent variable and background variables (age, and educational level), TPB variables and behavior as independent variables showed that respondents who did not dropout at wave 2 perceived a higher social norm to always use condoms with a new partner $(\mathrm{M}=4.06)$ than respondents who dropped out $(\mathrm{M}=3.70$; Adjusted $\mathrm{OR}=1.88,95 \%$ CI 1.05 - 3.36). No other variables significantly differed between dropouts and non-dropouts.

Next, a hierarchical linear regression analyses showed that a model with three main TPB variables explained $28 \%$ of the variance in intention (see Table 5.2). In this model, only the variables attitudes and perceived behavioral control were significant ${ }^{13}$. Adding descriptive and personal norm to the model increased the percentage of explained variance by $8 \%$. Finally, adding demographics did not significantly increase the percentage of explained variance. Thus, in the final model, attitudes, descriptive norm, perceived behavioral control, and personal norms significantly predict intention.

\footnotetext{
${ }^{13}$ Because a substantial number of respondents indicated "Don't know" and "Not applicable" for the subjective norm items (see also Table 3), we also coded "Don't know" and "Not applicable" as missing. For the sub-group of respondents who do know what their social referents think they should do, subjective norm does significantly predict intention (beta $=.19 ; \mathrm{p}=.004$ ).
} 


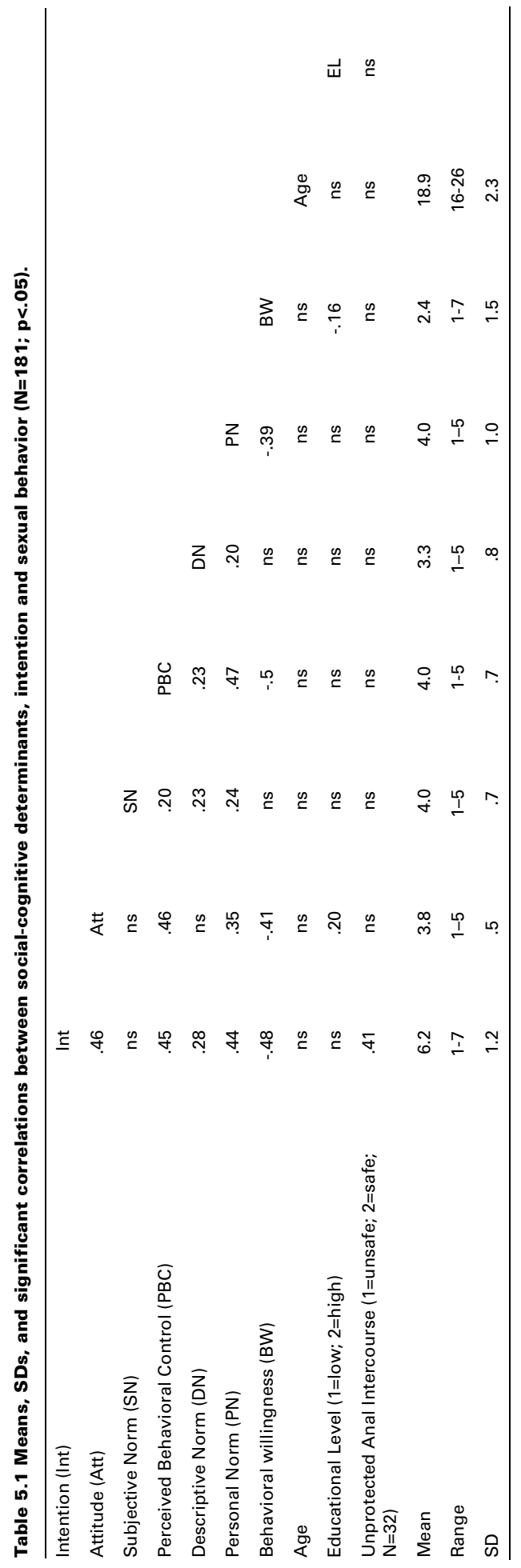


Table 5.2 Prediction of condom use intention from social cognitive variables (all reported standardized beta's are significant $p<.05$ ).

\begin{tabular}{lccc}
\hline Variable & Model $\mathbf{\beta}$ & Model $\mathbf{\beta}$ & Model 3 $\boldsymbol{\beta}$ \\
\hline Theory of planned behavior & .32 & .27 & .26 \\
$\quad$ Attitude & $\mathrm{ns}$ & $\mathrm{ns}$ & $\mathrm{ns}$ \\
$\quad$ Subjective norm & .31 & .19 & .18 \\
$\quad$ Perceived behavioral control & .28 & & \\
$\mathrm{R}^{2}$ & & & \\
Other psychosocial variables & & .16 & .16 \\
$\quad$ Descriptive norm & & .24 & .23 \\
$\quad$ Personal norm & & .36 & \\
$\mathrm{R}^{2}$ & & & $\mathrm{~ns}$ \\
Demographic variables & & & $\mathrm{ns}$ \\
$\quad$ Age & & & .37 \\
$\quad$ Educational status & & & \\
$\mathrm{R}^{2}$ & & &
\end{tabular}

Finally, a logistic regression analysis showed that intention significantly predicted condom use (adjusted OR=5.00; 95\% CI $1.14-21.85$; model fit compared to model with only constant: block $-2 \mathrm{LL}=7.49$; $\mathrm{p}<.01)$. Both perceived behavioral control and behavioral willingness did not significantly predict behavior ( $p>.05$ ). Thus, of the respondents who reported a maximal intention to always use condoms, $77 \%$ always used a condom in the six months between wave 1 and wave 2, and of the respondents who reported a less than maximal intention, $36 \%$ always used a condom.

\section{Differences between beliefs}

First of all, because intention to use condoms with a casual partner was very positive and because $51 \%$ of the respondents had a maximal intention, intention was dichotomized by categorizing 'most certainly' as high intention and all other responses as lower intentions. In general, respondents had a positive attitude towards condom use with a new partner (see Table 5.3). The negative consequences of condom use (i.e. irritating disturbance, creates distrust, less intimate) are not salient for high intenders and somewhat salient for lower intenders. 
The positive aspects (i.e. hygienic and feeling of being safe) of condom use were more important for high intenders. The subjective norm does not differentiate between high and low intenders. Only the descriptive norm what their best friends do when having sex with a new partner was more salient for high intenders. High intenders held strong moral norms regarding condom use with a new partner. They belief that it is normal to use condoms and that they would feel guilty if they did not use condoms. Moreover, they believe it is against their principles not to use condoms and they feel morally obliged to use condoms when having sex with a new partner. Finally, confidence to use condoms when having anal sex with a new partner was very high. High intenders felt more confident to use condoms with a new partner when they are in love, when the partner is attractive, or when they are sexually aroused. Also, high intenders expressed more confidence to be able to tell their new partner that they want to use condoms in general and in two specific situations (i.e. when partner wants unprotected insertive or receptive anal sex). High intenders also expressed more confidence to be able to buy and carry condoms and they felt more capable of putting on a condom on oneself and on the partner. 
Table 5.3 Differences in means between respondents with a high or lower intention to use condoms when having anal sex with casual partner(s) $(\mathbf{N}=181)$. All scores range between 1-5 expect for behavioral willingness which scores range between 1-7. A higher score indicates more agreement with each belief. For attitudinal beliefs, negative items were recoded so that a higher score indicates a more positive attitude. As a result, items 1, 3, 4, 5, 6 should be read as follows: a higher score indicates less agreement.

Belief

\section{ATTITUDINAL BELIEFS}

Is an irritating disturbance

Is extra exciting

Less feeling

Creates distrust

Reminds too much of HIV

Is less intimate

Is hygienic

Creates feeling of being safe

Good protection against HIV

Good protection against other STIs

\section{SUBJECTIVE NORMS}

My gay friends think that I should use condoms (40\% checked option "don't know or not applicable")

My straight friends think that I should use condoms (43\% checked option "don't know or not applicable")

My parents think that I should use condoms (31\% checked option "don't know or not applicable")

\section{PERCEIVED CONTROL}

Confident, when in love

Confident, when partner is attractive

Confident, when sexually aroused

Confident, when drink too much alcohol

Confident, when use drugs

Confident about telling partner to use condoms

Idem, if partner does not want to for insertive anal sex

Idem, if partner does not want to for receptive anal sex
High intention

Lower intention

$\begin{array}{ll}3.76 & 3.05^{* *} \\ 2.14 & 2.04 \\ 3.24 & 2.82 \\ 4.34 & 3.83^{*} \\ 4.16 & 3.83 \\ 3.88 & 3.26^{*} \\ 4.50 & 4.04^{*} \\ 4.69 & 4.27^{* *} \\ 4.68 & 4.42 \\ 4.53 & 4.46\end{array}$

3.96

4.16

$3.32^{* *}$

4.40 $3.68^{* *}$

4.06 $3.29^{* *}$

3.51

3.15

3.49

3.10

4.83

$4.33^{* *}$

4.71

$4.08^{* *}$

4.73

$3.92^{* *}$ 


\section{Belief}

Confident, always carry condoms

Confident, buy condoms

Confident, apply condoms to self

Confident, apply condom to new partner

DESCRIPTIVE NORM

My gay friends use condoms

YMSM use condoms

PERSONAL NORM

For me, it's normal to use condoms

I feel guilty if I do not use condoms

It is against my principles not to use condoms

I feel morally obliged to use condoms

BEHAVIORAL WILLINGNESS

Willing, when new partner says he is HIV-negative

Willing, when you think new partner is HIV-

negative

When no condoms are present

When you expect him to become your regular partner

When you expect him to become your steady partner

\section{High intention}

3.88

4.29

4.74

4.55

3.66

3.20

4.73

4.30

4.46

4.20

1.78

1.58

1.79

1.76

2.06
Lower

intention

$3.19^{* *}$

3.85

$4.28^{* *}$

$4.08^{*}$

$3.31^{*}$

3.06

4. $10^{* *}$

$3.23^{* *}$

$3.51^{* *}$

$3.56^{*}$

$3.27^{* *}$

$2.77^{* *}$

$3.23^{* *}$

$3.03^{* *}$

$3.87^{* *}$

\$ For scaling, the negative items were recoded

" $\mathrm{p}<.05, "$ p $<.01,{ }^{, * * *} \mathrm{p}<.001$

\section{Discussion}

The Outcomes study aimed to establish the relevant social-cognitive determinants of intention and actual condom use with casual partners. First of all, of the YGBM who reported sex with a casual partner in the six months between wave 1 and wave 2 , about one fifth reported at least one episode of unprotected anal sex. Second, the TPB was successful in identifying the relevant determinants of intention to use condoms when having anal sex with a casual partner. The final model, which best predicted intention to use condoms, consisted of attitudes, perceived control, personal norms and descriptive norms. Moreover, 
condom use with casual partners at six months follow-up was significantly predicted by intention to use condoms.

The Outcomes study showed that for YGBM, social-cognitive factors play a role in the decision to use condoms when having anal sex with a casual partner. Our findings are well in line with previous TPB studies although the level of explained variance is somewhat lower compared to studies of older MSM [57, 121, 123, 124, 126].

In line with the TPB, we found that behavior at six-month follow-up is best predicted by behavioral intention. Intention explained $28 \%$ of the variance in subsequent behavior, a finding which corroborates previous research (see for an overview of reviews [127]. Moreover, the vast majority of the YGBM who expressed a maximal intention to use condoms subsequently acted upon their intention. These results suggest that intention plays an important role in the decision to engage in protected anal sex with casual partner(s).

In general, YGBM hold favorable attitudes towards condom use with a new partner. Whereas YGBM with a lower intention see more disadvantages of condom use (e.g. irritating disturbance, creates distrust), high intenders see more advantages of condoms (e.g. is hygienic and creates feelings of being safe). High intenders are also very confident that they can buy, carry, and use condoms. Moreover, they are confident that they can tell a new partner that they want to use condoms, even in difficult situations such as when they are sexually aroused or when the partner does not want to use condoms. Social influences do not differentiate between high and lower intenders, expect for the descriptive belief that their best gay friends always use condoms when having sex with a new partner. Finally, YGBM with a high intention express more personal responsibility to use condoms. They feel that it is against there principle not to use condoms and they would feel guilty if they do not use condoms when having anal sex.

From a practical point of view, the Outcomes study suggests that HIV-preventive interventions directed at YGBM should focus on attitudes, personal norms, descriptive norms and perceived behavioral control. Furthermore, this study also provided information on which specific beliefs are more relevant in explaining condom use and as such 
should be targeted in an intervention. YGBM should perceive that condom use has advantages related to HIV and STI prevention but should also anticipate disadvantages of condoms use (e.g. less intimate). Methods for changing attitudes are for example persuasive communication (for an overview, see [128]). Furthermore, YGBM should express confidence in buying and carrying condoms and also in negotiating condom use with a casual partner. Also, YGBM should express confidence in applying condoms to oneself and to a casual partner, even under difficult circumstances. Methods for increasing perceived behavioral control are skills training and modeling [27, 129]. Finally, the intervention should also try to establish, increase or reinforce personal norms towards condom use with casual partners.

The Outcomes study has several limitations. First of all, because the Netherlands is known to be a fairly liberal country with respect to homosexuality [77], some YGBM may have their coming-out before 16 years of age. Therefore, our results should be interpreted within the context of our sample. Second, because most respondents were recruited at gay Internet sites or at gay venues, it is possible that this study attracted YGBM who are already comfortable with their sexual identity which limits generalisability. However, there is no evidence that YGBM who visit gay related Internet sites or who frequent gay venues are also more comfortable with their sexual identity. In contrast, it is likely that YGBM nowadays use the Internet to search for information about sexuality and coming-out [34]. Finally, the Outcomes study collected the data online. However, Internet surveys seem to produce as reliable results as telephone interviews or pencil and paper interviews (e.g. [109-111]).

In conclusion, the Outcomes study investigated social-cognitive determinants of YGBMs condom use with a casual partner. Attitudes, perceived control, personal norms and descriptive norms all significantly predict intention to use condoms, and intention in turn predicted actual condom use. Furthermore, the Outcomes study provided information on which beliefs should be changed in an HIVprevention intervention. The next step in the development of interventions is to select theoretical methods and practical strategies to 
accomplish the desired changes in the determinants of condoms use with casual partners and to design and implement the actual intervention program. 


\section{Chapter 6 Minority-stress and depressive symptoms in a sample of young gay and bisexual men ${ }^{14}$}

There is ample evidence that young gay and bisexual men (YGBM) are at increased risk of emotional distress (i.e. depressive and anxiety symptoms) compared to their heterosexual counterparts $[28,32,90$, 130-137]. This elevated risk of emotional distress has been attributed to the experience of a unique set of stressors specifically related to belonging to a sexual minority $[2,130,135,138]$. YGBM in the midst of their coming-out may be particularly susceptible to experiencing these stressors $[32,133]$. Recently, a conceptual framework has been proposed which explains the higher prevalence of mental health problems observed in YGBM in terms of minority-stress [2]. This framework suggests that, next to general life stressors, specific minority-related stressors may induce feelings of emotional distress [2].The aim of the present study is to examine the relationship between minority-stress factors based on this conceptual minority-stress framework, and emotional distress, using data from a cohort of YGBM who were - at study entry - in the midst of their coming-out.

Several coming-out models have been proposed [11, 12, 14, 15, 19]. In general, these models describe coming-out as a process of identity formation and identity integration. In short, identity formation involves exploring the new sexual identity (e.g. involvement in gay related activities) and addressing possible negative attitudes towards being gay (i.e. internalized homonegativity). Identity integration involves accepting the new sexual identity (i.e. self-acceptance) and disclosing this sexual identity to others (i.e. disclosure). Furthermore,

\footnotetext{
${ }^{14}$ This chapter is co-authored by Harm Hospers and Gerjo Kok and a modified version
} of this chapter has been submitted for publication under the same title. 
most coming-out models assume that going through the coming-out process is characterized by increased feelings of emotional distress [12, 32, 139]. For example, fear of rejection because of non-heterosexual identity may cause YGBM to conceal their sexual identity for family and friends, and identity concealment in turn may result in stress [140, 141]. Also, internalized homonegativity, which is assumed to be especially prevalent in the early stages of the coming-out process [142], can negatively influence mental health $[139,143]$. In a similar vein, lower self-acceptance of sexual identity has been found to be associated with increased feelings of emotional distress [144]. Limited involvement in gay activities or limited connectedness with the gay scene may also contribute to feelings of emotional distress [32]. Finally, as YGBM progress in their coming-out, they may become more visible as gay and therefore risk facing acts of discrimination because of their sexual orientation. It has been shown that experienced discrimination can negatively impact mental health $[2,145,146]$.

Meyer [2] recently proposed a conceptual framework which captures the relationships outlined above between minority-related stress factors and emotional distress. The minority-stress framework describes two sources of stress: general stressors (e.g. stress from daily hassles) and minority-related stressors. The latter refer to both distal factors such as experienced acts of discrimination and to proximal factors such as having negative attitudes towards homosexuality or identity concealment (i.e. disclosure). The minority-stress framework also suggests that some factors may ameliorate stress and hence reduce emotional distress. For example, socializing with likeminded persons may enhance self-identity and hence may induce more positive attitudes towards homosexuality [32, 147].

The present study aims to examine the relationships between minority-stress factors and emotional distress, using data from an online longitudinal sample of YGBM who are in the midst of their coming-out. Although coming-out is generally considered to be a process $[11,12,15,19]$, in the present study coming-out was defined as the singular act of disclosing sexual identity face-to-face to at least one significant other within the preceding year. This enabled us to set 
clear recruitment criteria. Moreover, it ensured that most respondents were still in the process of disclosing their sexual identity to others. Finally, despite this rather strict inclusion criterion, we managed to include 185 respondents in the present study. In line with the minoritystress model, it is hypothesized that one distal stressor (i.e. experienced acts of discrimination because of sexual identity) and four proximal factors (i.e. self-label as gay or bisexual, self-acceptance of sexual identity, negative attitudes towards homosexuality and concealment of sexual identity) are associated with emotional distress. Furthermore, it is hypothesized that connectedness with the gay scene moderates the relationship between minority-stress factors and emotional distress.

\section{Methods}

The Outcomes study is an online cohort study on coming-out and sexual behavior. Between early 2007 and late 2009 participants complete six bi-annual questionnaires. The study was approved by the ethics committee of the Faculty of Psychology and Neuroscience, Maastricht University. The methods of the Outcomes study have been described in detail in previous chapters.

Cohort participants were recruited both offline $(45.9 \%)$ and online $(54.1 \%)$. Offline recruitment took place at various gay venues frequented by young MSM and by placing advertisements promoting the survey website in gay-related media aimed at young MSM. Online recruitment consisted of placing banners with a link to the survey website on various gay news sites frequented by young MSM. The first online questionnaire on coming-out and sexual behaviour was completed by 185 respondents.

\section{Measures}

The demographic characteristics of respondents were assessed using a fill-in-the-blank measure of age, postal code of place of residence (dichotomized into rural versus urban), an educational status checklist (dichotomized into lower versus higher education based on the Dutch educational system), and a cultural background checklist (dichotomized into Dutch or Western versus Non-Western). 


\section{Dependent variables}

Emotional distress was operationalised in the current study as experiencing depressive symptoms. This measure was derived from the Dutch version of the short Brief Symptom Inventory ([148]; for Dutch translation and psychometric properties see [149]). The Brief Symptom Inventory - Depression scale (BSIDEP) consists of 6 items and asks respondents to indicate whether each of six depressive symptoms occurred in the last week (i.e. week prior to completing the questionnaire). An example question is "How much did feelings of loneliness troubled you in the last week?". Responses were on a five point scale ranging from not at all to very much so. A higher score indicates increased feelings of depression. Alpha of the scale is .88. Mean scores (sd), median and mode are presented in Table 1. Dutch norm scores for the BSI-depression for YGBM are not available. However, the mean score of Dutch psychiatric patients is 1.58 ( $\mathrm{sd}=.55$; [149]) and this mean score will be used to classify respondents with normal (i.e. mean score $\leq 1.58$ ) or increased (i.e. mean score $>1.58$ ) feelings of depression.

\section{Independent variables}

Experienced discriminatory acts (discrimination) was measured by asking respondents to indicate if they experienced, in the six months preceding each questionnaire, any of the following discriminatory acts because of their sexual orientation: being called names, being bullied, being spit at, being hit, or being threatened. These items were tailored in such a way that respondents attending school received the question with the location being school and respondents holding a job with location being workplace. An example question is: "Have you experienced being called names at [school/work] because of your sexual orientation in the previous six months? All respondents had to indicate whether they experienced any of the discriminatory acts by strangers as well. Responses were on a five point scale, ranging from never to very often. Responses were collapsed across acts and subsequently dichotomized by classifying the response options "sometimes", "often" and "very often" as having experienced at least 
one act of discrimination versus having experienced no acts of discrimination.

Self-label of sexual identity (self-labeling) was assessed by asking respondents to indicate whether they perceive themselves as homo- or bisexual. Responses were coded as $1=$ homosexual and $2=$ bisexual.

Self-acceptance of sexual identity (self-acceptance) was assessed by asking respondents to indicate to what extent they accept their sexual identity. Responses were measured using a Visual Analogue Scale ranging from completely not accepting sexual identity $(0)$ to completely accepting sexual identity (100). A higher score indicates accepting sexual identity to a greater extent.

Negative attitudes towards homosexuality was conceptualized as internalized homonegativity (IHN), which was measured with 12 items derived from the Short Internalized Homonegativity Scale [150]. The original scale measures three aspects of homonegativity (i.e. Public identification as being gay, Sexual comfort with gay men and Social comfort with gay men). Two items (i.e. "it is important to me to control who knows about my homosexuality" and "making advances to another men is difficult for me") were removed from the original scale because these items did not scale. The final scale consists of 10 items measured on a five point scale ranging from completely disagree to completely agree. Alpha of the final scale is 0.66 . All items were recoded so that a higher score indicates more internalized homonegativity (i.e. more negative attitude towards homosexuality).

Concealment of sexual identity was conceptualized as level of disclosure (disclosure). Respondents were asked to indicate to what extent they consider themselves as “being out". Responses were measured using a Visual Analogue Scale ranging from completely not out (0) to completely out (100). A higher score indicates being more out about sexual identity.

Connectedness to the gay scene (connectedness) was measured by one item, 'To what extent do you feel connected to the gay-scene?'. Responses were measured using a Visual Analogue Scale ranging from completely not connected (0) to completely connected (100) to the gayscene. A higher score indicates more connectedness with the gay-scene. 


\section{Data analysis}

The aim of the present study is to explore associations between minority-related stressors and emotional distress. Descriptive statistics on demographics, minority-related stressors, and depression will be obtained. Next, as a preliminary test of associations between demographics, minority-related stressors and emotional distress, Pearson's and point-biserial correlations will be obtained. Next, the moderating effect of connectedness with the gay scene will be tested using five separate linear regression analyses (i.e. one for each minority-stress predictor). For the continuous predictors (i.e. selfacceptance, internalized homonegativity and disclosure), the interaction terms will be mean centered to reduce problems of multicollinearity [151]. A moderator role of connectedness would be supported if the interaction term is significant while controlling for the main effects of connectedness and the specific minority-related stress factor. To control for the influence of other factors (i.e. demographics and the minority-stress factors), a separate stepwise regression-analysis will be performed. Finally, as suggested by Preacher, Curran and Bauer [152], the nature of a significant interaction will be explored by testing the effect of the specific minority-stress factor on depression by three levels of connectedness (mean, 1 sd above the mean, and 1 sd below the mean; see also [153]), corrected for the influence of other mean centered covariates (see [152]). These analyses will be performed using the MLR two-way interaction tool described in Preacher, Curran and Bauer [152], which is available at www.quantpsy.org.

\section{Results}

Respondents' mean age was 18.8 (sd 2.3; median 18; range 16-26). The vast majority $(98 \%)$ had a Dutch or Western cultural background $^{15}$ and almost half of the respondents lived in urban areas

\footnotetext{
${ }^{15}$ Cultural background was significantly correlated with a number of study variables (e.g. depression, anxiety, self-acceptance, etc.). Because the Outcomes sample contained only four respondents with a non-Dutch or non-Western cultural background, and given that cultural background is correlated with a number of study variables, it was decided not to include these four respondents in subsequent analyses.
} 
(48\%). Seventy-nine percent of respondents are still attending school, and $62 \%$ of respondents attended or completed higher education at wave 1. Eighty-five percent of the respondents perceived themselves as being homosexual and $15 \%$ as being bisexual. Fifty-three respondents (i.e. 29\%) experienced at least one discriminatory act in the preceding six months. Finally, the mean score on the BSI-depression scale was 1.1 $(\mathrm{sd}=.94)$. Table 6.1 presents mean scores (sd) or percentages and correlations (Pearson's and point-biserial) on all study variables.

Several significant correlations emerged between demographics, minority-related stress factors and depression. First, significant correlations were observed between depression and age $(\mathrm{r}=-.20, \mathrm{p}<.05)$, educational level $(\mathrm{r}=-.17, \mathrm{p}<.05)$, self-acceptance $(\mathrm{r}=-.14, \mathrm{p}<.05)$, negative attitudes towards homosexuality $(\mathrm{r}=.14, \mathrm{p}<.05)$ and discrimination $(\mathrm{r}=.32, \mathrm{p}<.01)$.

Table 6.1 Means (sd) or frequencies and correlations between all study variables $(\mathbf{N}=185)$.

\begin{tabular}{|c|c|c|c|c|c|c|c|c|c|c|c|}
\hline & \multirow{2}{*}{$\begin{array}{l}\text { Mean (sd) or } \\
\text { frequency }\end{array}$} & \multicolumn{10}{|c|}{ Correlations } \\
\hline & & 1 & 2 & 3 & 4 & 5 & 6 & 7 & 8 & 9 & 10 \\
\hline 1. Age & $18.8(2.3)$ & - & & & & & & & & & \\
\hline $\begin{array}{l}\text { 2. Educational level } \\
\text { ( } \% \text { higher) }\end{array}$ & $62 \%$ & .06 & - & & & & & & & & \\
\hline $\begin{array}{l}\text { 3. Urbanization } \$ \\
\text { (\% urban areas) }\end{array}$ & $48 \%$ & $.15^{*}$ & .10 & - & & & & & & & \\
\hline $\begin{array}{l}\text { 4. Self-labeling } \$ \\
\text { (\% homosexual) }\end{array}$ & $85 \%$ & .01 & .01 & -.04 & - & & & & & & \\
\hline 5. Self-acceptance & $88.3(15.3)$ & -.07 & .07 & -.04 & $-.14^{*}$ & - & & & & & \\
\hline 6. IHN & $1.5(.60)$ & .08 & -.03 & .04 & -.01 & $-.39^{* *}$ & - & & & & \\
\hline 7. Disclosure & $78.5(20.7)$ & -.02 & -.05 & -.10 & $-.16^{*}$ & $.33^{* *}-$ & $-.37^{* *}$ & - & & & \\
\hline $\begin{array}{l}\text { 8. Discrimination } \$ \\
\text { (\% Yes) }\end{array}$ & $29 \%$ & $-.25^{* *}$ & ${ }^{*}-.32^{* *}$ & -.07 & -.04 & .04 & -.06 & $.17^{*}$ & - & & \\
\hline 9. Connectedness & $49.5(25.8)$ & -.02 & $-.16^{*}$ & .09 & -.09 & $.21^{* *}-$ & $-.34^{* *}$ & $.29^{* *}$ & $.15^{*}$ & - & \\
\hline 10. Depression & $1.1(.94)$ & $-.20^{* *}$ & ${ }^{*}-.17^{*}$ & -.03 & .04 & $-.14^{*}$ & $.14^{*}$ & -.08 & $.32^{* *}$ & .02 & - \\
\hline
\end{tabular}


Second, as would be expected, the minority-stress factors relating to coming-out were all correlated (all rs between .21 and .39; all ps<.01), except for self-labeling. Respondents who self-labeled as bisexual reported lower levels of self-acceptance $(\mathrm{r}=-.14, \mathrm{p}<.05)$ and disclosure $(\mathrm{r}=-.16, \mathrm{p}<.05)$; no other coming-out variables were related to selflabeling. Finally, discrimination was positively correlated with disclosure $(r=.17 ; \mathrm{p}<.05)$ and connectedness to the gay scene $(r=.15$; $\mathrm{p}<.05)$ and negatively correlated with age $(\mathrm{r}=-.25, \mathrm{p}<.01)$ and educational level $(\mathrm{r}=-.32, \mathrm{p}<.01)$.

Next, of the five of regression-analyses testing the moderating effect of connectedness, only the interaction between internalized homonegativity and connectedness was significant $(\beta=-.17, \mathrm{p}<.05)$. A subsequent stepwise regression-analysis with the interaction between internalized homonegativity and connectedness and the five minoritystress predictors entered in step 1 , and age and educational background entered in step 2 , showed a significant interaction term $(\beta=-.15, \mathrm{p}<.05)$, and a significant main effect of discrimination $(\beta=.35, p<.001)$. This model containing the five predictors and the interaction term explained $18 \%$ of the variance in depression (see Table 6.2). Adding age and educational background in the second step did not result in a significant increase in the amount of explained variance in depression $\left(\mathrm{R}^{2}\right.$ change $=.02$, F-change $\left.=2.02, \mathrm{p}>.05\right)$. 
Table 6.2 Prediction of depression from minority-stress factors and demographics (all reported $\beta$ 's are significant at $p<.05$ ).

\begin{tabular}{lccc}
\hline Predictors & $\begin{array}{c}\text { Zero-order } \\
\text { correlation }\end{array}$ & $\boldsymbol{\beta}$ Step $\mathbf{1}^{\mathbf{s}}$ & $\boldsymbol{\beta}$ Step $\mathbf{2}^{\text {s }}$ \\
\hline Discrimination & .32 & $.35^{* * *}$ & $.30^{* * *}$ \\
Self-label & .03 & $\mathrm{~ns}$ & $\mathrm{~ns}$ \\
Self-acceptance & -.15 & $\mathrm{~ns}$ & $\mathrm{~ns}$ \\
IHN & .15 & $\mathrm{~ns}$ & $\mathrm{~ns}$ \\
Disclosure & -.09 & $\mathrm{~ns}$ & $\mathrm{~ns}$ \\
Connectedness & .00 & $\mathrm{~ns}$ & $\mathrm{~ns}$ \\
Connectedness x IHN & -.17 & $-.15^{*}$ & $-.15^{*}$ \\
Age & -.20 & & $\mathrm{~ns}$ \\
Educational background & -.17 & & $\mathrm{~ns}$ \\
& & & \\
$\mathrm{R}^{2}$ & & .18 & .20 \\
Df (Change) & & 7 & 2 \\
Df(error) & & 171 & 169 \\
F & & 5.248 & 2.018 \\
\hline
\end{tabular}

\$Standardized beta

" $\mathrm{p}<.05,{ }^{* *} \mathrm{P}<.01,{ }^{* * *} \mathrm{P}<.001$

Finally, probing the interaction while correcting for age, educational level and the other four minority-stress factors showed that the simple slope is .417 at $-1 \mathrm{sd}(\mathrm{p}<.05),-.159$ at the mean of connectedness ( $p>.05)$, and -.099 at $+1 \mathrm{sd}(\mathrm{p}>.05)$. Thus, the effect of IHN on depression is only significant for relatively low values of connectedness, indicating that only YGBM who hold negative attitudes towards homosexuality and who report low levels of connectedness with the gay scene report increased feelings of depression.

\section{Discussion}

The present study aimed to test the relationship between minoritystress and emotional distress as suggested by the minority-stress framework [2] in a sample of YGBM who are in the midst of their coming-out. As suggested by this framework, both internal (i.e. self- 
labeling, self-acceptance, internalized homonegativity and level of disclosure) and external minority-stress factors (i.e. experienced discrimination) were hypothesized to be predictors of mental health. Furthermore, it was hypothesized that connectedness with the gay scene would moderate any relationship between minority-stress factors and mental health. In this study, mental heath was operationalized as feelings of depression as measured by the BSI $[148,149]$. In line with the minority-stress framework [2], both external (i.e. experienced discrimination) and internal (i.e. internalized homonegativity) factors were significantly related to feelings of depression. Moreover, connectedness with the gay scene only moderated the relationship between internalized homonegativity and depression, in that the effect of internalized homonegativity on depression was only significant for YGBM who report low levels of connectedness with the gay scene.

Of the minority-stress factors examined in the present research, experienced discrimination was the single best predictor of increased feelings of depression. There is a growing body of literature that suggests that the experience of discrimination can have a negative impact on mental health in gay and bisexual men [30, 138, 144, 154, 155] and our findings are consistent with this literature. The positive correlation between disclosure and experienced discrimination and between connectedness with the gay scene and discrimination suggests that YGBM who are more "out" about their sexual orientation and who feel more connected with the gay scene experience more discrimination. In addition to experienced discrimination, internalized homonegativity (i.e. holding a negative attitude towards homosexuality) was significantly correlated with reporting more depressive symptoms, but was not significant in the multivariate analysis. The hypothesized ameliorative effect of connectedness with the gay scene emerged only for internalized homonegativity. More specifically, probing the interaction revealed that the effect of internalized homonegativity on depression was only significant for respondents who reported low levels of connectedness with the gay scene. 
In contrast to some studies $[32,156]$, and in line with other studies [133], coming-out related minority-stress factors were not related to reporting more depressive symptoms. There are several possible explanations for this somewhat unexpected finding. First of all, we used different scales and measures to assess the various comingout variables. For example, our measure of disclosure did not consist of a proportion of person a respondent disclosed their identity to (i.e. a more objective level of disclosure), but rather a subjective measure of disclosure (i.e. to what extent do you consider yourself to be "out"). Moreover, as suggested recently, perhaps it is not disclosure itself, but rather acceptance or rejection of sexual orientation by others that lead to feelings of depression [156]. Second, our sample consisted of YGBM who were recruited both online at various gay-related Internet sites and offline at various gay-friendly bars and parties. This may have resulted in a sample of YGBM who are already quite comfortable with their sexual orientation. Third, recently a study suggested that the reason why some studies fail to find associations between minoritystress factors and emotional distress may be because YGBM differ in the amount of social constraints they experience [157]. Social constraints are perceived barriers and difficulties that prevent, in this case, YGBM from talking with others about one's problems and difficulties related to being a sexual minority. It has been shown that lesbians who are high in stigma consciousness (i.e. the expectation of being stigmatized or discriminated because of sexual orientation) and who also perceived high social constraints in talking with other lesbians were more likely to report emotional distress [157]. Finally, most research on well-being of YGBM during their coming-out is conducted in the United States [28, 133, 156, 158, 159], a country known to be less tolerant towards homosexuality. It is possible that a gay-unfriendly environment may result in more problematic comingout experiences for YGBM. For example, YGBM may have more fear of rejection because of their sexual orientation, which may subsequently withhold YGBM to disclose their sexual identity to others. In contrast to the US, the Netherlands is a relatively gay 
tolerant country [77] and perhaps these fears of rejection are less prevalent.

Finally, the mean score on the BSI-depression scale on the current study is considerably higher compared to the mean score of the Dutch general population (i.e. a mean score of 0.40 on the BSI-depression scale) and lies closer to the mean score of Dutch psychiatric patients (i.e. 1.58; [149]). This suggests that YGBM in the current study are vulnerable to depressive symptoms, a finding which is consistent with other research [32, 155, 158, 160, 161]. However, this finding should be interpreted with extreme caution. First of all, no control group of heterosexual male adolescents between 16 and 25 years of age was included in the present research. Furthermore, no BSI norm scores are available for Dutch adolescents younger than 20 years of age in the Netherlands. Therefore, we cannot conclude that our sample of YGBM is at increased risk for depressive symptoms compared to their Dutch heterosexual peers.

Some limitations of our research should be discussed. First of all, as already noted above, no control group was recruited which made it impossible to assess differences in reported depressive symptoms between YGBM and their heterosexual counterparts. Moreover, because the Outcomes questionnaire was already quite lengthy not the complete BSI was used but only the BSI-depression scale. As a result, we could not calculate an overall score of emotional distress. Second, respondents were included based on a measure of sexual orientation (on a 5 -point scale), and respondents who indicated to be attracted exclusively to women or not exclusively but to a great extent, were excluded from the present research. This may have led to an underrepresentation of respondents who are attracted to women but occasionally have sex with men. Third, the present research only included one measure of well-being. As a recent meta-analysis showed, lesbian, gay, and bisexual persons are also at increased risk of other health problems such as anxiety disorders, suicide ideation, alcohol and other substance dependence [160]. Fourth, because our sample consisted of YGBM who were in the midst of their coming-out at study entry, the present findings cannot be generalized to all YGBM. Finally, 
our data are cross-sectional and therefore we can not establish causality.

In conclusion, the present study showed that YGBM who experienced discrimination because of their sexual orientation report more depressive symptoms compared to YGBM who did not experience discrimination. Furthermore, YGBM who hold negative attitudes towards homosexuality and who do not feel connected with the gay scene report more depressive symptoms. From a practical point of view, this research underscores the need to design interventions to prevent anti-gay harassment and to stimulate the acceptance of sexual minorities. 



\section{Chapter 7 General discussion}

In the present thesis, the results of Outcomes, an online cohort of young gay and bisexual men, were reported. One of the main aims of Outcomes was to explore sexual risk behaviors of YGBM who were at study entry - in the midst of their coming-out. In addition, correlates of mental health (e.g. depressive symptoms) of these YGBM were examined. In this general discussion, the main results of the studies presented in this thesis will be briefly summarized and discussed. Next, a summary will be provided of the different risk factors examined in the studies presented in this thesis. Furthermore, suggestions for intervention development will be provided. Finally, the limitations of studies will be reported.

\section{Sexual debut}

The main reason to start with Outcomes was the recent increase in sexual risk behavior observed among YGBM, both in the Netherlands $[5,6]$, and elsewhere [46, 51]. Although the prevalence of HIVinfection among YGBM is relatively low, the fact that some YGBM already contracted HIV is reason for concern. Moreover, it is possible that an unsafe start of their sexual career may inform behavior later on in their sexual careers. It is therefore important to know how many YGBM start their sexual career unsafe and to understand the reasons why YGBM engage in UAI with their first male sexual partner(s). One first remark: it should be noted that the first episode of same-sex intercourse does not necessarily involve penetrative sex.

In chapter 2, three research questions were addressed: 1) where do YGBM in the Netherlands meet their first same-sex partner; 2) how 
many YGBM use a condom during their first episode of anal intercourse; and 3) does meeting the first same-sex partner online facilitates sexual risk behavior? The results showed that many, but not all, YGBM in the Netherlands are active online; only two respondents never visited any gay-related websites. In addition, most YGBM were very active online, with about half visiting gay-sites on a daily basis. However, despite the fact that many YGBM are active online, only about half of respondents met their first same-sex partner online. About half of the YGBM (i.e. 45\%) reported UAI with their first samesex partner and, in contrast to other studies [36, 44], meeting the first same-sex partner online was not associated with more sexual risk taking behavior.

The Internet seems to play an important role in many YGBM's life nowadays, not only with respect to seeking same-sex partners [35], but also with respect to other aspects of homosexuality such as coming-out $[34,162]$. Notwithstanding the popularity of the Internet, it is not to be seen as the only channel through which YGBM meet their sex-partners. In the Netherlands there is quite an active scene for YGBM. For example, during recruitment we discovered that there are quite some gay parties specifically targeting YGBM (between 16 and 25 years of age) and about half of respondents were recruited at such venues. Also, YGBM who are active at gay-related Internet sites (mostly at www.expreszo.nl, which is a site specifically for young LGBs) regularly organize meetings at such parties or organize trips to festivals or theme parks. This raises an important question with respect to online dating: should all partners met online (especially online first same-sex partners) be seen as online partners in the sense of "actively sought" online partners? Most research on online sexual risk behavior assume or assess whether respondents were "actively" seeking sex partners online [38, 44, 163]. One could argue however that not all YGBM who met their first same-sex partner online (or offline) had an explicit intention to have sex. As explained above, it is possible that initial contact with their first same-sex partner occurred online but that they nevertheless met this particular person under different circumstances and with a different intent offline (e.g. at YGBM 
meeting organized by a certain gay-web community). More research is necessary to understand the specific circumstances under which YGBM meet their first anal same-sex partner.

How can we explain the high rate of UAI during the first episode of anal intercourse reported by respondents? Research has shown that large age discrepancies between partners may result in power differences, with older partner exerting pressure to engage in unprotected sex [39, 126, 164]. Partial support for this affirmation comes from chapter 3 in which it was shown that on average, the first same-sex partner was two years older. However, this age difference refers to the first same-sex partner and not necessarily to the first anal same-sex partner. Moreover, a proper testing of this assertion would entail assessing whether social pressure to engage in UAI was applied by the first anal same-sex partner. A second explanation could be that YGBM may have a biased HIV risk appraisal. For example, they might underestimate the odds that their first same-sex partner could be HIVinfected. Moreover, they might reason that because they never engaged in anal intercourse before, they themselves cannot be infected with the HIV-virus. Although in itself this is true, the possibility of an age discrepancy between partners and therefore perhaps a sexual experience discrepancy as well may still facilitate HIV-transmission and other STIs between age cohorts. More (qualitative) research is necessary to determine why YGBM engage in UAI during sexual debut and what influenced the decision to engage in UAI.

In chapter 2 and 3, information was presented about condom use during the first episode of anal intercourse. These findings raised a number of research questions which need to be addressed in future research. First of all, future research needs to establish whether the first episode of anal intercourse occurred before or after the coming-out and whether this coming-out sequence is associated with the decision to engage in UAI with the first same-sex partner (see for example [10, 13]). Second, it should be examined whether the first episode of anal intercourse occurs during sexual debut. If sexual debut does not involve anal intercourse than future research should also assess the number of sexual episodes prior the first episode of anal intercourse 
(i.e. as a measure of sexual experience) and whether sexual experience influences the decision to engage in UAI with the first anal same-sex partner. In addition, power differences between partners should be assessed and it should be examined whether power differences may explain sexual risk behavior with the first anal same-sex partner. In terms of differences in sexual risk behaviors between online versus offline partners, future research should assess whether YGBM are actively seeking sex online when they meet their first same-sex partner online. This difference in sex seeking behavior may be important in understanding sexual risk behavior with first anal same-sex partners. Finally, in order to guide intervention development, the social-cognitive determinants of condom use during sexual debut should be assessed. It is possible that the determinant configuration of the first anal same-sex partner differs from the determinant configuration of subsequent episode of anal intercourse as reported in chapter 5.

In conclusion, although we do not yet fully understand the circumstances in which the first episode of anal intercourse occurs, the findings from chapter 2 and 3 do indicate that many YGBM start their sexual career unsafe. Meeting the first partner online does not seem to facilitate UAI with the first same-sex partner. More research is necessary to determine the circumstances surrounding the first episode of anal intercourse. Finally, preventive interventions are necessary to inform and motivate YGBM to use condoms at their sexual debut and these interventions must target YGBM preferably before their sexual debut.

\section{Cross-sectional and longitudinal risk behaviors}

In chapter 3 results were reported from the baseline measurement of sexual risk behavior per partner type. In addition, correlates of UAI per partner type were examined. In chapter 4 , longitudinal patterns of sexual risk behavior were presented and correlates of longitudinal risk behavior were examined. In this section an overview of the findings on cross-sectional and longitudinal risk behaviors will be presented.

The study presented in chapter 3 showed that a significant proportion of YGBM engage in sexual risk behaviors with steady, 
regular and casual partners. Moreover, in three subsequent waves of Outcomes these rates of UAI per partner type remained relatively similar (data not presented in the present thesis, but see [34, 162]). The findings from chapter 3 correspond reasonably well with other MSM Monitoring studies conducted in the Netherlands [5, 6] and elsewhere $[4,9,165]$. For example, about $35 \%$ of MSM who have had sex with casual partners reported at least one episode of UAI with a casual partner in the six months preceding assessment $[5,6,9,66]$. Thus, in terms of condom use behavior with casual partners, YGBM at the beginning of their sexual trajectory already seem to resemble older MSM in the Netherlands. However, as already noted in chapter 3, there was also a remarkable difference between YGBM and their older counterparts. In Outcomes, fewer YGBM had anal sex with casual partners, but those who did have anal sex reported more UAI. One explanation for the relative high rates of UAI with casual partners could be that YGBM are not yet fully aware of the risks on contracting HIV. For example, since relatively few YGBM in the Netherlands are infected with HIV, the disease may be less visible in this age cohort and because of this lack of visibility YGBM may be inclined to believe that HIV poses no health risk for them. In addition and related to this, it is possible that YGBM regard HIV-infection as a disease which is more prevalent among older cohorts of MSM and more specifically, among older MSM who they consider to be more promiscuous. In terms of prevention, although risk appraisal in itself is not sufficient for behavior change, YGBM must be made aware of the fact that their line of reasoning may be flawed. Moreover, YGBM should be made aware that at some point they themselves become part of this older age cohort of MSM, in which HIV and other STIs are more prevalent.

The rate of UAI for steady partners was somewhat lower compared to other studies but these rates tended to increase with relationship duration (see chapter 3). Anecdotal evidence (based on feedback during recruitment and on feedback from interviewers of the qualitative part of Outcomes) suggests that YGBM seem to be rather quick in labeling someone as their steady partner even though they have known each other for a relatively short period of time. For 
example, one respondent indicated that he had been chatting online with a particular person for a few weeks and even though they had not met in person yet, he already considered this person as his steady partner. This may in part also explain the high relationship turnover rates observed in other studies [43]. For example, in Outcomes the average relationship duration with the first steady partner was 4 months, with about half of YGBM who ever had a relationship reporting relationship duration between 1 and 3 months. Despite this high relationship turnover rate, about half of YGBM do not use condoms with their steady partner. It is possible that YGBM hold naive and romantic ideas about relationships and therefore believe that using condoms with a steady partner is not necessary. Given the high relationship turnover rate and as a result the possibility to be involved in many successive relationships (i.e. serial monogamy), it is recommended that prevention messages must communicate that condoms should always be used, even in steady relationships.

Based on observations in recent Dutch MSM Monitoring studies $[5,6]$, the concept regular partner was introduced as a new partner category. The results presented in chapter 3 show that regular partners seems to be a relevant partner category because more YGBM reported sex with regular compared to casual partners. However, little is known about the dynamics of sex with regular partners. For example, it is possible that some regular partners are so-called "fuck buddies" (i.e. a steady sex partner as opposed to a romantic steady partner). It is also possible that a regular is a "special" casual partner with whom one has had sex with before but who is not considered to be a fuck buddy. For example, the first episode of sex with a particular casual partner occurred some time ago and after meeting this particular partner again (e.g. accidently in a bar or through friends) one has sex with him again. Another possibility is that a regular partner is someone who has the potential to become a steady romantic partner but has not yet been labeled as such (i.e. in the process of becoming involved in a steady relationship). In the quantitative part of Outcomes the specific characteristics of regular partners was not assessed. Future research should investigate the concept regular partners in more detail (for 
example through qualitative research). Moreover, reasons why YGBM engage in sexual risk behavior with regular partners must be determined.

In order to gain more insight into longitudinal sexual risk behaviors of YGBM, in chapter 4, YGBM were classified based on their sexual behaviors during each of four waves of Outcomes. Based on previous research on longitudinal risk behavior of MSM in the Netherlands [97], respondents were classified as consistent no risk (i.e. no sex, no anal intercourse or no UAI at all four data waves), consistent risk (i.e. UAI at all four waves of Outcomes) or variable risk (i.e. any combination of no risk and UAI over four data waves). As can be seen in chapter 4 , a substantial proportion engaged in variable risk behavior (i.e. $45 \%$ ) and a smaller proportion of YGBM engaged in unsafe sex in each of four waves of Outcomes (i.e. 14\%). It should be noted however, that these longitudinal risk behaviors were not stratified per partner type. This means that the longitudinal sexual risk behaviors reported in chapter 4 are based on a composite measure consisting of sexual behavior with steady, regular and casual partners. The main reason for not stratifying based on partner type was to increase statistical power. The result of this decision however was a loss of information about longitudinal patterns of UAI per partner type. For example, research has consistently shown that the rate of UAI with steady partners differs from casual partners in that higher rates of UAI are observed with steady partners [5, 6, 59]. This limitation should be kept in mind when interpreting the results of chapter 4 .

Chapter 4 showed that about $14 \%$ of YGBM in Outcomes consistently engaged in UAI in each of four waves of Outcomes. Although some risk factors were examined in relation to consistently engaging in UAI (see next section), it is unknown what characterizes these YGBM. For example, it is possible that the consistent risk group consists of YGBM who intentionally engage in sexual risk behavior. In contrast, the variable risk group may consist of YGBM who perhaps accidentally engaging in sexual risk behavior. If YGBM in the consistent risk group intentionally engage in sexual risk behavior than one might expect that these YGBM have a significantly lower intention 
to always use condoms compared to YGBM in the variable risk group. As reported in chapter 4, no such difference in intention was observed. Given the paucity in longitudinal research on sexual risk behaviors and associated risk factors of YGBM, more longitudinal research is necessary to determine reasons for consistently or variably engaging in UAI. Moreover, future studies should examine longitudinal risk behaviors for different partner types and should test whether the structure of risk factors is similar for each partner category.

Summarizing, after a risky sexual debut, many YGBM continue to practice unsafe sex with steady, regular and casual partners. Moreover, across four waves of Outcomes, about $14 \%$ consistently engaged in sexual risk behaviors, and about $45 \%$ had at least one episode of UAI in this time frame. These behavioral data indicate that preventive interventions are necessary to inform and motivate YGBM to continue practicing safe sex.

\section{Determinants of sexual risk behavior}

The previous sections showed that many YGBM engage in sexual risk behaviors, which warrants for the development and implementation of interventions promoting safe sex. In order to guide the development of these interventions, the determinants of condom use have to be known $[22,86]$. In chapter 3 and 4 , several the behavioral and cognitive determinants of sexual risk behavior were examined and reported. Moreover, in chapter 5, the social-cognitive determinants of condom use with casual partners were examined and reported. This section provides a brief overview of the risk factors examined in the present thesis. For the sake of readability, this section is sub-divided into behavioral and cognitive risk factors.

\section{Behavioral determinants}

First of all, in contrast to several other studies, in the present thesis demographic factors (i.e. cultural background, educational status, urbanization, and religious beliefs) were not significantly associated with sexual risk behavior (see chapters 3 and 4). Several studies showed that more risk taking behavior is reported by Blacks and 
Hispanics compared to non-white YGBM (predominantly in the US; [4, $47,166])$, by rural compared to urban YGBM, by lower educated compared to higher educated YGBM [167], and by non-religious compared to religious YGBM [168]. Most models on determinants of sexual risk behavior assume that demographic factors are more distal factors and that their influence on behavior goes via more proximal factors such as knowledge or attitudes (e.g. [25]). For example, some studies have found that sexual risk taking behavior is more prevalent in lower compared to higher educated MSM. However, this relationship could be explained by the fact that lower educated MSM also have less knowledge regarding HIV-risk [167]. It should be noted that the absence of significant associations between demographic factors and sexual risk behavior in the present thesis should be taken to mean that these factors are not important for intervention development. Even though demographic factors are usually not amenable to change, these factors can still provide valuable information for the selection of different target groups [22, 86].

Second, one of the most important risk factors that has consistently be associated with sexual risk behavior is partner status (e.g. $[5,6,59,169,170])$. As can be seen in chapter 3 , more UAI was reported with steady partners (i.e. $43 \%$ ) compared to regular or casual partners (i.e. both $33 \%$ ). Moreover, the odds on UAI with a steady partner increased with relationship duration, also a common finding in MSM research [43]. Although the proportion of YGBM engaging in UAI is considerably higher for steady partners compared to casual partners, this does not necessarily mean that YGBM in steady relationships are at increased risk of acquiring HIV or other STIs. It is possible that YGBM in steady relationships practice a harm reduction strategy referred to as "negotiated safety", a strategy where sexual partners in steady relationships practice UAI provided that both partners are HIV-negative and that both partners make clear arrangements about safe sex outside the relationship. Thus, partners must either agree not have sex with other MSM outside the relationship or must agree only engaging in protected intercourse with MSM outside the relationship. Also, the strategy prescribes that both 
partners take two separate HIV-tests; one at the beginning of the agreements and a second test after three months (i.e. consistent with the incubation period for the HIV-virus). Finally, partners must disclose to each other whenever UAI has occurred outside the primary relationship and in case this occurred are required to take two HIVtests again. It is questionable whether YGBM in Outcomes already practice negotiated safety in steady relationships. First, a Dutch study showed that only $12 \%$ of YGBM in steady relationships practiced negotiated safety as a harm reduction strategy [42]. Second, the observed HIV-testing rates are rather low with 17\% of YGBM ever being tested for HIV prior the first wave of Outcomes (see chapter 3). Third, the YGBM who were tested for HIV report on average reported just one HIV-test. Finally, given the assumed high rate of relationship turnover, it is difficult for YGBM to adhere to the requirements of negotiated safety (i.e. taking two HIV-tests before engaging in UAI with their steady partner). Therefore, we do not recommend promoting negotiated safety as a primary HIV harm reduction strategy for YGBM. Instead, primarily because of high rates of relationship turnover (see also discussion in previous section), prevention message must urge YGBM to always use condoms even when involved in a steady relationship.

Third, consistent with the literature [20,38, 46, 68], we observed a significant association between reporting multiple lifetime same-sex partners and UAI with casual partners (chapter 3 ) and with longitudinal risk behaviors (chapter 4). Having multiple sexpartners automatically increases the opportunity to engage in UAI. However, as already noted in chapter 4, any UAI is a relatively crude measure of actual risk in that some YGBM who report multiple sex partners may have engaged in only one episode of UAI compared to other YGBM who may have engaged in multiple episodes of UAI [104]. Nevertheless, prevention may be directed towards creating awareness that having multiple sexpartners also increases the odds on contracting HIV and other STIs, especially when engaging in UAI with most or all of these partners. 
Finally, prior research has shown that HIV-testing is related to UAI $[62,88]$. In chapter 4 we also found a significant association between consistently engaging in UAI and ever being tested for HIV. However, as already noted in chapter 4, the relationship between HIVtesting and sexual risk behavior is not yet clear. It is possible that YGBM who consistently engage in UAI have a good understanding of HIV-risk related to UAI and therefore decide to get (frequently) tested on HIV. At the same time, it is possible that YGBM consider a negative test result as an argument to continue practicing UAI (i.e. the test was negative thus the risk on HIV must be low, therefore I will continue to practice UAI). Future studies should examine the relationship between HIV-testing and sexual risk behavior. Also, future studies should assess the main reasons for YGBM to get tested for HIV. Potential reasons could be having engaged in UAI, just wanting to know their status, or for medical reasons. The finding that more HIV-testing is reported by YGBM who consistently engage in sexual risk behavior, is in a sense also good news. Not only is it promising that they decided to get tested, but post-test counseling also provides health care workers with the opportunity to inform YGBM about the risk of UAI and to persuade YGBM to always use condoms.

In sum, in the studies described in this thesis little evidence was found for the predictive power of demographics as risk factors. Furthermore, more sexual risk taking behavior was reported by YGBM who are involved in a steady relationship, by YGBM who report multiple lifetime same-sex partners and by YGBM who ever have been tested for HIV.

\section{Cognitive determinants}

First of all, in contrast to some studies [91, 93, 108] and in line with other studies [92, 94, 107, 171], a significant positive association between HIV-optimism and longitudinal sexual risk behavior was observed. Although YGBM seem to be quite realistic about the consequences of HAART (for example, most YGBM disagreed with the statement that with the availability of HAART, having protected intercourse is no longer required), YGBM who are more inclined to agree with this and similar statements about HIV-treatment are also 
more likely to engage sexual risk behavior (see chapter 4). More specifically, of the three subscales used to measure treatment optimism (i.e. treatment optimism, safe sex necessity, safe sex fatigue), only the safe sex fatigue scale was significantly associated with sexual risk behavior. Safe sex fatigue refers to feelings of indifference or apathy towards always engaging in protected anal intercourse. It is surprising that some of the YGBM in Outcomes already express feelings of safe sex fatigue. On the one hand, respondents in Outcomes are young and assumed to be at the relative beginning of their sexual trajectory. Usually, it is assumed that feelings of safe sex apathy are more prevalent in older, more experienced MSM. On the other hand, it is also possible that YGBM who are more inclined to report feelings of safe sex fatigue are sexually more experienced (e.g. report more lifetime same-sex partners) compared to the other YGBM in Outcomes. Nevertheless, the finding that YGBM who report unsafe sexual behavior also express feelings of safe sex apathy is worrying. Given that HIV-treatments have improved and most likely continue to improve (thereby reducing infectiousness of HIV), this may provide YGBM with strong arguments to continue practicing unsafe sex. However, unsafe sex also constitutes risk on contracting other STIs. Therefore, HIV-prevention messages must not only focus on the risks on contracting HIV but also on contracting other STIs, for example by stressing that becoming infected with HIV is not the only health consequence of unsafe sexual behavior.

Second, social-cognitive determinants (e.g. intention, attitudes, social norms, perceived behavioral control, personal norms, and behavioral willingness) have been shown to be related to condom use. In chapter 5 a study was reported that sought to investigate to what extent these factors influence condom use with casual partners. The theory of planned behavior, TPB [25], served as theoretical background (see chapter 5 for more details on the utilization of this theory in the present research). The merits of the TPB in predicting condom use intention and actual condom use has been demonstrated by a large body of research (for review see [95]). The TPB (and other socialcognitive models) assume that having a positive intention to perform 
the desired behavior is a first prerequisite for performing this behavior $[25,127]$. In line with this, it was shown in chapter 5 that behavioral intention (i.e. the intention to always use condoms when having anal intercourse with a casual partner) was positively associated with actual condom use with casual partners and explained $28 \%$ of the variance in behavior. Moreover, in the longitudinal study reported in chapter 4, we also found that behavioral intention was negatively associated with consistently or variably engaging in UAI in four waves of Outcomes. Thus, behavioral intention seems to play an important role in the decision to engage in protected anal intercourse with casual partners.

Third, according to the TPB, intentions are predicted by attitudes, subjective norms, and perceived behavioral control [25]. In line with a large number of studies, it was found that expressing positive attitudes towards condom use was related to behavioral intention. More specifically, both positive beliefs (i.e. condoms are hygienic and creates feeling of being safe), and negative beliefs (i.e. condoms are an irritating disturbance, creates distrusts, and are less intimate) discriminated between YGBM with a high and YGBM with lower behavioral intention. Social influences may come from two sources: injunctive social norms (i.e. what you think your social referents think you should do) and descriptive social norms (i.e. what you think your social referents do). In chapter 5 , no evidence was found that injunctive social norms are related to behavioral intention. In fact, a substantial proportion of YGBM (i.e. about $40 \%$ ) indicated not knowing what their social referents think they should do. Interestingly, even though a significant proportion indicated not knowing what their best gay friends think they should do, only $13 \%$ (percentage not reported in this thesis) indicated not knowing whether their best gay friend uses condoms when having anal intercourse with casual partners. However, the latter may also reflect an inference based on the behavioral norm and on assessing whether their social referent will conform to this norm. It should also be noted that excluding the YGBM who indicated not knowing what their social referent think they should do from the analysis, did yield a significant association between injunctive social norms and behavioral intention. The fact that 
a large proportion of YGBM did not know what their social referents think they should do, could indicate that YGBM do not yet talk about condom use with their social referents. In fact, a focus group interview study conducted among YGBM in the Netherlands showed that YGBM rarely discuss safe sex with their friends [172]. However, in contrast to our findings, the YGBM in the focus group study indicated also not being aware of safe sex behaviors of their social referents. This may provide further evidence that YGBM do not talk about condom use and that the descriptive norm in part reflects an inference based on assumptions rather than on actual knowledge about condom use of social referents. Future studies should investigate the role social referents could play in promoting condom use. In line with a large number of studies [95], we found that perceived behavioral control was associated with a positive intention to use condoms with casual partners, but not with actual condom use. More specifically, YGBM with high behavioral intention were also more likely to express feelings of control about condom use in specific circumstances (such as when in love, when partner is attractive, and when sexually aroused), about communicating condom use with same-sex partner such as telling partner to use condoms if partner does not want to for insertive or receptive anal sex, and about applying condoms to themselves and their partner. Given the fact that in general perceived behavioral control is directly associated with condom use behavior, it is possible that YGBM in Outcomes did not have relevant experience with the situations described in the control beliefs. For example, it is possible that YGBM have little or no experience with partners who explicitly state they want to have unprotected sex or with applying condoms to themselves or their partners. Also, the relative small number of YGBM who reported anal intercourse with a casual partner (i.e. 31 respondents), may have resulted in a lack of statistical power.

Fourth, YGBM who hold strong personal norms regarding condom use with casual partners were also more likely to express a positive intention to always use condoms. Some researchers have argued that because personal norms are very strongly related to intentions they act as a sort of intention. Other researchers have 
suggested that if behavioral intentions are based upon personal norms, these intentions will better predict behavior [118]. The aim of the study presented in chapter 5 was not to test these hypotheses, but to examine whether personal norms add in the prediction of behavioral intention to always use condoms. More specifically, YGBM who belief that it is normal to use condoms, that they would feel guilty if they did not use condoms, who feel it is against their principles to not use condoms, and who feel morally obliged to use condoms have a higher intention to use condoms.

Finally, in contrast to theory [75], behavioral willingness (i.e. the willingness to engage in sexual risk behavior given certain settings) did not predict actual condom use. As stated above, this could be the result of a lack of experience with the behavior or due to a lack of statistical power. However, behavioral willingness did significantly differentiate between YGBM with a high compared to a lower intention. YGBM with a lower intention are more willing to engage in sexual risk behavior when the partner says or when they think he is HIV-negative, when no condoms are available, or when they expect this partner to become a regular or steady partner.

In conclusion, next to the influence of behavioral factors (i.e. relationship status, number of lifetime same-sex partners, HIV-testing) on condom use, several cognitive factors (i.e. intention, attitudes, perceived behavioral control, descriptive and personal norms, and behavioral willingness) have also been found to influence condom use in YGBM. The resulting determinant configuration based on the risk factors for UAI described above is presented in Table 7.1. Before turning to a discussion of the practical implications, the next section discussed the results of chapter 6 in which a study was reported on predictors of mental health outcomes in YGBM.

\section{Mental health}

Although the primary health risk in YGBM is usually considered to be HIV and other STIs, mental health concerns are also prevalent in this population [2]. The higher prevalence of mental health concerns observed among YGBM is commonly explained in terms of minority- 
stress; a set of unique stressors specifically related to belonging to a sexual minority [2]. In chapter 6 a study was reported which investigated the relationship between minority-stress factors and mental health (operationalized as depressive symptoms). The results showed that YGBM who experienced discrimination and/or who do not feel connected with the gay scene and who have negative attitudes towards homosexuality are at increased risk of reporting depressive symptoms.

One question which was not addressed in the study presented in chapter 6 was whether mental health concerns (e.g. depressive symptoms) are associated with sexual risk behavior. There is some research evidence for this link between mental health concerns and sexual risk behavior in YGBM. For example, Gold and Skinner [173] found that a negative mood state (defined as depression, stress, and mental or physical tiredness) was associated with unsafe sex in a sample of YGBM. Another study, investigating the relationship between stress, coping, and unprotected sex, found no evidence for a direct link between stress and sexual risk behavior [174]. However, the authors did found a link between coping and sexual risk behavior in that MSM who reported UAI were more likely to use sex as a coping strategy for stress. A more recent study found an association between depression and reporting three or more same-sex partners in the 6 months prior assessment [137]. However, no significant association was found between depression and unprotected sex. Evidence against the link between mental health concerns and sexual risk behavior comes from a recent meta-analysis of 34 studies examining the link between negative affective states and unprotected sex. The authors found no significant association between depression or anxiety or anger and sexual risk behavior [175]. Although the primary aim of the study reported in chapter 6 was to investigate the relationship between minority-stress factors, it provided us with the opportunity to examine associations between minority-stress factors, depression and sexual risk behavior. No significant associations were observed between minoritystress factors and sexual risk behavior. Moreover, no significant associations were observed between depression and sexual risk 
behavior. However, it is possible that depression may predispose YGBM to be involved in more risky situations whereas actual sexual risk behavior may be influenced by more proximal factors such as intentions to engage in sexual risk behavior [137]. However, until evidence is presented for an association between depression and sexual risk behavior, depression will not be considered a relevant risk factor for unsafe sexual behavior.

\section{Limitations}

The studies reported in the present thesis have several limitations which already have been discussed in the various chapters. This section briefly summarizes these limitations. First of all, the Outcomes study consisted of YGBM who were - at study entry - in the midst of their coming-out. In addition, because the Netherlands is known to be a fairly liberal country with respect to homosexuality, some YGBM may have their coming-out at an age younger than 16 . Therefore, the findings presented in this thesis cannot be generalized to all YGBM. Second, despite the fact that respondents were in the midst of their coming-out, the studies presented here did not report on potential associations between specific coming-out factors (e.g. self-acceptance, disclosure, internalized homonegativity) and sexual risk behavior. Third, recruitment took place at Internet sites and venues that cater to YGBM and this may have resulted in an overrepresentation of YGBM who already comfortable with their sexual identity. In addition, not all factors (e.g. illicit drug use prior or during sex, personality factors, family or partner violence, childhood sexual abuse) which may be associated with sexual risk behavior have been examined in the studies reported here. Fourth, the Outcomes study sample consisted of a limited sample size which may have resulted in a lack of statistical power. Finally, the studies reported in chapter 2, 3 and 6 are crosssectional which means that no conclusion can be drawn about causality. 


\section{Practical implications}

The development of effective interventions requires a systematic approach, in which both theory and research evidence must guide the development of these intervention [22, 86]. Intervention mapping provides a protocol for the development of theory- and evidence based interventions and consists of six steps. In short, the first step consists of conducting a needs assessment based on which program outcomes should be established. The second step is constructing matrices of change objectives which will serve as the building blocks of the intervention. The third step comprises seeking theory-informed and practical strategies to change the behavior of the target group. Step four consists of developing the actual program. Step five consists of planning the implementation of the intervention and the final step consists of the planning of the evaluation of the intervention. The research presented in the present thesis provided information for the first, second and the third step in this process.

The first step requires a needs assessment in which the health problem is determined and its related behavior and their determinants are assessed. In short, for YGBM, the health problem is infection with HIV or other STIs and the related behavior is unprotected anal intercourse. In the present thesis multiple behaviors have been identified (i.e. anal intercourse with the first same-sex partner, and anal intercourse with steady, regular and casual partners) and each behavior may require different intervention strategies. However, in the present thesis not all the determinants as outlined above (see also Table 7.1) were specifically examined for any of the four behaviors identified. For example, whereas the TPB determinants were analyzed for anal intercourse with casual partners, HIV-optimism was analyzed for any unprotected anal intercourse. Nevertheless, the determinant configuration outlined above provides valuable information for intervention development. 
Table 7.1 Determinant structure of sexual risk behavior.

\begin{tabular}{lc}
\hline Determinant & Relevance* $^{*}$ \\
\hline Traditional TPB determinants & +++ \\
Intention & ++ \\
Attitude & 0 \\
Subjective norm & ++ \\
Perceived behavioral control & \\
Additional determinants & + \\
Descriptive norm (best gay friend) & 0 \\
Descriptive norm (YGBM general) & +++ \\
Personal norm & 0 \\
Behavioral willingness & ++ \\
HIV-optimism (safe sex fatigue) & + \\
\hline
\end{tabular}

* The symbols $=$ and - denote positive and negative associations with sexual risk behavior. One symbol reflects a weak association, two symbols a moderate association, and three symbols a strong association. Zero's ( 0$)$ indicate no or trivial association.

Moreover, the behavioral determinants discussed above provide additional information for the selection of the at-risk population and for the content and the setting of the intervention. Nevertheless, program planners should be aware that different determinants configurations may exists for different sexual risk behaviors.

The second step in Intervention Mapping requires the selection of the determinants on which the intervention must be build. This selection must be based on two criteria: relevance and changeability. Whereas relevance refers to the strength of the association between the determinant and the behavior, changeability refers to the degree to which determinants are changeable through interventions. Table 7.1 provides an overview of the relevance of each of the determinants of sexual risk behavior studied in the present thesis. The determinant configuration in Table 7.1 should be complemented with information about the determinants' changeability [22]. Next, the resulting 
determinant configuration should be crossed with so-called performance objectives. A performance objective is defined as a subbehavior of the lowest possible level of the desired behavior. For example, for consistent condom use with casual partners during anal intercourse (i.e. the desired behavior) a performance objective could be to "tell a partner who wants unprotected sex to use a condom". Crosstabulating the performance objectives and the determinant configuration results in a matrix of change objectives (i.e. concrete intervention targets). An example of a change objective for the performance objective "telling a partner who wants unprotected sex to use a condom" and the determinant "perceived behavioral control" would be to "express confidence in one's ability to tell a partner to use condoms". These concrete change objectives must be targeted in an intervention. It is beyond the scope of the present thesis to state specific performance objectives, to cross-tabulate these performance objectives with the determinant configuration and to compose specific change objectives based on this matrix.

The next step (i.e. step three) would be to determine how these change objectives can be accomplished through an intervention. For a complete overview of theory-based behavioral change methods the reader is referred to chapter 7 in Bartholomew et al. [22]. One aspect of determining how these change objectives can be accomplished is to select practical strategies for delivering the intervention. Although the selection of practical strategies depends on the behavioral change methods associated with each change objective, the present research provides some information for the selection of practical strategies. The Internet seems to play an important role in the lives of many YGBM, and therefore program planners may be inclined to regard the Internet as a suitable setting to deliver the intervention. There are several benefits to delivering interventions online. First, because many YGBM at the beginning of their sexual trajectory are active online, the Internet may particularly suitable for reaching many YGBM. Second, the Internet may provide for an anonymous environment to learn more about homosexuality and having sex with other men, which may be especially appealing to YGBM at the beginning of their sexual 
trajectory. Third, the Internet allows for the tailoring of specific preventive messages to sub-groups of YGBM. For example, preventive messages can be delivered to YGBM who indicate being involved in steady relationship or for YGBM who report multiple sex partners. However, there are also some disadvantages of the Internet. One disadvantage of the Internet is that not all YGBM are active online and as such an intervention delivered solely online may not reach the whole YGBM community. Also, it may prove to be difficult to attract YGBM to sites containing health promotion activities. For one, safe sex might be of limited concern for YGBM searching for information about homosexuality in general. Moreover, online interventions usually require active participation of intended participants and it should be first determined whether YGBM at the beginning of their sexual career are already willing to actively participate in such online interventions. If the Internet is predominantly used to search for same-sex partners, it is questionable whether YGBM searching for sex online are willing to take part in an online HIV-prevention intervention. Thus, it is important when planning the intervention that care must be taken for the selection the optimal setting for delivering the intervention. Above all, this decision must be based on the behavior identified in step one, and on evidence that the change objectives and the associated behavioral change methods are suitable for the specific setting. It may prove to be necessary to use multiple settings to deliver the intervention.

To sum up, in this section suggestions for intervention development and delivery have been provided. Most importantly, the determinant configuration provided in Table 7.1 provides information about which determinants the intervention should target. Moreover, the setting of the intervention must be carefully selected and although it seems that the Internet is a suitable environment, there are some limitations. These limitations suggest that other settings (i.e. offline settings) may also qualify as a setting for delivering the intervention. Most importantly, the decision where to deliver the intervention (or multiple interventions) must be based on the change objectives and the behavioral change techniques identified in step three. 


\section{Concluding remarks}

The studies described in the present thesis were part of Outcomes, an online longitudinal study on coming-out and sexual risk behavior of YGBM. This research project is unique in four ways. First, the Outcomes study is one of the first studies investigating sexual risk behavior of YGBM who are in the midst of their coming-out. Second, most research on sexual risk behavior of YGBM is cross-sectional, and of the few longitudinal studies that are described in the literature, most studies consists of only two data waves. In the Outcomes study, respondents are required to complete six biannual questionnaires. This enabled us to examine both cross-sectional and longitudinal patterns in sexual risk behaviors, which yielded unique insights into sexual risk behaviors of YGBM. Finally, although a rather strict inclusion criteria was employed (i.e. first disclosure of sexual orientation in the year preceding the study), we still managed to attract 185 respondents. Moreover, drop-out rate was limited with $76 \%$ of respondents completing four questionnaires.

In sum, the research described in this dissertation provided insight into sexual risk behaviors and mental health concerns of YGBM in the Netherlands. The results of these studies support the need for the development of HIV preventive interventions targeting YGBM who are at the beginning of their sexual trajectory. In addition, the results warrants for the development of interventions to prevent mental health concerns among YGBM. Finally, because the determinant structure of sexual risk behavior of YGBM might change over time, we suggest that YGBM who are at the beginning of their sexual trajectory must be monitored continuously. Because of the continuous nature of the coming-out process, an open cohort seems to be an adequate method for the monitoring of the sexual behavior of YGBM in their comingout. The promising outcomes of the current project indicate that such an approach would indeed be feasible. 


\section{References}

1. UNAIDS, AIDS epidemic update: December 2007. 2007, UNAIDS: Genève.

2. Meyer, I.H., Prejudice, social stress, and mental health in lesbian, gay, and bisexual populations: Conceptual issues and research evidence. Psychological Bulletin, 2003. 129: 374-697.

3. Gras, L., A. van Sighem, C. Smit, S. Zaheri, H. Schuitemaker, and F. de Wolf, Monitoring of Human Immunodefienciy Virus (HIV) in The Netherlands 2007. 2007, Stichting HIV

Monitoring: Amsterdam.

4. Centre for Disease Control, HIVIAIDS and young men who have sex with men. 2009, US Department of Health and Human Services: Atlanta.

5. Hospers, H.J., T.T. Dörfler, and W. Zuilhof, Schorer Monitor 2007. 2007, Schorer: Amsterdam.

6. Hospers, H.J., T.T. Dörfler, and W. Zuilhof, Schorer Monitor 2008. 2008, Schorer: Amsterdam.

7. Public Health Agency of Canada, HIV and AIDS in Canada. Surveillance Report to June 30, 2006. 2006, Surveillance and Risk Assessment Division, Centre for Infectious Disease Prevention and Control, Public Health Agency of Canada.

8. The UK Collaborative Group for HIV and STI Surveillance, Testing Times. HIV and other Sexually Transmitted Infections in the United Kingdom: 2007. 2007, Health Protection Agency, Centre for Infections: London.

9. Imrie, J. and A. Falkland, Annual report of trends and behaviour 2007. 2007, National Centre in HIV Social Research: Sydney, Australia.

10. Schindhelm, R.K. and H.J. Hospers, Sex with men before coming-out: relation to sexual activity and sexual risk-taking behaviour. Archives of Sexual Behavior, 2004. 33: 585-591. 
11. Troiden, R.R., Becoming homosexual: a model of gay identity acquisition Psychiatry, 1979. 42: 362-373.

12. Cass, V.C., Homosexual identity formation: testing a theoretical model. Journal of Sex Research, 1984. 22: 143-167.

13. Dube, E.M., The role of sexual behavior in the identification process of gay and bisexual males. The Journal of Sex Research, 2000. 37: 123-132.

14. Horrowitz, J.L. and M.D. Newcomb, A multidimensional approach to homosexual identity. Journal of Homosexuality, 2001. 42: 1-19.

15. Maguen, S., F.J. Floyd, R. Bakeman, and L. Armistead, Developmental milestones and disclosure of sexual orientation among gay, lesbian, and bisexual youths. Applied Developmental Psychology, 2002. 23: 219-233.

16. Savin Williams, R.C., A critique of research on sexual-minority youths. Journal of Adolescence, 2001. 24: 5-13.

17. Rosario, M., E.W. Schrimshaw, and J. Hunter, Predicting different patterns of sexual identity development over time among Lesbian, Gay, and Bisexual youths: a cluster analytic approach. American Journal of Community Psychology, 2008. 42: 266-282.

18. Elisason, M.J., Identity formation for lesbian, bisexual, and gay persons: beyond a "minoritizing" view. Journal of Homosexuality, 1996. 30: 31-58.

19. Savin-Williams, R.C. and L.M. Diamond, Sexual identity trajectories among sexual-minority youths: gender comparisons. Archives of Sexual Behavior, 2000. 29: 607-627.

20. Rosario, M., E.W. Schrimshaw, and J. Hunter, A model of sexual risk behaviors among young gay and bisexual men: longitudinal associations of mental health, substance abuse, sexual abuse, and the coming-out process. AIDS Education and Prevention, 2006. 15: 444-460.

21. Friedman, M.S., M.P. Marshal, R. Stall, J. Cheong, and E.R. Wright, Gay-related development, early abuse and adult health outcomes among gay males. AIDS and Behavior, 2008. 12: 891-902.

22. Bartholomew, K., G.S. Parcel, G. Kok, and N. Gottlieb, Planning health promotion programs. An intervention mapping approach. 2006, San Franscisco: Jossey-Bass.

23. Hospers, H.J., T.T. Dörfler, and W. Zuilhof, Schorer Monitor 2006. 2006, Schorer: Amsterdam. 
24. Fishbein, M. and I. Ajzen, Belief, attitude, intention and behavior. An introduction to theory and research. 1975, Reading, MA: Addison-Wesley.

25. Azjen, I., The theory of planned behavior. Organizational Behavior and Human Decision Processes, 1991. 50: 197-211.

26. Fishbein, M. and I. Ajzen, Predicting and changing behavior. The reasoned action approach. 2009, New York, NY: Taylor \& Francis Group.

27. Bandura, A., Self-efficacy: the exercise of control. 1997, New York: Freeman.

28. Fergusson, D.M., L.J. Horwood, E.M. Ridder, and A.L. Beautrais, Sexual orientation and mental health in a birth cohort of young adults. Psychological Medicine, 2005. 35: 971981.

29. Cochran, S.D., J.G. Sullivan, and V.M. Mays, Prevalence of mental disorders, psychological distress, and mental services use among lesbian, gay, and bisexual adults in the United States. Journal of Consulting and Clinical Psychology, 2003. 71: 53-61.

30. De Graaf, R., T. Sandfort, and M. Ten Have, Suicidality and sexual orientation: differences between men and women in a general population-based sample from the Netherlands. Archives of Sexual Behavior, 2006. 35: 253-262.

31. Rosario, M., E.W. Schrimshaw, and J. Hunter, Predictors of substance use over time among gay, lesbian, and bisexual youths: An examination of three hypotheses. Addictive Behaviors, 2004. 29: 1623-1631.

32. Rosario, M., J. Hunter, S. Maguen, M. Gwadz, and R. Smith, The coming-out process and its adaptational and health-related associations among gay, lesbian, and bisexual youths: stipulation and exploration of a model. American Journal of Community Psychology, 2001. 29: 133-160.

33. Franssens, D. and H.J. Hospers, Outcomes. Deelrapport 2. 2009, Maastricht University: Maastricht.

34. Franssens, D., M. Koning, and H.J. Hospers, Outcomes. Deelrapport 1. 2007, Maastricht University: Maastricht.

35. Bolding, G., M. Davis, G. Hart, L. Sherr, and J. Elford, Where young MSM meet their first sexual partner: the role of the Internet. AIDS and Behavior, 2007. 11: 522-526.

36. McFarlane, M., S.S. Bull, and C.A. Rietmeijer, Young adults on the internet: Risk behaviors for sexually transmitted diseases and HIV. Journal of Adolescent Health, 2002. 31: 11-16. 
37. McFarlane, M., S.S. Bull, and C.A. Reitmeijer, The Internet as a newly emerging risk environment for sexually transmitted diseases. JAMA: Journal of the American Medical Association, 2000. 284: 443-446.

38. Garofalo, R., A. Herrick, B.S. Mustanski, and G.R. Donenberg, Tip of the iceberg: Young men who have sex with men, the Internet, and HIV risk. American Journal of Public Health, 2007. 97: 1113-1117.

39. Balthasar, H., A. Jeannin, and F. Dubois-Arber, First anal intercourse and condom use among men who have sex with men in Switzerland. Archives of Sexual Behavior, In press.

40. Horvath, K.J., S. Rosser, and G. Remafedi, Sexual risk taking among young internet-using men who have sex with men. American Journal of Public Health, 2008. 98: 1059-1067.

41. Hospers, H.J., G. Kok, P. Harterink, and O. de Zwart, A new meeting place: chatting on the internet, $e$-dating and sexual risk behaviour among Dutch men who have sex with men. AIDS, 2005. 19: 1097-1101.

42. Davidovich, U., J. de Wit, and W. Stroebe, Assessing sexual risk behaviour of young gay men in primary relationships: the incorporation of negotiated safety and negotiated safety compliance. AIDS, 2000. 14: 701-706.

43. Hospers, H.J., Homosexual men and the HIV epidemic. Understanding and changing risk behavior. 1999, Maastricht University: Maastricht.

44. Elford, J., G. Bolding, and L. Sherr, Seeking sex on the internet and sexual risk behaviour among gay men using London gyms. AIDS, 2001. 15: 1409-1415.

45. CDC, HIV/AIDS surveillance report, 2005. 2005, U.S. Department of Health and Human Services, Centre for Disease Control and Prevention: Atlanta.

46. Dudley, M.G., S.S. Rostosky, B.A. Korfhage, and R.S. Zimmerman, Correlates of high-risk sexual behavior among young men who have sex with men. AIDS Education and Prevention, 2004. 16: 328-340.

47. Hall, H.I., R.H. Byers, Q. Ling, and L. Espinoza, Racial/Ethnic and age disparities in HIV prevalence and disease progression among men who sex with men in the United States. American Journal of Public Health, 2007. 97: 1060-1066. 
48. Hogg, R.S., A.E. Weber, K. Chan, S.L. Martindale, D. Cook, M.L. Miller, et al., Increasing incidence of HIV infections among younger gay and bisexual men in Vancouver. AIDS, 2001. 15: 1321-1322.

49. Koblin, B.A., L.V. Torian, V. Guilin, L. Ren, D.A. MacKellar, and L.A. Valleroy, High prevalence of HIV infection among young men who have sex with men in New York City. AIDS, 2000. 14: 1793-1800.

50. Lightfoot, M., J. Song, M.J. Rotheram-Borus, and P. Newman, The influence of partner type and risk status on the sexual behavior of young men who have sex with men living with $H I V / A I D S$. Journal of Acquired Immune Deficiency Syndromes, 2005. 38: 61-68.

51. Valleroy, L.A., D.A. MacKellar, J.M. Karon, D.H. Rosen, W. McFarland, D.A. Shehan, et al., HIV prevalence and associated risks in young men who have sex with men. JAMA: Journal of the American Medical Association, 2000. 284: 198-204.

52. Webster, R.D., W.W. Darrow, J.P. Paul, R.A. Roark, W.J. Woods, and R.R. Stempel, HIV infection and associated risk among young men who have sex with men in a Florida resort community. Journal of Acquired Immune Deficiency Syndromes, 2003. 33: 223-231.

53. Warren, J.C., M.I. Fernandez, G.W. Harper, M.A. Hidalgo, O.B. Jamil, and R.S. Torres, Predictors of unprotected sex among young sexually active African American, Hispanic, and White MSM: the importance of ethnicity and culture. AIDS and Behavior, 2008. 12: 459-468.

54. Rangel, M.C., L. Gavin, C. Reed, M.G. Fowler, and L.M. Lee, Epidemiology of HIV and AIDS among adolescents and young adults in the United States. Journal of Adolescent Health, 2006. 39: 156-163.

55. Weber, A.E., K. Chan, C. George, R.S. Hogg, R.S. Remis, S. Martindale, et al., Risk factors associated with HIV infection among young gay and bisexual men in Canada. Journal of Acquired Immune Deficiency Syndrome, 2001. 28: 81-88.

56. Johnston, S., J.B.F. de Wit, M. Janssen, R.A. Coutinho, and G.J.P. van Griensven, Do today's young homosexual men practice safer sex than today's older homosexual men did when they were young? An analysis of sexual behavior change across cohorts in Amsterdam. AIDS and Behavior, 1999. 3: 75-81.

57. Hospers, H.J., T.T. Dörfler, and W. Zuilhof, Monitoronderzoek 2003. 2003, Schorer: Amsterdam. 
58. CDC, HIV/STD risks in young men who have sex with men who do not disclose their sexual orientation--six U.S. cities, 1994-2000. 2003, CDC.

59. Xiridou, M., R. Geskus, J. de Wit, R. Coutinho, and M. Kretzschmar, The contribution of steady and casual partnerships to the incidence of HIV infection among homosexual men in Amsterdam. AIDS, 2003. 17: 1029-1038.

60. Prestage, G., P.V. de Ven, L. Mao, A. Grulich, S. Kippax, and J. Kaldor, Contexts for last occasions of unprotected anal intercourse among HIV-negative gay men in Sydney: The Health in Men cohort. AIDS Care, 2005. 17: 23-32.

61. Mao, L., J. Crawford, P. Van De Ven, G. Prestage, A. Grulich, J. Kaldor, et al., Differences between men who report frequent, occasional or no unprotected anal intercourse with casual partners among a cohort of HIV-seronegative gay men in Sydney, Australia. AIDS Care, 2006. 18: 942-951.

62. MacKellar, D.A., L.A. Valleroy, G.M. Secura, B.N. Bartholow, W. McFarland, D. Shehan, et al., Repeat HIV testing, risk behaviors, and HIV seroconversion among young men who have sex with men: a call to monitor and improve the practice of prevention. Journal of Acquired Immune Deficiency Syndromes, 2002. 29: 76-85.

63. MacKellar, D.A., L.A. Valleroy, G.M. Secura, S. Behel, T. Bingham, D.D. Celentano, et al., Unrecognized HIV infection, risk behaviors and perceptions of risk among young men who have sex with men. Journal of Acquired Immune Deficiency Syndrome, 2005. 38: 603-614.

64. Gorbach, P.M., L.N. Drumright, E.S. Daar, and S.J. Little, Transmission behaviors of recently HIV-infected men who have sex with men. Journal of Acquired Immune Deficiency Syndrome, 2006. 42: 80-85.

65. De Graaf, H., S. Meijer, J. Poelman, and I. Vanwesenbeeck, Seks onder je 25ste. Seksuele gezondheid van Jongeren in Nederland anno 2005. 2005, Rutgers Nisso Groep/ SOA AIDS Nederland: Delft.

66. Hospers, H.J., P. Harterink, K. Van den Hoek, and J. Veenstra, Chatters on the internet: a special target group for HIV prevention. AIDS CARE, 2002. 14: 539-544. 
67. Schwarcz, S., S. Scheer, W. McFarland, M. Katz, L. Valleroy, S.Y. Chen, et al., Prevalence of HIV-infection and predictors of $H I V$-transmission sexual risk behaviors among men who have sex with men. American Journal of Public Health, 2007. 97: 1067-1075.

68. Léobon, A. and L.R. Frigault, Frequent and systematic unprotected anal intercourse among men using the Internet to meet other men for sexual purposes in France: results from the "Gay Net Barometer 2006 " survey. AIDS Care, 2008. 20: 478484.

69. Osmond, D.H., L.M. Pollack, J.P. Paul, and J.A. Catania, Changes in prevalence of HIV infection and sexual risk behavior in men who have sex with men in San Francisco: 1997-2002. American Journal of Public Health, 2007. 97: 1677-1683.

70. Štulhofer, A., V. Baćak, I. Božičević, and J. Begovac, HIVrelated sexual risk taking among HIV-negative men who have sex with men in Zagreb, Croatia. AIDS and Behavior, 2008. 12: 505-512.

71. Crawford, J.M., P. Rodden, S. Kippax, and P. Van de Ven, Negotiated safety and other agreements between men in relationships: Risk practice redefined. International Journal of STD and AIDS, 2001. 12: 164-170.

72. Kippax, S., J. Noble, G. Prestage, J.M. Crawford, and D. Campbell, Sexual negotiation in the AIDS era: Negotiated safety revisited. AIDS, 1997. 11: 191-197.

73. U.S. Census Bureau, Census 2008. Demographic profile, Table DP-1. 2008.

74. Statistics Netherlands, Population by origin and generation. 2008.

75. Gibbons, F.X., M. Gerrard, H. Blanton, and D.W. Russell, Reasoned action and social reaction: willingness and intention as independent predictors of health risk. Journal of Personality and Social Psychology, 1998. 74: 1164-1180.

76. Van Empelen, P. and G. Kok, Condom use in steady and casual sexual relationships: planning, preparation and willingness to take risks among adolescents. Psychology and health, 2006. 21: 165-181.

77. Keuzenkamp, S. and D. Bos, Out in the Netherlands. Acceptance of homosexuality in the Netherlands. 2007, The Netherlands Institute for Social Research/scp: The Hague. 
78. Greenwood, G., M.V. Relf, B. Huang, L.M. Pollack, J.A. Canchola, and J.A. Catania, Battering victimization among a probability-based sample of men who have sex with men. American Journal of Public Health, 2002. 92: 1964-1969.

79. Koblin, B.A., L. Torian, G. Xu, V. Guilin, H. Makki, D. MacKellar, et al., Violence and HIV-related risk among young men who have sex with men. AIDS Care, 2006. 18: 961-967.

80. Kok, G., P. Harterink, P. Vriens, O. De Zwart, and H.J. Hospers, The Gay cruise: developing theory-and evidencebased Internet HIV-prevention. Sexuality Research and Social Policy, 2006. 3: 52-67.

81. Mikolajczak, J., G. Kok, and H.J. Hospers, Queermasters: developing a theory-and evidence-based Internet HIVprevention intervention to promote HIV-testing among men who have sex with men. Applied Psychology: An International Review, 2008. 54: 681-697.

82. Elford, J., G. Bolding, and L. Sherr, High-risk sexual behaviour increases among London gay men between 1998 and 2001: What is the role of HIV optimism? AIDS, 2002. 16: 15371544.

83. Van de Ven, P., P. Rawstorne, J. Crawford, and S. Kippax, Increasing proportions of Australian gay and homosexually active men engage in unprotected anal intercourse with regular and with casual partners. AIDS CARE, 2002. 14: 335-341.

84. Xia, Q., D.H. Osmond, M. Tholandi, L.M. Pollack, W. Zhou, J.D. Ruiz, et al., HIV Prevalence and Sexual Risk Behaviors Among Men Who Have Sex With Men: Results From a Statewide Population-Based Survey in California. Journal of Acquired Immune Deficiency Syndromes, 2006. 41: 238-245.

85. Franssens, D., H.J. Hospers, and G. Kok, Risky debut: high levels of unprotected sex among young gay and bisexual men during their coming-out in the Netherlands. Manuscript submitted for publication, 2009.

86. Kok, G., H. Schaalma, R.A.C. Ruiter, P. Van Empelen, and J. Brug, Intervention Mapping: a protocol for applying health psychology theory to prevention programs. Health Psychology, 2004. 9: 85-98.

87. MacKellar, D.A., L.A. Valleroy, G.M. Secura, S. Behel, T. Bingham, D.D. Celentano, et al., Perceptions of lifetime risk and actual risk for acquiring HIV among young men who have sex with men. AIDS and Behavior, 2007. 11: 263-270. 
88. MacKellar, D.A., L.A. Valleroy, J.E. Anderson, S. Behel, G.M. Secura, T. Bingham, et al., Recent HIV testing among young men who have sex with men: correlates, contexts, and HIV seroconversion. Sexually Transmitted Diseases, 2006. 33: 183192.

89. Chen, S.Y., D. Weide, and W. McFarland, Are the recent increases in sexual risk behavior among older or younger men who have sex with men? Answer: both. AIDS, 2003. 17: 942943.

90. Salomon, E.A., M.J. Mimiaga, M.J. Husnik, S.L. Welles, M.A. Manseau, A.B. Montenegro, et al., Depressive symptoms, utilization of mental health care, substance use and sexual risk among young men who have sex with men in EXPLORE: implications for age-specific interventions. AIDS and Behavior, In press.

91. Huebner, D.M., G.M. Rebchook, and S.M. Kegeles, $A$ longitudinal study of the association between treatment optimism and sexual risk behavior in young adult gay and bisexual men. Journal of Acquired Immune Deficiency Syndrome, 2004. 37: 1514-1518.

92. Elford, J., G. Bolding, M. Maguire, and L. Sherr, Combination therapies for HIV and sexual risk behavior among gay men. Journal of Acquired Immune Deficiency Syndromes, 2000. 23: 266-271.

93. International Collaboration on HIV Optimism, HIV treatments optimism among gay men: an interpersonal perspective. Journal of Acquired Immune Deficiency Syndromes, 2003. 32: 454550.

94. Van de Ven, P., P. Rawstorne, T. Nakamura, J. Crawford, and S. Kippax, HIV treatments optimism is associated with unprotected anal intercourse with regular and with casual partners among Australian gay and homosexually active men. International Journal of STD and AIDS, 2002. 13: 181-183.

95. Albarracin, D., B.T. Johnson, M. Fishbein, and P.A. Muellerleile, Theories of reasoned action and planned behavior as models of condom use: A meta-analysis. Psychological Bulletin, 2001. 127(1): 142-161.

96. Franssens, D., H.J. Hospers, and G. Kok, Social cognitive determinants of condom use in a cohort of young gay and bisexual men. Aids Care, 2009. 21: 1471-1479. 
97 De Wit, J., T. Sandfort, E.M.M. De Vroome, F. Van Griensven, G. Kok, R. Coutinho, et al., Serial cross-sectional and longitudinal changes in sexual risk behaviour among homosexual men. Tijdschrift Sociale Gezondheidszorg, 1992. 70: 457-462.

98. Rawstorne, P., E. Digiusto, H. Worth, and I. Zablotska, Associations between crystal methamphetamine use and potentially unsafe sexual activity among gay men in Australia. Archives of Sexual Behavior, 2007. 36: 646-654.

99. Koblin, B.A., M.A. Chesney, M.J. Husnik, S. Bozeman, C. Celum, S. Buchbinder, et al., High-Risk behaviors among men who have sex with men in 6 US cities: Baseline data from the EXPLORE study. American Journal of Public Health, 2003. 93: 926-932.

100. Bartholow, B.N., S. Buchbinder, C. Celum, V. Goli, B.A. Koblin, M. Para, et al., HIV sexual risk behavior over 36 months of follow-up in the world's first HIV vaccine efficacy trail. Journal of Acquired Immune Deficiency Syndromes, 2005. 39: 90-101.

101. Koblin, B.A., M.J. Husnik, G. Colfax, Y. Huang, M. Madison, K.H. Mayer, et al., Risk factors for HIV infection among men who have sex with men. AIDS, 2006. 20: 731-739.

102. Brooks, R.A., S.J. Lee, P.A. Newman, and A.A. Leibowitz, Sexual risk behavior has decreased among men who have sex with men in Los Angeles but remains greater than that among heterosexual men and women. AIDS Education and Prevention, 2008. 20: 312-324.

103. Katz, M., W. McFarland, V. Guilin, M. Fenstersheib, M. Shaw, T. Kellogg, et al., Continuing high prevalence of HIV and risk behaviors among young men who have sex with men: the young men's survey in the San Francisco Bay Area in 1992 to 1993 and in 1994 to 1995. Journal of Acquired Immune Deficiency Syndromes, 1998. 19: 178-181.

104. Mao, L., J. Crawford, H.J. Hospers, G. Prestage, A. Grulich, J. Kaldor, et al., Serosorting in casual anal sex of HIV-negative gay men is noteworthy and is increasing in Sydney, Australia. AIDS, 2006. 20: 1204-1206.

105. Moreau-Gruet, F., F. Dubois-Arber, and A. Jeannin, Long-term HIVIAIDS-related prevention behaviours among men having sex with men: Switzerland 1992-2000. AIDS Care, 2006. 18: $35-43$. 
106. Kalichman, S.C., L. Eaton, D. White, C. Cherry, H. Pope, D. Cain, et al., Beliefs about treatments for HIVIAIDS and sexual risk behaviors among men who have sex with men, 1997-2006. Journal of Behavioral Medicine, 2007. 30: 497-503.

107. Ostrow, D., M.J. Silverberg, R.L. Cook, J.S. Chmiel, L. Johnson, X. Li, et al., Prospective study of attitudinal and relationship predictors of sexual risk in the Multicenter AIDS cohort study. AIDS and Behavior, 2008. 12: 127-138.

108. Kalichman, S.C., L. Eaton, D. Cain, C. Cherry, A. Fuhrel, M. Kaufman, et al., Changes in HIV treatment beliefs and sexual risk behaviors among gay and bisexual men, 1997-2005. Health Psychology, 2007. 26(5): 650-656.

109. Gosling, S.D., S. Varize, S. Srivastava, and O.P. John, Should we trust web-based studies? American Psychologist, 2004. 59: 93-104.

110. Rhodes, S.D., R.J. DiClimente, H. Cecil, K.G. Hergenrather, and L.J. Yee, Risk among men who have sex with men in the United States: a comparison of an internet sample and a conventional outreach sample. AIDS Education and Prevention, 2002. 14: 41-50.

111. Whittier, D.K., S. Seely, and J.S. St. Lawrence, A comparison of web-with paper-based surveys of gay and bisexual men who vacationed in a gay resort community. AIDS Education and Prevention, 2004. 16: 476-485.

112. Abraham, C., P. Sheeran, and M. Johnston, From health beliefs to self-regulation: theoretical advances in the psychology of action control. Psychology and Health, 1998. 13: 569-591.

113. Armitage, C.J. and M. Conner, The theory of planned behavior: assessment of predictive validity and 'perceived control'. British Journal of Psychology 1999. 38: 35-54.

114. Godin, G. and G. Kok, The theory of planned behavior: a review of its applications to health-related behaviours. American Journal of Health Promotion, 1996. 11: 87-98.

115. Kirby, D.B., B.A. Laris, and L.A. Rolleri, Sex and HIV education programs: their impact on sexual behaviors of young people throughout the world. Journal of Adolescent Health, 2007. 40: 206-217.

116. Rivis, A. and P. Sheeran, Descriptive norms as an additional predictor in the theory of planned behaviour: a meta-analysis. Current Psychology, 2003. 22: 218-233. 
117. Conner, M. and C.J. Armitage, Extending the theory of planned behavior: a review and avenues for further research. Journal of Applied Psychology, 1998. 28: 1430-1464.

118. Godin, G., M. Conner, and P. Sheeran, Bridging the intentionbehavior 'gap'. British Journal of Social Psychology, 2005. 44: 497-512.

119. Manstead, T. and D. Parker, Evaluating and extending the theory of planned behavior, in European Review of Social Psychology, W. Stroebe and M. Hewstone, Editors. 1995, Wiley: Chichester UK.

120. Sheeran, P., S. Orbell, and C. Abraham, Psychosocial correlates of heterosexual condom use: a meta-analysis. Psychological Bulletin 1999. 125: 90-132.

121. De Wit, J.B.F., W. Stroebe, E.M.M. De Vroome, T.G.M. Sandfort, and G.J.P. Van Griensven, Understanding AIDS preventive behavior with casual and primary partners in homosexual men: the theory of planned behavior and the information-motivation-behavioral-skills model. Psychology and Health, 2000. 15: 325-340.

122. Fisher, W.A., B.J. Rye, and J.D. Fisher, Understanding and promoting AIDS-preventive behavior: insights from the theory of reasoned action. Health Psychology, 1995. 14: 255-264.

123. Kok, G., H.J. Hospers, P. Harterink, and O. de Zwart, Socialcognitive determinants of HIV risk-taking intentions among men who date men through the Internet. AIDS Care, 2007. 19: 410-417.

124. Rye, B.J., W.A. Fisher, and J.D. Fisher, The theory of planned behavior and safer sexual behaviors of gay men. AIDS and Behavior, 2001. 5: 307-317.

125. Hogeweg, J.A. and H.J. Hospers, Monitoronderzoek 2000. 2000: Maastricht.

126. Rosario, M., K. Mahler, J. Hunter, and M. Gwadz, Understanding the unprotected sexual behaviors of gay, lesbian, and bisexual youths: An empirical test of the cognitiveenvironmental model. Health Psychology, 1999. 18: 272-280.

127. Conner, M. and P. Sparks, The theory of planned behavior and health behaviors, in Predicting health behavior: research and practice with social cognitive models, M. Conner and P. Norman, Editors. 2005, Open University Press: Maidenhead.

128. Albarracin, D., B.T. Johnson, and M.P. Zanna, The handbook of attitudes. 2005, New Jersey: Lawrence Erlbaum Associates. 
129. Schaalma, H.P. and G. Kok, A school IV-prevention program in the Netherlands, in Planning health promotion programs: An intervention mapping approach, L.K. Bartholomew, G.S. Parcel, G. Kok, and N.H. Gottlieb, Editors. 2006, Jossey-Bass: San Francisco. p. 511-544.

130. Udry, J.R. and K. Chantala, Risk Assessment of Adolescents with same-sex relationships. Journal of Adolescent Health, 2002. 31: 84-92.

131. Jorm, A.F., A.E. Korten, B. Rodgers, P.A. Jacomb, and H. Christensen, Sexual orientation and mental health: Results from a community survey of young and middle-aged adults. British Journal of Psychiatry, 2002. 180: 423-427.

132. Rosario, M., E.W. Schrimshaw, and J. Hunter, Psychological distress following suicidality among gay. lesbian, and bisexual youths: role of social relationships. Journal of Youth and Adolescence, 2005. 34: 149-161.

133. Rosario, M., E.W. Schrimshaw, J. Hunter, and M. Gwadz, Gay-related stress and emotional distress among gay, lesbian and bisexual youths: A longitudinal examination. Journal of Consulting and Clinical Psychology, 2002. 70: 967-975.

134. Kipke, M.D., K. Kubicek, G. Weiss, C. Wong, D. Lopez, E. Iverson, et al., The health and health behaviors of young men who have sex with men. Journal of Adolescent Health, 2007. 40: 342-350.

135. Safren, S.A. and R.G. Heimberg, Depression, hopelessness, suicidality, and related factors in sexual minority and heterosexual adolescents. Journal of Counseling and Clinical Psychology, 1999. 67: 859-866.

136. Silenzio, V.M.B., J.B. Pena, P.R. Dubberstein, J. Cerel, and K.L. Knox, Sexual orientation and risk factors for suicidal ideation and suicide attempts among adolescents and young adults. American Journal of Public Health, 2007. 97: 2017-2019.

137. Perdue, T., H. Hagan, H. Thiede, and L. Valleroy, Depression and HIV risk behavior among Seattle-area injection drug users and young men who have sex with men. AIDS Education and Prevention, 2003. 15: 81-92.

138. Mays, V.M. and S.D. Cochran, Mental health correlates of perceived discrimination among lesbian, gay, and bisexual adults in the United States. American Journal of Public Health, 2001. 91: 1869-1876. 
139. Halpin, S.A. and M.W. Allen, Changes on psychosocial wellbeing during stages of gay identity development. Journal of Homosexuality, 2004. 47: 109-126.

140. Ellemers, N. and M. Barreto, Social identity and selfpresentation at work: how attempts to hide a stigmatized identity affect emotional well-being, social inclusion and performance. Netherlands Journal of Psychology, 2006. 62: 5157.

141. Pachankis, J.E., The psychological implications of concealing a stigma: a cognitive-affective-behavioral model. Psychological Bulletin, 2007. 133: 328-345.

142. Rowen, C.J. and J.P. Malcolm, Correlates of internalized homophobia and homosexual identity formation in a sample of gay men. Journal of Homosexuality, 2002. 43: 77-92.

143. Frost, D.M. and I.H. Meyer, Internalized homophobia and relationship quality among lesbians, gay men, and bisexuals. Journal of Counseling Psychology, 2009. 56: 97-109.

144. Hershberger, S.L. and A.R. D'Augelli, The impact of victimization on the mental health and suicidality of lesbian, gay, and bisexual youths. Developmental Psychology, 1995. 31: 65-74.

145. Friedman, M.S., G.F. Koeske, A.J. Silvestre, W.S. Korr, and E.W. Sites, The impact of gender-role nonconforming behavior, bullying, and social support on suicidality among gay male youth. Journal of Adolescent Health, 2006. 38: 621-623.

146. Williams, T., J. Connolly, D. Pepler, and W. Craig, Peer victimization, social support, and psychosocial adjustment of sexual minority adolescents. Journal of Youth and Adolescence, 2005. 34: 471-482.

147. McLaren, S., B. Jude, and A.J. McLachlan, Sense of belonging to the general and gay communities as predictors of depression among Australian gay men. International Journal of Men's Health, 2008. 7: 90-99.

148. Derogatis, L.R. and N. Melisaratos, The brief symptom inventory: an introductory report. Psychological Medicine, 1983. 2: 595-605.

149. De Beurs, E. and F. Zitman, De Brief Symptom Inventory (BSI). De betrouwbaarheid en validiteit van een handzaam alternatief voor de SCL-90. Maandblad Geestelijke Volksgezondheid, 2006. 61: 120-141. 
150. Currie, M.R., E.G. Cunningham, and B.M. Findlay, The Short Internalized Homonegativity Scale: Examination of the Factorial Structure of a New Measure of Internalized Homophobia. Educational and Psychological Measurement, 2004. 64: 1053-1067.

151. Aiken, L.S. and S.G. West, Multiple regression: Testing and interpreting interactions. 1991, Newbury Park, CA: Sage.

152. Preacher, K.J., P.J. Curran, and D.J. Bauer, Computational tools for probing interactions in multiple linear regression, multilevel modelling, and latent curve analysis. Journal of Educational and Behavioral Statistics, 2006. 31: 437-448.

153. Cohen, J. and P. Cohen, Applied multiple regression/correlation analysis for the behavioral sciences. 2nd ed. 1983, Hillside, NJ: Erlbaum.

154. Herek, G.M., J.R. Gillis, and J.C. Cogan, Psychological sequelae of hate-crime victimization among lesbian, gay, and bisexual adults. Journal of Consulting and Clinical Psychology, 1999. 67: 945-951.

155. Mills, T.C., J. Paul, R. Stall, L. Pollack, J. Canchola, J.Y. Chang, et al., Distress and depression in men who have sex with men: the urban men's health study. American Journal of Psychiatry, 2004. 161: 278-285.

156. Rosario, M., E.W. Schrimshaw, and J. Hunter, Disclosure of sexual orientation and subsequent substance use and abuse among lesbian, gay, and bisexual youths: critical role of disclosure reactions. Psychology of Addictive Behaviors, 2009. 23: $175-184$.

157. Lewis, R.J., V.J. Derlega, E.G. Clarke, and J.C. Kuang, Stigma Consciousness, Social Constraints, and Lesbian Well-Being. Journal of Counseling Psychology, 2006. 53: 48-56.

158. Hatzenbuehler, M.L., K.A. McLaughlin, and S. NolenHoeksema, Emotion regulation and internalizing symptoms in a longitudinal study of sexual minority and heterosexual adolescents. The Journal of Child Psychology and Psychiatry, 2008. 49: 1270-1278.

159. Fergusson, D.M., L.J. Horwood, and A.L. Beautrais, Is sexual orientation related to mental health problems and suicidality in young people? Archives of General Psychiatry, 1999. 56: 876880 . 
160. King, M., J. Semlyen, S.S. Tai, H. Killaspy, D. Osborn, D. Popelyuk, et al., A systematic review of mental disorder, suicide, and deliberate self harm in lesbian, gay and bisexual people. BMC Psychiatry, 2008. 8: 70-87.

161. Sandfort, T.G.M., R. De Graaf, R.V. Bijl, and P. Schnabel, Same-sex sexual behavior and psychiatric disorders. Archives of General Psychiatry, 2001. 58: 85-90.

162. Franssens, D. and H.J. Hospers, Outcomes. Deelrapport 2. 2008, Maastricht University: Maastricht.

163. Bolding, G., M. Davis, G. Hart, L. Sherr, and J. Elford, Gay men who look for sex on the Internet: Is there more HIV/STI risk with online partners? AIDS, 2005. 19: 961-968.

164. Mercer, C.H., K. Wellings, W. MacDowell, S. McManus, K.A. Fenton, A.M. Johnson, et al., First sexual partnerships: age differences and their significance: empirical evidence from the 2000 British National Survey of Sexual Attitudes and LifeStyles ('Natsal 2000'). Journal of Adolescent Health, 2006. 39: 87-95.

165. Zablotska, I., G. Prestage, A. Frankland, J. Crawford, S. Kippax, R. Sutherland, et al., Sydney Gay Community Periodic Survey. February 1996 to August 2006. 2007, NCHSR: Sydney.

166. MacKellar, D.A., L.A. Valleroy, S. Behel, G.M. Secura, T. Bingham, D.D. Celentano, et al., Unintentional HIV exposures from young men who have sex with men who disclose being HIV-negative. AIDS, 2006. 20: 1637-1644.

167. Janssen, M., J. De Wit, W. Stroebe, and F. Van Griensven, Educational status and risk of HIV in young gay men. Journal of Health Psychology, 2000. 54: 487-499.

168. Galvan, F.H., R.L. Collins, D.E. Kanouse, P. Pantoja, and D. Golinelli, Religiosity, denominational affiliation, and sexual behaviors among people with HIV in the United States. Journal of Sex Research, 2007. 44: 49-58.

169. Davidovich, U., J. de Wit, and W. Stroebe, Relationship Characteristics and Risk of HIV Infection: Rusbult's Investment Model and Sexual Risk Behavior of Gay Men in Steady Relationships. Journal of Applied Social Psychology, 2006. 36: 20-40.

170. Crawford, J.M., S.C. Kippax, L. Mao, P. Van de Ven, G.P. Prestage, A.E. Grulich, et al., Number of risk acts by relationship status and partner serostatus: findings from the HIM cohort of homosexually active men in Sydney, Australia. AIDS and Behavior, 2006. 10: 325-331. 
171. Stolte, I.G., N.H.T.M. Dukers, R.B. Geskus, R.A. Coutinho, and J.B.F. De Wit, Homosexual men change to risky sex when perceiving less threat of HIVIAIDS since availability of highly active antiretroviral therapy: a longitudinal study. AIDS, 2004. 18: 303-309.

172. Janssen, M., J. De Wit, H.J. Hospers, and F. Van Griensven, Educational status and young Dutch gay men's beliefs about using condoms. AIDS Care, 2000. 13: 41-56.

173. Gold, R.S. and M.J. Skinner, Situational factors and thought processes associated with unprotected intercourse among young gay men. AIDS, 1992. 6: 1021-1030.

174. Folkman, S., M.A. Chesney, L. Pollack, and C. Phillips, Stress, coping, and high-risk sexual behavior. Health Psychology, 1992. 11: 218-222.

175. Crepaz, N. and G. Marks, Are negative affective states associated with HIV sexual risk behaviors? A meta-analytic review. Health Psychology, 2001. 20: 291-299. 



\section{Summary}

Men who have sex with men (MSM) continue to be at risk of acquiring HIV through unprotected anal intercourse (UAI). Research consistently shows that younger age is an important risk factor for sexual risk behaviour (i.e. UAI), and it has been suggested that young MSM are in part responsible for recent increases in HIV-infections. Therefore, preventive interventions are needed to encourage young MSM to always practice safe sex. A better understanding of the reasons why young MSM engage in sexual risk behaviour may guide the development of these preventive interventions. Next to sexual health, young MSM may face other health problems. There is ample evidence that YGBM are at increased risk of emotional distress (i.e. depressive feelings) compared to their heterosexual counterparts. This elevated risk of emotional distress has been attributed to the experience of a unique set of stressors specifically related to belonging to a sexual minority. The aim of the present thesis was to describe the results of Outcomes. Outcomes is an online cohort on coming-out and sexual behaviour of young gay and bisexual men (YGBM) who where - at study entry - in the midst of their coming-out. In Outcomes, participants completed 6 biannual questionnaires on topics such as coming-out, sexual behaviour and mental health. The main focus of the present thesis was on sexual behaviour and on determinants of sexual risk behaviour of YGBM. A secondary aim was to investigate correlates of mental health concerns of YGBM.

In order to get a good understanding of the rite of passage with respect to sexual behaviours of YGBM, in chapter 2 a study was described on condom use during sexual debut of YGBM. More specifically, three research questions pertaining to the sexual debut 
were addressed: 1) where do YGBM meet their first same-sex partner; 2) how many YGBM use a condom during their first episode of anal intercourse; and 3) does meeting the first same-sex partner online facilitate sexual risk behaviour? The results showed that many YGBM are very active online, with about half the participants visiting gay-sites on a daily basis. Furthermore, about half of the participants met their first same-sex partner with whom they had anal intercourse online. Forty-five percent of the participants did not use condoms during their first episode of anal intercourse and, in contrast to other studies, meeting the first same-sex partner online was not associated with more sexual risk behaviour. These findings call for specific HIV-prevention activities encouraging YGBM to use condoms during their sexual debut and these intervention should be delivered both offline and online.

The next step in understanding sexual behaviour of YGBM is to determine whether YGBM continue to practice unsafe sex after their sexual debut. Therefore, in chapter 3 , rates of unprotected anal intercourse in the six months preceding the first wave of Outcomes were explored for steady (i.e. someone with whom participants have a serious romantic relationship), regular (i.e. someone with whom participants regularly have sex with without being involved in a serious romantic relationship) and casual partners (i.e. someone with whom respondents never had sex with before). The results showed that many YGBM continue to have UAI after their sexual debut. Of the YGBM with a steady partner, $43 \%$ reported UAI with a steady partner in the preceding six months of the first wave of Outcomes. Of the YGBM with a regular partner, $33 \%$ reported UAI with regular partners and of the YGBM with a casual partner, 33\% reported UAI with casual partners in the preceding six months of the first wave of Outcomes. Furthermore, these rates of UAI were unrelated to demographic characteristics or to sexual history variables (such as age at first samesex experience, age first same-sex partner, or number of same-sex partners). Thus, after an unsafe sexual debut, a substantial number of YGBM continued to have unsafe sex with steady, regular or casual partners. These cross-sectional findings raised the question how these rates of UAI would develop over time. 
In order to explore cross-sectional sexual behaviour patterns in four waves of Outcomes, in chapter 4 sexual behaviour with steady, regular and casual partners were combined to form one behavioural index: no sex partners or no anal intercourse, protected anal intercourse, and unprotected anal intercourse. The results showed that on each of four data waves between $34 \%$ and $37 \%$ of the participants had unprotected sex, which is well in line with other Dutch MSM Monitoring studies. In order to explore longitudinal patterns of sexual behaviour, the cross-sectional behavioural index was further dichotomized into safe (i.e. no partners, or no sex, no anal intercourse, or protected anal intercourse) versus unsafe (i.e. unprotected anal intercourse). Based on this dichotomous index, there were 16 possible combinations over four waves $\left(2^{4}\right)$, which were classified into three risk groups: consistent no risk, consistent risk, and variable risk. The results showed that $14 \%$ of the participants consistently engaged in sexual risk behaviour (i.e. reported UAI at each of four data waves) and that $45 \%$ variably engaged in sexual risk behaviour (i.e. engaged in UAI at one or more data waves but not on all four data waves). Furthermore, the longitudinal analysis showed that reporting multiple same-sex sexpartners (i.e. $\geq 4$ ), ever being tested for HIV, being more optimistic about HIV, and having a lower behavioural intention to use condoms, are related to longitudinal sexual risk behaviour. In sum, crosssectional analysis of patterns of UAI showed that on each data wave about one third of the participants engaged in UAI. Moreover, $14 \%$ consistently engaged in UAI at each of four data waves, and $45 \%$ variably engaged in UAI.

The studies described in chapters 2, 3 and 4 provided insights into the sexual trajectory of YGBM during and after their coming-out. The results showed that a substantial proportion of YGBM in Outcomes engaged in sexual risk behaviour and that effective HIVinterventions are needed to inform this special target group about condom use. In order to design such interventions, the social-cognitive determinants of condom use have to be known. In chapter 5, the theory of planned behaviour (TPB) was used to explore these socialcognitive determinants of condom use. In short, the TPB states that 
behaviour is best predicted by behavioural intentions, and that people form these intentions based on their attitudes, subjective norms, and perceived behavioural control. The results showed that participants with a maximal intention were more likely to use condoms with casual partners compared to participants with a less than maximal intention. Moreover, intentions were best predicted by attitudes, perceived behavioural control, personal norms and descriptive norms. For each of these predictors, the relevant beliefs were identified (see Table 5.3) and these beliefs should be changed with an HIV-prevention intervention.

In chapter 6 , correlates of mental health problems, operationalized in this study as feelings of depression, were explored. This research was based on the minority-stress framework which states that next to general life stressors (i.e. stress from daily hassles) YGBM experience additional stressors related to belonging to a sexual minority contribute to mental health concerns. More specifically, both internal (i.e. self-label as gay or bisexual, self-acceptance of sexual identity, internalized homonegativity, and concealment of sexual identity) and external stressors (experienced discrimination) were examined. The results showed that YGBM who experienced discrimination because of their sexual orientation report more depressive feelings compared to YGBM who did not experience discrimination. Furthermore, YGBM who hold negative attitudes towards homosexuality and who do not feel connected with the gay scene report more depressive feelings. Other stressors, such as self-label of sexual identity (homo- or bisexual), self-acceptance of sexual identity, and concealment of sexual identity (level of disclosure) were not related to feelings of depression. These results underscore the need for the development of interventions to prevent anti-gay harassment and to stimulate the acceptance of sexual minorities.

In conclusion, the research described in this thesis provided insight into the sexual behaviours of YGBM in the Netherlands. The results underscore the need for the development of HIV-preventive interventions targeting YGBM who are at the beginning of their sexual trajectory. Moreover, these studies provided information with respect 
to the content of such interventions. The next step in the development of these interventions should be to identify theoretical models and practical strategies to accomplish the desired changes in the determinants of condom use and to design and implement the actual intervention program. In addition, interventions need to be developed to prevent mental health concerns among YGBM. Finally, because both the patterns and the determinant structure of sexual risk behaviour may change over time, YGBM who are at the beginning of their sexual trajectory must be monitored continuously. An open cohort seems to be an adequate method for the continuous monitoring of sexual behaviour of YGBM. The promising outcomes of the current research project indicate that such an approach would be indeed feasible. 



\section{Nederlandse samenvatting}

Mannen die seks hebben met mannen (MSM) blijven een risico lopen op het krijgen van een HIV infectie als gevolg van het hebben van onbeschermde anale seks. Onderzoek laat steevast zien dat een jonge leeftijd een belangrijke risicofactor is voor seksueel risicogedrag (dat wil zeggen onbeschermde anale seks), en het wordt gesuggereerd dat jonge MSM voor een gedeelte verantwoordelijk zijn voor de recent geobserveerde toenames in HIV infecties. Vandaar dat er preventieve interventies nodig zijn om jonge MSM aan te sporen om altijd veilige seks te hebben. Een beter inzicht in de redenen waarom jonge MSM seksueel risicogedrag vertonen kan het ontwikkelen van zulke interventies bevorderen. Jonge MSM worden, naast de seksuele gezondheid, ook met andere gezondheidsproblemen geconfronteerd. Er is veel bewijs dat jonge MSM een verhoogde kans hebben op het ervaren van emotionele stress (dat wil zeggen depressieve gevoelens) vergeleken met hun heteroseksuele leeftijdsgenoten. Dit verhoogde risico op emotionele stress wordt verklaard doordat jonge MSM een unieke set van stressoren ervaren die specifiek samenhangen met het behoren tot een seksuele minderheid. Het doel van dit proefschrift was het beschrijven van de resultaten van Outcomes. Outcomes is een online cohort onderzoek over coming-out en seksueel gedrag van jonge homo- en biseksuele jongens die - aan het begin van deze studie midden in hun coming-out zaten. Deelnemers aan Outcomes vulden 6 zesmaandelijkse vragenlijsten in over onderwerpen zoals coming-out, seksueel gedrag en geestelijke gezondheid. De primaire focus in dit proefschrift lag op het seksuele gedrag en op de determinanten van seksueel risicogedrag van jonge homo- en biseksuele jongens. Een 
secundaire focus lag op het onderzoeken van correlaten van geestelijke gezondheid van jonge homo- en biseksuele jongens.

Om een beter beeld te krijgen van de seksuele ontwikkeling van jonge homo- en biseksuele jongens, werd in hoofdstuk 2 een studie beschreven over condoomgebruik tijdens hun seksuele debuut. Drie onderzoeksvragen die specifiek betrekking hebben op het seksuele debuut werden aan de orde gesteld: (1) waar ontmoeten jonge homoen biseksuele jongens tegenwoordig hun eerste mannelijke sekspartner; 2) hoeveel jonge homo- en biseksuele jongens gebruiken een condoom tijdens de eerste keer anale seks; en 3) leidt het online ontmoeten van de eerste mannelijke seks partner tot meer seksueel risicogedrag met die partner? De resultaten laten zien dat veel jonge homo- en biseksuele jongens erg actief zijn online, waarbij ongeveer de helft van de deelnemers dagelijks websites voor homoseksuelen bezoeken. Bovendien heeft ongeveer de helft van de deelnemers zijn eerste mannelijke seks partner op Internet ontmoet. Vijfenveertig procent van de deelnemers heeft geen condooms gebruikt tijdens de eerste keer anale seks, en, in tegenstelling tot andere studies, bleek het op internet ontmoeten van de eerste mannelijke seks partner niet samen te hangen met meer seksueel risicogedrag. Deze resultaten vragen om specifieke HIV preventieve activiteiten die jonge homo- en biseksuele jongens moeten aansporen om condooms te gebruiken tijdens hun seksuele debuut en deze interventies moeten zowel offline als online worden uitgevoerd.

De volgende stap in het inzicht verkrijgen van in het seksueel gedrag van jonge homo- en biseksuele jongens is het vaststellen of jonge homo- en biseksuele jongens na hun seksuele debuut doorgaan met het hebben van onbeschermde seks. Daarom werden in hoofdstuk 3 percentages onbeschermde anale seks in de 6 maanden voorafgaand aan de eerste meting van Outcomes onderzocht voor vaste (dat wil zeggen een partner met wie je een serieuze romantische relatie hebt), regular (dat wil zeggen een partner met wie deelnemers regelmatig seks hebben zonder dat ze met hem een serieuze romantische relatie hebben) en losse partners (dat wil zeggen iemand met wie ze nog nooit seks hebben gehad). De resultaten lieten zien dat veel jonge homo- en 
biseksuele jongens na hun seksuele debuut doorgaan met het hebben van onbeschermde anale seks. Van de jonge homo- en biseksuele jongens met een vaste partner in de 6 maanden voorafgaand aan de eerste meting rapporteerde $43 \%$ onbeschermde anale seks met een vaste partner. Van de jonge homo- en biseksuele jongens met een regular partner rapporteerde $33 \%$ onbeschermde anale seks en van de jonge homo- en biseksuele jongens met een losse partner rapporteerde $33 \%$ onbeschermde anale seks in de 6 maanden voorafgaand aan de eerste meting van Outcomes. Bovendien bleek dat deze percentages van onbeschermde seks niet samenhangen met demografische kenmerken of met seksuele geschiedenis variabelen (zoals leeftijd seksueel debuut, leeftijd eerste mannelijke sekspartner of aantal mannelijke sekspartners). Dus, na een onveilig seksueel debuut blijven een substantieel aantal jonge homo- en biseksuele jongens onbeschermde anale seks hebben met vaste, regular of losse partners. De volgende vraag die beantwoord moest worden is hoe deze cross-sectionele percentages zich ontwikkelen over een langere tijdsperiode.

Om de cross-sectionele seksuele gedragspatronen in 4 metingen van Outcomes te bekijken, werd in hoofdstuk 4 het seksueel gedrag met vaste, regular en losse partners gecombineerd in één gedragsmaat: geen sekspartners of geen anale seks, beschermde anale seks, en onbeschermde anale seks. De resultaten lieten zien dat op elk van de 4 metingen tussen de $34 \%$ en $37 \%$ van de deelnemers onbeschermde anale seks had, een percentage dat goed overeenkomt met andere Nederlandse MSM monitoronderzoeken. Om longitudinale patronen van seksueel risicogedrag te onderzoeken werd de cross-sectionele gedragsmaat gedichotomiseerd in veilig (geen partner of geen seks of geen anale seks of beschermde anale seks) versus onveilig (onbeschermde anale seks). Op basis van deze gedichotomiseerde index zijn er 16 gedragscombinaties over 4 metingen $\left(2^{4}\right)$ mogelijk. Deze combinaties werden weer verder onderverdeeld in drie risicogroepen: consequent geen risico, consequent risico, en variabel risico. De resultaten lieten zien dat $14 \%$ van de deelnemers consequent seksueel risicogedrag vertoonden (dat wil zeggen, ze hadden onbeschermde anale seks op elk van de 4 metingen) en $45 \%$ variabel risicogedrag (dat 
wil zeggen, ze hadden onbeschermde anale seks op één of meerdere metingen maar niet elk van de 4 de metingen). Daarnaast liet de longitudinale analyse zien dat longitudinaal seksueel risicogedrag samenhangt met seks hebben gehad met meerdere mannelijke sekspartners (dat wil zeggen met meer dan 4), met ooit zijn getest op HIV, met optimistisch zijn over HIV, en met het hebben van een lagere intentie om condooms te gebruiken. Samenvattend, de cross-sectionele analyse van patronen van onbeschermde anale seks lieten zien dat op elke meting ongeveer een derde van de deelnemers onbeschermde anale seks rapporteerde. Bovendien bleek dat $14 \%$ van de deelnemers consequent en $43 \%$ variabel onbeschermde anale seks rapporteerde.

De studies die in hoofdstuk 2, 3 en 4 beschreven zijn hebben inzicht gegeven in de seksuele ontwikkeling van jonge homo- en biseksuele jongens gedurende en na hun coming-out. De resultaten lieten zien dat een substantieel deel van jonge homo- en biseksuele jongens in Outcomes seksueel risicogedrag vertoonde en dus dat effectieve HIV preventieve interventies nodig zijn om deze speciale doelgroep te informeren over condoomgebruik. Om zulke interventies te ontwikkelen moeten de sociaal-cognitieve determinanten van condoomgebruik bekend zijn. In hoofdstuk 5 werd de theorie van gepland gedrag gebruikt om de sociaal-cognitieve determinanten van condoomgebruik te onderzoeken. Kort gezegd, de theorie van gepland gedrag zegt dat gedrag het best voorspeld wordt door de intentie om dat gedrag uit te voeren, en dat mensen deze intentie baseren op hun attitude, hun subjectieve normen en hun waargenomen gedragscontrole. De resultaten lieten zien dat deelnemers met een maximale intentie vaker condooms gebruiken met een losse partner vergeleken met deelnemers met een minder dan maxi male intentie. Bovendien bleek dat deze intenties het best werden voorspeld door attitudes, waargenomen gedragscontrole, persoonlijke normen, en descriptieve normen. Voor elk van deze voorspellers zijn de relevante overtuigingen in kaart gebracht (zie Table 5.3) en deze overtuigingen zouden dus moeten worden veranderd door middel van een HIV preventieve interventie. 
In hoofdstuk 6 werden voorspellers van mentale gezondheid, in deze studie geoperationaliseerd als depressieve gevoelens, onderzocht. Dit onderzoek was gebaseerd op het minority-stress raamwerk dat stelt dat naast algemene stressoren (zoals stress door het dagelijks leven), jonge homo- en biseksuele jongens additionele stressoren ervaren als gevolg van het behoren tot een seksuele minderheid die bijdragen aan mentale gezondheid. Specifieker gezegd, zowel interne (i.e. zelf labeling als homo of biseksueel, zelfacceptatie van seksuele identiteit, geinternaliseerde homonegativiteit, en het verhullen van de seksuele identiteit) als externe (i.e. ervaren discriminatie) stressoren werden onderzocht. De resultaten lieten zien dat jonge homo- en biseksuele jongens die werden gediscrimineerd vanwege hun seksuele identiteit meer depressieve gevoelens rapporteerden vergeleken met jonge homoen biseksuele jongens die niet werden gediscrimineerd. Bovendien bleek dat jonge homo- en biseksuele jongens die negatieve attitudes hadden ten opzichte van homoseksualiteit en die zich niet verbonden voelden met de homoscene meer depressieve gevoelens rapporteerden. Andere stressoren zoals zelf labeling als homo of biseksueel, zelfacceptatie van seksuele identiteit, en het verhullen van de seksuele identiteit waren niet gerelateerd aan gevoelens van depressie. Deze resultaten benadrukken het belang van het ontwikkelen van interventies ter voorkoming van anti-homo geweld en het stimuleren de acceptatie van seksuele minderheden.

Samenvattend, het onderzoek beschreven in dit proefschrift heeft inzicht gegeven in het seksuele gedrag van jonge homo- en biseksuele jongens in Nederland. De resultaten benadrukken het belang van de ontwikkeling HIV preventieve interventies die zich speciaal richten op jonge homo- en biseksuele jongens aan het begin van hun seksuele ontwikkeling. De Outcomes studies heeft ook informatie opgeleverd over de inhoud van zulke interventies. De volgende stap in de ontwikkeling van deze interventies is het selecteren van theoretische modellen en praktische strategieën om de benodigde veranderingen in de determinanten van condoomgebruik te bereiken en het implementeren van deze interventies. Bovendien, interventies dienen te worden ontwikkeld die mentale gezondheidsproblemen bij jonge 
homo- en biseksuele jongens pogen te voorkomen. Tot slot, omdat zowel de patronen van seksueel risicogedrag en de determinanten structuur van seksueel risicogedrag kunnen veranderen in de tijd, moeten jonge homo- en biseksuele jongens die aan het begin staan van hun seksuele ontwikkeling continue gemonitord worden. Een open cohort lijkt een adequate methode te zijn voor zo'n continue monitoren van het seksueel gedrag van jonge homo- en biseksuele jongens. De bemoedigende resultaten van het huidige onderzoeksproject laten zien dat zo'n benadering haalbaar is. 


\section{Acknowledgements}

I would like to thank everybody who has contributed in some way to the realisation of this thesis. A special thanks to Harm Hospers and Gerjo Kok for all their help while conducting this research and for their excellent supervision. A second special thanks to all my respondents, my students-assistants, my interviewers, and my recruiters. Also, a special thanks to my family and my friends. A final special thanks to H.S. and C.A. 



\section{Curriculum Vitae}

Dirk Franssens was born on the $29^{\text {th }}$ of October 1976 in Oirsbeek, the Netherlands. In 1994 he obtained his Middelbaar Algemeen Voortgezet Onderwijs (MAVO) diploma, and in 1996 he received his Hoger Algemeen Voortgezet Onderwijs (HAVO) diploma. After studying International Management at the Hogeschool Utrecht for two years, he worked for two years both in Utrecht (Cadans UVI) and Maastricht (KPN). In september 2000, he started studying psychology at Maastricht University in Maastricht. He graduated and received his doctorandus degree (master's degree) in Cognitive Psychology, specifically in cognitive ergonomics, in 2004. In January 2005, he started working as a Ph.D candidate at the department of Health Education and Health Promotion, at the faculty of Health, Medicine and life Sciences (FHML). After one year, he decided to switch to the faculty of Psychology and Neuroscience to start working as a Ph.D. candidate on the research presented in this thesis. 